\section{English Index}

\section{Loránd-Levente Pálfi*}

A

A-chain 8 9, 11

Aarup, Hanne

interpretation

research and training 22163

vs translation 1473

Abercrombie, David

standard English 17218

abstracts 25 121, 125-127

conference 28 95-99

and textual silence 28 95-99

academic conflicts 25 23-44

academic language 28 7-9

academic writing 25 23-44

criticism in 25 23-44

Chinese $2524 \mathrm{f}$

Czech 2525

English 2524

German 2525

Malaysian 2525

Polish 2525

plagiarism 28 7, 13 (def), 39-56

Japanese university students $\mathbf{2 8}$

16-31

learners of English 28 11-31

reasons for $\mathbf{2 8} 12$

in Western academia 2543

accent

as social marker 17 216-218

acceptability 8 7-21

access structure 1764 (def)
Accessibility Hierarchy, The $\mathbf{8} 21$

Accounting Dictionary. English-

Spanish, Spanish-English, SpanishSpanish

review 38 205-216

accounting terminology 32149

UK- and US-English 32 149f

\section{ACOI 32 16ff}

features

discoursal $3223 \mathrm{f}$

lexico-grammatical 32 20-23

rhetorical $3223 \mathrm{f}$

socio-cognitive $3225 \mathrm{f}$

surface-level $3219 \mathrm{f}$

acquis communautaire $\mathbf{3 4} 296$

action 19 241-251

action rules 32101

action scenes

in novels and their translations $\mathbf{3 4}$

199-221

acts $\rightarrow$ legal acts; speech acts

Adam, J.H.

distribution 36201 (def)

additions

connective $\mathbf{3 2} 245$

culture-bound information 32

$245 f$

down-toning 32 245, 247

elaborating 32245,247

emphasizing 32 245, 247

with minimal impact $32246 \mathrm{f}$

new-information 32 245, 247

non-verbal information $32245 \mathrm{f}$

obvious-information 32245

with significant impact $32246 \mathrm{f}$

Adell, J.

new genres 2396

\footnotetext{
* Loránd-Levente Pálfi Centre for Lexicography Aarhus School of Business, University of Aarhus Fuglesangs Allé 4 DK-8210 Aarhus V llp@asb.dk
}

Hermes - Journal of Language and Communication Studies no 39-2007 
adequacy 1215

communicative $\mathbf{1 0} 48$

target text $\mathbf{1 0} 45$

Adject Theory 38196

adjectival modification 31 65-79

adjectives

Arabic

in LSP texts 37154

French

in LSP texts 37154

groups

discontinuous 17 56-62

lexical density 31 71f

relational

terminologically relevant $37153 \mathrm{f}$ adjuncts

the adjunct rule 1016

Adler, Nancy J.

intra-cultural negotiations 1513

administrative language 31 31-43

adverbials 38197

scenic 12204

adverbs

groups

discontinuous 17 56-62

manner 18227

advertising 23 93-110, 99 (def), 2431

active 23101

banner ad 23101 (def)

commercial vs non-commercial

2399

computer advertisements 2431 -

55

consumer 23100

images and HT linkers 23 107-

109

industrial 23 99f

on the Internet 23 93-110

passive 23101

prestige $2399 \mathrm{f}$

statement length 23104 (fig)

use of personal pronouns 23106

(fig)

use of punctuation marks 23107

(fig) use of verb tenses 23105 (fig)

the verbal message 23100

the visual message $\mathbf{2 3} 100$

Äquivalenz

approximative 1212

facultative 1212

totale 1212

see also equivalence

affixes 2514 (def)

vs combining forms 25 17-19

affordance $2427 \mathrm{f}$

Ahmad, U.K.

Malaysian academic writing 2525

AI 25 116ff

$\mathrm{Al}, \mathrm{B}$

bilingual lexicography 8 101, 105f

collocations in dictionaries 8107

Albert, L.M.

code-switching 1546

Alcoba, Santiago

verbs 6187

Alexieva, Bistra

communication, negotiation and

interpreting 22165

interpretation

deletion 17277

methodology 22164

Algeo, J.

British and American differences

13120

Almlöv, Cecilia

institutional discourse

gender differences in $\mathbf{3 6} 135$

Alrø, Helle

dialogues 3845

Altaic language family $3559 f$

Altenberg, B

tone units 13 126-129

Amant, Kirk St.

knowledge mediation 377

American, business letters 3753

American Heritage Dictionary of

Science 
biotechnological terms in monolingual dictionaries 18103 107

anaphors 17280 (def)

Andersen, Anja

apologies 38 86, 97-103

Andersen, Henning drift 38192

linguistic change 38191

markedness $\mathbf{3 8} 190$

Andersen, Øivin

LSP research 19 251-261

Andersen, Poul H.

business rhetorics 278

Anderson, Joseph

proprioception $34204 \mathrm{f}$

Anderson, R.B.W.

interpreting 20211

Angelo, Thomas A.

teaching grammar $\mathbf{3 1} 98$

anglicisms

in French 22 198-203

Anglo-Germanic

concepts of politeness and time 15 53-76

vs Latin (=Hispanic/French etc.) cultures 15 54ff

$\mathrm{ANN} \rightarrow$ artificial neural networks anthropology

French 38 107f

vs American 38 107f

and language 38 107-109

modern conception of culture $\mathbf{3 8}$ 107

anti-essentialist exhortation 31198

antipassive 19 77-79

backgrounding $1984 \mathrm{f}$

foregrounding $1984 \mathrm{f}$

French $1979 f$

reflexive verbs 19 75-90

and reflexive $1980 \mathrm{f}$

AP

the internal structure of $\mathbf{1 0} 20 \mathrm{f}$

apologetic ethics 38 85-103

apologia 3891 (def) apologies 21 72-75, 38 85-103, 88

(def), 89 (def)

complicating circumstances $3896 \mathrm{f}$

as crisis response strategies $\mathbf{3 8}$

87-89

definition 38 86f

direct 2181

ideal ethical standard $3893 \mathrm{f}$

as image restoration strategies $\mathbf{3 8}$

87-89

indirect 21 74f, 81

manner of communication 3893 -

95

the process of apologizing 38 89-91

strategies $\mathbf{2 1} 74$

typology $\mathbf{3 8} 86 \mathrm{f}$

Arabic

adjectives

in LSP texts 37154

Arai, Kiwa

interpreting 14135

Western literature on 14138

arbitral tribunal 3286 (def)

composition

in the Malaysian Arbitration

Act $3288 \mathrm{f}$

arbitration 32 50f, 77ff, 86 (def)

commercial

international 32 7-11

Model Law 32 77-97

Malaysian

content and language 32 77-97

structure 32 81-85

arbitration agreement 3287 (def)

in the Malaysian Arbitration Act

3287

arbitration law 3272

across legal systems 32 13-33

international $3215 \mathrm{ff}$

regulative acts in 32 101-108

regulative strategies 32 99-114

Argument-chain $\rightarrow$ A-chain

argumentation

the backing 2718 
conflict and consensus in 15117 -

129

metacomments

person-oriented 15138

the qualifier 2718

the rebuttal 2718

strategies 27 17-23, 18 (fig)

in French company brochures

19 297-301

arguments

across national/cultural groups $\mathbf{1 5}$

125 (fig)

ad hominem 15 120f, 124, 126,

129, 139

ad rem 15 120f, 139

immediate 15 121f, 124, 126

mediate 15 121f, 126

price-related (in negotiations)

vs peripheral $15127 \mathrm{f}$

push- 15 122f, 139

yield- 15 122-124, 127, 139

arguments (of verbs) 38197

fundamental 38198

Argyris, C.

performance measurements 2710

Aristotle

ethics 3892

Great Chain of Being 30184

logic 570

metaphors 24 111-113, 113 (def)

persuasion 3749

rhetorics 3748 (def)

Arndt, $\mathrm{H}$.

Intergrammar 12 141-156

Arnold, J.V.

combining forms $2516 \mathrm{f}$

Arntz, Rainer

Roman heritage in German legal

language $\mathbf{3 1} 220$

articles

indefinite

and modality 31 234-236

artificial intelligence $25116 \mathrm{ff}$

artificial neural networks 6 32-42 representation and learning in $\mathbf{6}$

34-42

Askehave, Inger

genre analysis 239

medical texts 258

Asmuß, Birte

public services $\mathbf{3 8} 8$

aspect 7 166, 17 94f, 19 241-251

English 1476

vs Danish 10 46f

progressive 1046,48

Danish 1476

assertiveness 12 151ff

interpersonal

strategies for modulating 12 151f

assignment of benefits $990 \mathrm{f}$

Asturian

clitics in 19 98, 103

Atkins, Bowman

courtroom language 36 132ff

Atkins, B.T. Sue

dictionaries

electronic vs paper $3416 \mathrm{f}$

Atkinson, $\mathrm{P}$.

in-group language $\mathbf{2 8} 79$

Auchlin, Antoine

Chinese and French 13 351-353

Austin, J.L.

speech act theory 948

speech acts 672

utterances 949

auxiliaries 13 71-84, 19 101f

English vs Danish 7 164f

modal 19106

Spanish 31 231-234

Axelsen, Jens

Dansk-engelsk ordbog 7 117-121

axiological components 30 189f

axiological evaluation 30 179-196

concept of hierarchy $\mathbf{3 0} 184 \mathrm{f}$

sociocultural context $\mathbf{3 0} 185 \mathrm{f}$

axiological parameter

Faber and Mairal 30 182-186

axiological scales 30 186-189 
B

Baakes, K.

the technical writer 2367

Bache, Carl

English sentence analysis 12 186-

189

grammatical categories 19 241-251

Bachman, Lyle F.

translation 2617 quality assessment $\mathbf{2 6} \mathbf{1 8 f}$

Back Propagation 641

back-channeling 3 258-260

Bailey, Ch.-J.N.

fashionable English 17224

standard English 17218

Bakhtin, M.

primary vs secondary genres $\mathbf{2 3} 95$

BAL 12130

Ballard, M.

translation 2666

Balsgart, Karin

use of LSP dictionaries 10123

Baltic languages 3556

Baltic-Finnic languages 3558

Bank of English 7 71f

Bank, John

quality 16208 (def)

banner ad 23101 (def)

Bannert, Robert

prosody 3323

Bantu languages

electronic dictionaries 34 20-32

bar-notation 1012

bargain 1161 (def)

bargaining sequence 1194

see also negotiations

Barik, Henri C.

interpretation

errors and omissions 2253

simultaneous $\mathbf{1 0} 45$

Barsalou, L.W.

frame $\mathbf{3 4} 206$

Perceptual Symbol Systems $34202 \mathrm{f}$
Basedow, Jürgen

legal language 3616

baseline $13205 \mathrm{ff}$

values $13208 \mathrm{f}$

Basiswoordeboek van Afrikaans vs Nuwe Woordeboek sonder grense 38 217-220

Basque 3558

Bauer, Laurie neo-classical compunds 2515

Bazerman, C. genre analysis $\mathbf{1 6} 42$

Bazerman, Max H. negotiation 159 (def)

BBC English Dictionary review 13 353-356

$\mathrm{BBC}$ television exerting influence on young speech $\mathbf{3 8} 180$

BBC-English 17 217ff, 38183

BBI Combinatory Dictionary of

English

review 7 129-137

Beamer, Linda

communication expectations 3762

Beaudoin, Louis

legal translation in Canada $31221 \mathrm{f}$

Becerra Bascuñán, $S$.

indirect object in Spanish 38 187ff

Bech, Annelise

knowledge representation in MT

10118

Bech, Svend

translating for EU 34 293-297

Becker, A.

silences in communication $\mathbf{2 8} 81$

Beier, Heidrun

gender problems in translation $\mathbf{1 2}$

$59 \mathrm{f}$

Béjoint, Henri

definitions

terminological vs terminogra-

phical 1815

term measurement $37151 \mathrm{ff}$ 
Bell, A.

miscommunication 1582

Bell, Roger T.

translation 12 10, 16202

Beltran-Vidal, D.

LSP 37158

Ben-Amos, D. genre 2394

benchmarking

English 32150

Beneke, Jürgen

technical language $\mathbf{3} 209$

Benmaman, Virginia

legal interpreting 20 216, 226

Benoit, William L.

theory of crisis communication 38 88f

Bereiter, Carl

models of text composition 35190

Berenstein, I.

communication $15106 \mathrm{f}$

Berge, Kjell Lars

urban sociolinguistics 9 211-216

Bergenholtz, Henning

contrastive dictionary grammars 38130

a dictionary for gene technology

10121

lexicography vs terminology $\mathbf{1 8}$

13f, 16, 28

Berke, C.

advertising 23100

Berkenkotter, Carol

genre 16 49f, 54, 23 94, 3750 (def)

definition of 2396

expectations 3752

and text analysis $\mathbf{1 6} 42$

Berrendonner, Alain

connectors 593

Berteloot, Pascale

legal French in France and in the

European communities 31221

Bessé, Bruno de

definitions encyclopedical vs terminological

1826

terminological vs terminogra-

phical 1815

Best Western Denmark 2762

Bever, T.G.

acceptability and grammaticality

814

TRICS 646

Beveridge, Barbara J.

legal English 31 220f

Bhatia, Vijay K.

analysing arbitration laws 329

genre 10 11, 16 47f, 54, 56f, 19

207-238, 23 16, 25 77, 28 118,

3132 (def), 68

communicative purpose 2578

company brochures $\mathbf{2 3} 19$

the legal discourse 22 113f, 3278

letters of application 31 107f

qualifications 3257

textual analysis 32171

Bialystock, Ellen

bilingualism 15149

translation $3152 \mathrm{f}$

bias 12 146f

Bible

The Bishops' 35287

The Church 35287

The Geneva 35287

King James 35 287-290

Bible translation 12 11, 35 287-290

bigraphs 13 187ff

Bilingual Access Line $\rightarrow$ BAL

Bilingual Skills Certificate 12134

bilingualism 15144 (def)

control and competence 15149

research 15 143-152

cognitive 15 145-148

bilinguals

early $15144 \mathrm{f}$

late 15144

Binding and Control, Theory of $\mathbf{1 6}$

196 
binomials 9 54, 58, 3259 (def), 80

(def)

Binon, Jean

lexicography 37156

biomonitoring 3610

Biotec-Lexinome 18 112-119

Biotech.ES 871

Birmingham Corpus 7 71-74, 13 19f

Bitzer, L.

genre 2394

recurrent rhetorical situations $\mathbf{1 6} 41$

black box 3713

Black, Max

the interaction theory 20167

metaphors in dictionaries 20169

Blair, J. Anthony

the relevance problem 5 57-59

Blair, Tony

New Labour 29315

Blakemore, Diana

discourse particles $578 \mathrm{f}$

Blampain, Daniel

terminology 1828

blending $\rightarrow$ conceptual blending;

visual blends

Bloch, J.

Chinese academic writing $2524 \mathrm{f}$

plagiarism 2814

Blum-Kulka, S.

negotiation 1544

speech acts 1534

blurred edges 21228

BNC $\rightarrow$ British National Corpus

Boas, Franz

modern conception of culture in anthropology 38107

Bohr, Niels

description of nature 16178

Boiarsky, C.

cross-cultural miscommunication

1566

Boisson, Claude

translation 37158

Boje, Frede

typologizing phrases $\mathbf{1 0} 120$
Bokmål 1054

Bolter, Jay D.

pictorialization of writing 24 95-98

Bon, F.M.

communicative grammar of

Spanish 12 189-192

Bond, M.H.

cultural impact on international

business success 1554

book information 31 66f

Boolean categories 9 45, 62

Borden, S.L.

apologies 38 91ff

Bordwell, David

dimensions of film editing 34207

bottom-up processing 1225

Bourdieu, Pierre

critique against structuralist linguistics $\mathbf{3 8} 109$

Bowen, David

community vs court interpreting

20215

Bowen, Margareta

community vs court interpreting

20215

Bowers, Frederick

field of statute $\mathbf{3 2} 100$

Bowers, J.W.

lexical diversity $\mathbf{1 1} 16$

Boye, Kasper

evidentiality in Danish 31 236-239

Boysen, G.

syntactic information in dictio-

naries 8103

Braasch, Anna

text corpora and MT 10119

Bradac, J.J.

lexical diversity $\mathbf{1 1} 16$

Braine, G.

plagiarism 2815

brands $2735 \mathrm{ff}$

bank 27 40ff

euphoria 27 39f

information 2738

mission 27 38, 41 
project 2738

prototypes 27 37-40

Braun, Peter

international proverbial sayings

3548

Bravo, D.

confianza 31 126f

Spanish face in business interac-

tions 31126

Brazil's Arbitration Law

vs UNML 32 45-72

Brekke, Magnar genre knowledge and LSP comprehension 23 9f

LSP research 19 251-261

Bresnan, Joan

lexicalism 16192

Brisk, Maria Estela

communication expectations 3762

British $\rightarrow$ English

British National Corpus 1355

British Standard Organization 621

Brockmeier, J.

greenspeak 2758 (def)

Bros-Brann, E.

interpretation

response to H.C. Barik 1275

Brown Corpus

the verbs begin and start 13

105-120

Brown, Penelope

dialogues $\mathbf{3 8} 45$

face want 2776

face-threatening acts 2776

facework vs politeness strategies

2776

in-group jargon 2878

politeness $2775 \mathrm{f}$

Brown, S.C.

environmental discourse 2758

Brown, Stephen

the paradoxes of green marketing 2761
Brown, V.J.

scientific and technical discourse 2372

Bruce, Gösta

prosody 3323

Bruckman, Amy

MediaMOO 2486

Brunet, Étienne

loan words in French LGP dictionaries 37155

Brunner, $\mathrm{H}$.

blurred genres 2395

BSC 12134

Budin, Gerhard

ontology-driven translation ma-

nagement $38225 \mathrm{f}$

Bühler, Karl

language functions 1783

metaphors 2471

Organon Model 17 83f

Bülow-Møller, A.M.

negotiations cross-cultural 1515

simulated 11 9f

Bullock, Carolyn

interpreting $20223 \mathrm{f}$

Burke, Kenneth

entitlement 37 17-19

terministic screens 37 17-20

Bush, Vannevar

hypertextuality 2489

business $\rightarrow$ negotiation

business communication 31 9-12

cross-atlantic 15 53-76

concepts of politeness and time

15 53-76

Anglo-Germanic and Latin

(=Hispanic/French etc.) 15

53-76

cultural impact on 15 53-76

foreign language needs

in companies 31 17-25

Denmark 31 13-15

German language needs 31

13-28 
research on 31 15-17

international business

and language use 38 105-114

language tests $\mathbf{3 1} 16$

business documents

reading and writing 1566

business interaction

handling of complaints in cross-

cultural 21 67-91

business letters 15 67f, 3757

American-Mexican 37 53f

Chinese 3758

cultural expectations of 37 53-55

Japanese 37 53, 57f, 60f

Mexican 37 53f, 60f

business manager

language/culture knowledge 15

71-73

personality $1569 \mathrm{f}$

business rhetorics 27 ff, 55-81

business environmental discourse 2759

Bußmann, Hadumod

LSP dictionaries 3 131ff

Butler, C.S.

indirect speech acts 28114

butterfly 12 144-147, 152

butterfly catastrophe 12145 (fig),

146 (fig)

C

Cabré, M. Teresa

lexicology

vs lexicography 37152

vs terminology 1822

terms $37151 \mathrm{f}$

CALD $\rightarrow$ Cambridge Advanced

Learner's Dictionary Online

Calvet, L.-J.

terminology work in French-

speaking countries 1824

Cambridge Advanced Learner's

Dictionary Online 3415
Campbell, Charles P.

business letters

Chinese and Japanese $3758 \mathrm{f}$

cultural expectations of $\mathbf{3 7} 53$

near-copying 2840

plagiarism $2814 \mathrm{f}$

Campbell, Stuart J.

translation 2617

Camus, Albert

junction 26 71-77

Candlin, Christopher N.

analysing arbitration laws 329

Carlson, Rolf

prosody 3324

Caro, Martinez

listing 25103

strict opposition and parallelism

25101

Carr, Silvana E.

interpreting in legal, health and social service settings 20 211-231 three-tiered health care interpreter system 20229

Carras, Catherine

French as a foreign LSP 37158

Carrol, John S.

negotiation 159 (def)

Carson, J.

plagiarism 2814

case $\mathbf{1 7} 96$

case descriptions 32 80f (def)

case filter 16197

case theory $\mathbf{1 6} 196$

casuistry $\mathbf{3 8} 92$

catastrophe theory $12142 \mathrm{ff}$

categories 1858

Boolean 9 45, 62

cognitive 1857

vs concepts 1858

ontological 1857

categorisation 1855

vs classification 1855

the problem of categorising

metaphors $\mathbf{2 4} 68 \mathrm{f}$ 
Catford, J.C.

concept of equivalence $\mathbf{1 2} 11$

translation 1212 (def), 68

Caucasian languages 35 60f

CCI 12134

CCSARP-method

vs natural data method $1537 \mathrm{f}$, $43 \mathrm{f}$

CCSARP-project 15 34f, 43

criticism against $\mathbf{1 5} 37$

Celtic languages 3556

Cenkova, J.

interpretation research policy 22

$162 f$

Centre de terminologie et de néolo-

gie 8 109, 1815

CERA 12 65-67, 69f, 82

Chaney, L.

intercultural communication 15 74f

Channell, J.

vague language $\mathbf{2 8} 62$

Charbonnel, Nanine

metaphors in dictionaries 20169

Charrow, R.P.

legal language 911

Charrow, W.R.

legal English 911

chat programs 24 93-95

Chatterjee, $\mathrm{P}$.

passive advertising 23101

Chen, T.G.

Chinese academic writing $2524 \mathrm{f}$

plagiarism 2814

Chenoweth, N.A.

corrective feedback 1593

Chesher, Terry

community interpreting 20214

in Australia 20224

Chesterman, Andrew

knowledge 38233

memetics of knowledge $38222 \mathrm{f}$

translation $\mathbf{3 1} 203$

studies

definitions 31 198f

theory
history of $\mathbf{1 2} 69$

Chi, L.

plagiarism 2814

Chinese

academic writing $2525 \mathrm{f}$

business letters 3758

vs French at the utterance level

13 351-353

intermediate learners of German unsatisfactory dictionary situation $30216 \mathrm{ff}$

Chomsky, Noam

core language 38190

descriptive adequacy 16178

Extended Standard Theory 16195

functionalism 16 179f

grammatical filter 813

grammaticality and acceptability

8 9f

lexicalism 16191

Minimalist Programme 16199

nominalization 10 12-15

purpose of language 16179

purpose of linguistic description

16200

Trace Theory 16 195f

Christensen, Annie

instruction manuals 10 117f

Christie, F.

genre 16 44f, $53 \mathrm{f}$

Christmann, Ursula

comprehensibility of texts $\mathbf{3 6} 25$

Chubin, D.E.

English academic writing 2524

Chun, A.E.

corrective feedback 1593

chunk 14134 (def)

claims 15 118-120, $128 \mathrm{f}$

peripheral 15 120, 124

Clas, André

value of linguistic signs 37152

classification 1855

vs categorisation 1855

clauses 17 29f, 33 
discontinuous 17 40-49

see also syntax

climbing 19 104, 108f

clitic doubling 19100

clitic-doubled constructions 38189

(def)

in Spanish 38 187-202

cliticization

grammaticalization of 20 125-128

in Romance languages 20 119-125

clitics 20117 (def)

anteposition and multiple depen-

dancy relationships $\mathbf{2 0} 128$

position of

in Romance languages 19 93-111,

20 115-137

simple 20117

special 20117

subclasses of $\mathbf{2 0} 118 \mathrm{f}$

see also emphiclisis; enclisis;

proclisis

Clyne, Michael

translation 32170

co-operative principle 695

Cobuild 7 71f, 30 223f

Cocchini, $\mathrm{M}$.

community interpreting 20228

Cockney 17 226-228, 38 175ff

boundaries between Cockney and RP 38181

code 536 (def)

-switching 15 45f, 17 218-220

lexis 28 59-79

in-group 28 59-79

code of conduct 12 173f

Codina, Marta X.

promoting legal Catalan 31222

Coggle, Paul

Estuary English 38 181f, 184

cognition (human) 6 32-34

cognitive categories 1857

cognitive pragmatics 38232

coherence

intratextual $1438 \mathrm{f}$

Kohärenzregel 16 206, 214
Collins COBUILD English Language Dictionary 771

biotechnological terms in monolingual dictionaries 18 103-107

Collins, H.M.

knowledge 25117

collocability 13 22f, 28f, 29 (fig)

collocations 7 130-137, 34 71-76

grammatical 7132 (def), 132f

lexical 7 133-136, 133 (def)

open 7133,136

COLT 13 55-69

application of TEI mark-up 13

61-64

laughter (transcription of) $1360 \mathrm{f}$

pauses (transcription of) 1360

taboo words 13 67f

tonic marks (transcription of) $\mathbf{1 3} 59 \mathrm{f}$

combining forms 2516 (def)

vs affixes 25 17-19

commencement 3287 (def)

in the Malaysian Arbitration Act

3287

commissives 9 84-92, 105

communication $\mathbf{6} 95,15$ 106ff, 17

97-101, 38232

activities 3813

analysis 27 17-23

back-channeling 3 258-260

behaviour

Spanish and Scandinavian 3

247-264

bilingual

with an intermediate translator /

interpreter 1237 (fig)

business 31 9-12

cross-atlantic 15 53-76

concepts of politeness and

time

Anglo-Germanic and Latin

(=Hispanic/French etc.) 15

53-76

cultural impact on 15 53-76

foreign language needs

in companies 31 17-25 


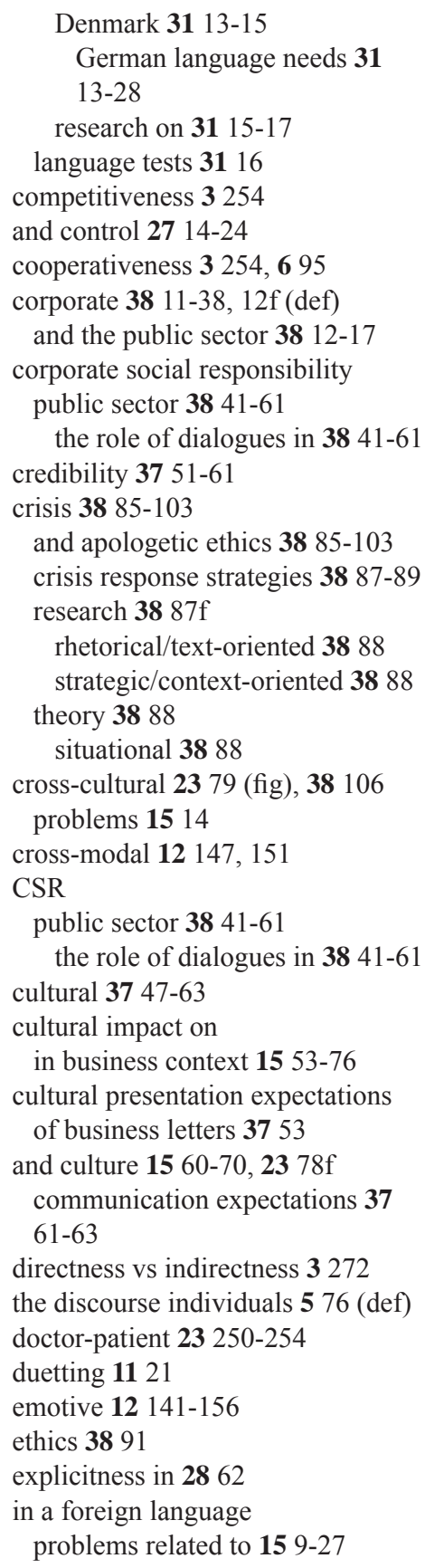

forums 37 50-52

German 3457

green

Danish hotels 2763 (fig)

group membership and selfassertiveness $3252 \mathrm{f}$

the inferential account of 5 39-41

integrativeness 3254

and interaction 17 67f

intercultural 11 7-10, 15 74f, 31

119-133, 37 52f

interaction 3 247-264

taxonomy of $\mathbf{1 1} 8$

theoretical approaches 31 122-127

intercultural layer 11 19f

intergroup layer 11 16-18

interlanguage layer 11 20-22

interlingual 846

interpersonal 3 298-301

interpersonal layer $\mathbf{1 1} 15 \mathrm{f}$

and interpretation 12 36-41

Japan 14140

legal

between experts and non-

experts 36 9-12

linguistic monitoring of 36 9-12

LSP 25 66-68, 28 7, 37 9-20

management control 27 9-28

in marketing $2380 \mathrm{ff}$

marketing 27 31-52

maxims 3769 (def)

mis- 12 176f, 15 66, 82 (def), 3759

affective $\mathbf{1 5} 82$

incipient 15 81-108

referential 1582

relational 1582

sociocultural 15 97-106

types of $\mathbf{1 5} 86-92$

discourse structural 15 88-97, 106

lexical 15 86-88, 92-97, 106

models of

code model 5 36-39

interactionistic model 14114

language game model 3271 
model of successful communication 1231 (fig)

transfer model $14113 \mathrm{f}$

monolingual 1237 (fig)

in multilingual firms 38 109-114

in negotiations 3 209-215

in an open social systems appro-

ach 3814

oral 31 47-64

competence areas 3150

discourse and textual compe-

tence $\mathbf{3 1} 50$

linguistic competence 31 50, 55

pragmatic competence 3150

socio-cultural competence $\mathbf{3 1}$

50,55

strategic competence 3150

modal expressions $3156 \mathrm{f}$

policy 37 95-113, 38 15f

of Danish public institutions 38

$18 \mathrm{ff}$

essential elements 3822 (fig)

content elements 3822 (fig),

28-32

elements of textualization $\mathbf{3 8}$

22 (fig), 24-28

general 37105 (def)

interlingual 37106 (def)

intralingual 37106 (def)

vs language policy $37111 \mathrm{f}$

mediational properties 38 14-17,

32-37

as a process $3820 \mathrm{f}$

as a product $3821-24$

analysis 38 24-32

in public communication 38 11-38

specific 37106 (def)

the term $3795 \mathrm{ff}$

presuppositions of $\mathbf{1 2} 32$

principles of $\mathbf{3} 251 \mathrm{f}, \mathbf{5} 66 \mathrm{f}$

problems 15 82ff

and product names $2389 \mathrm{f}$

in public 38 7-9

public 38 7-9, 66-69

legitimacy $3813 \mathrm{f}$ purpose 19 214-219, 232-234, 28

121, $3138 \mathrm{f}$

the recipient 576 (def)

references in 12 29-36

requestive message production

in the British Political Consul-

tancy Industry 29 313-324

semi- 12217

Scandinavian 12216

settings 12 122-124

silences 28 81-104

conventional 2883

discreet $\mathbf{2 8} 83$

incidental 2883

innovative 28 87-89

institutional $2886 \mathrm{f}$

manipulative 2883

meaningful 28 87-89

presuppositional 2883

speech act $\mathbf{2 8} 83$

textual 2883 (def), $90 \mathrm{f}$

and the conference abstract $\mathbf{2 8}$

95-99

in scientific research articles

28 81-104

typology of $2882 \mathrm{f}$

situation 32174

the speaker 576 (def)

and standardization $2379 f$

strategies 15 113, 3769 (def)

achievement 1592

cooperative 1592

non-cooperative 1592

in the financial sector 27 31-52

reduction 1592

cooperative 1592

non-cooperative 1592

successful

idealised model of 1231 (fig)

tactics

distributive 1512 (def)

integrative 1512 (def)

technical 37 9-20, 37-41, 38226

and translation 12 36-41, 23 77-91

verbal and non-verbal 37111 
see also conversation; conversational organization; cooperativeness; dialogues; distributiveness; duetting; face; footings; integrativeness; marketing; meaning; moves; negotiations; style; turntaking

communication research

American vs European 1511

on bargaining 1513

on cross-cultural negotiation $\mathbf{1 5}$

11-13

communities of practise 37 14-17, 20

competence

linguistic 14 57f, 60f

competitiveness 3254

complaints 21 72-74

customer

responding to 21 77-84, 84 (fig)

evasive strategies 2177

everyday

responding to 21 72-77, 77 (fig)

rejections $2182-84$

remedial acts 21 75f, 81

thanking for complaints $2179 f$

complements $\mathbf{1 0} 12 \mathrm{f}$

the complement rule 1016

complementizer deletion $\mathbf{1 6} 188$

(fig)

complementizer placement $\mathbf{1 6}$

186 (fig)

Complex-NP-Constraint 817

compliance 29 313-324

componential analysis 1217

compositionality $\mathbf{1 3} 21 \mathrm{f}$

types of 13 24-26

compounds 2515

neo-classical compunds 25 15-17

comprehensibility research $3767 \mathrm{ff}$

$\log$ files of reverbalization pro-

cesses 37 67-89

methods in $\mathbf{3 7} 68$

thinking-aloud protocols in 37

67-89

compromisers 13 157-166, 159 (def) the compromiser paradigm 13

159-165

compromising 12147

Compuflex localisation 36203

computational architecture 6 31-33

computer

advertisements 24 31-55

commercializing the desktop 24

144-151

desktop metaphor

conceptual mapping of 24145

interface as 24145

metaphors 24146

as prosthesis 24 15-29

see also IT

Comrie, B.

Accessibility Hierarchy 821

Comtec Translations 16 210-213, 219

Conceição, Manuel C.

reformulation phenomena in LSP

texts 37154

concepts 1858,88

vs categories $1858 \mathrm{ff}$

defining 8130

conceptual blending 24 138-144, 157

schema 24140 (fig)

theory $24138 f$

see also visual blends

conceptual integration 24 139f, 156f

theory 24140

confianza 31 126-130, 132

configurationality parameter $\mathbf{1 0} 24 \mathrm{f}$

conflict management

in price negotiations 15 111-139

Confucian dynamism 15 55f

connectionism $25120 \mathrm{f}$

connectors

formality of 5 89-91

French

tandis que 25 93-109

functions of 5 79-84

iconic functions 5 83f, 88

pointing functions 5 81-83, 88f

the logic and pragmatics of 5 69-93

meaning of $569 \mathrm{ff}$ 
Connor, U.M.

plagiarism 2815

"consensus first" principle 15 115f,

124,126

constituents

structure 10 9-11

constitutive rules 32106

construction kit 1768 (def)

content structure 2579 (def), 85-90

Contente, Madalena

a multilingual medical dictionary

$37157 f$

contexts

in bilingual dictionaries 28 202-207

contextual effects 5 43-47

contextualisation cues $\mathbf{2 8} 60$ (def)

continuity principle 34204

contracts 985 (def)

oral vs written 1566

control 1769 (def)

convergent subjects 19253 (def)

conversation

cultural mismatch in 3 247-264

maxims of 5 40f, 47, 64, 78, 6 95,

31199

conversational organization 3 258-262

Cook, Guy

translation and language teaching

30 199f

Coombs, W. Timothy

crisis communication research $\mathbf{3 8}$

$87 \mathrm{f}$

crisis response strategies $\mathbf{3 8} 88$

SCCT 3888

cooperativeness $\mathbf{3}$ 254, 695

Copeland, Jack

human and machine memory 25120

copulatives

dynamic $3428 \mathrm{f}$

Corbin, Danielle

courses in lexicography $\mathbf{1 8} 19$

Corbin, $\mathrm{P}$.

derivations in dictionaries 8105

Cornelissen, Joep

communication activities $\mathbf{3 8} 13$ corporate communication 38 11-38,

$12 \mathrm{f}$ (def)

and the public sector 38 12-17

corporate social responsibility $\mathbf{3 8} \mathbf{4 1 \mathrm { f }}$

developing and implementing

in a dialogical process 38 53-58

corpus linguistics 139

concepts 29287

data 29287

methods 29287

Corpus-Bench 13 14-17

corrective feedback 1593

off-record 1593

on-record 1593

Corsellis, Ann

community interpreting 20221

Coseriu, Eugenio

tetratomy of language $\mathbf{1 7} 85 \mathrm{f}$

COSMAS 3459

Costa, Rute

specialized text corpora 37154

cotexts

in bilingual dictionaries 28 202-207

court 3286 (def)

decisions 36 17f

German 36 15-39

proceedings 36132

Courtright, J.A.

lexical diversity $\mathbf{1 1} 16$

courtroom discourse 36 129-154

amount of speech $36148 \mathrm{f}$

hedges $\mathbf{3 6} 150$

interruptions 36149

pauses and pause fillers 36150

questions 36150

simultaneous speech 36 149f

supporting utterances $\mathbf{3 6} 150$

see also legal discourse

Crandall, J.A.

legal language 911

credibility precondition $\mathbf{1 5} 115$

Cressy, David

literacy of English women 13141

criminal trials

Swedish 
male and female witnesses' speech in 36 129-154

crisis communication 38 85-103 and apologetic ethics 38 85-103 crisis response strategies $\mathbf{3 8} 87-89$ research 38 87f

rhetorical/text-oriented $\mathbf{3 8} 88$ strategic/context-oriented $\mathbf{3 8} 88$ theory $\mathbf{3 8} 88$ situational $\mathbf{3 8} 88$

crisis management 38 89-97

criticism

in academic writing 25 23-44

Chinese 25 24f

Czech 2525

English 2524

German 2525

Malaysian 2525

Polish 2525

Crocker, Jean

academic writing 288

Cronin, Michael

localisation 36203

Cross, Patricia K.

teaching grammar $\mathbf{3 1} 98$

cross-modality

interactional 12147

cross-reference (Dik's terminology)

$38192 f$

Cruttenden, A.

definition of nucleus 13236

Crystal, David

Estuary English 38 182, 184

LSP dictionaries 3130

CSR communication 38 41-61

$\mathrm{CTN} \rightarrow$ Centre de terminologie et de néologie

cues

emotive 12 149, 150 (fig)

Culpeper, J.

language interaction 36153

men's and women's language 36 147

cultural filters in cross-cultural communication

2379 (fig)

cultural misunderstanding 15 53-76

culture 1554 (def), 38 222, 231 (def)

arguments

across national/cultural groups

15125 (fig)

and communication 15 60-70, 23

78f, 37 47-63

and communication expectations

37 61-63

cross-cultural communication 23

79 (fig)

cultural constellations in text and

translation 38 230f

cultural expectations

of business letters 37 53-55

related to genres 37 47-63

cultural systems 38 107f, 231

cultural values $15114 \mathrm{f}$

dia- 1440

international 1449

differences between Spaniards

and Scandinavians 15 115-117

digressive 1557

directive 1557

floor-giver 3258

floor-taker 3258

idio- 1440

impact on communication in

business context 15 53-76

importance of

in the EU and NAFTA areas 15

55-60

influence of

in Danish and British sales brochures 23 77-91

in Danish and French company

brochures 25 217-226

institutional 32174

interaction psychology $3269 f$

and interpreting/translation $\mathbf{1 4}$

$39-41,139 f$

and language 1219

and style 23 86-89 
linear 1557

and management 15 55-60, 71

models

systemic 37 12-15, 12 fig, 13

fig

modern conception af $\mathbf{3 8} 107$

monochronic/polychronic diffe-

rences 15 57f, 72

national society 1440

and negotiations 3 198-202

para- 1440

product settings $2382-85$

research

cross-cultural 118

intercultural 118

as a system 37 10-14, 11fig,

12fig, 13fig

through text and image 25 217-226

see also communication; ne-

gotiations

Curl, Tracy

requests $3870 \mathrm{f}$

Cusin-Berche, F.

specialized dictionaries $\mathbf{1 8} 21 \mathrm{f}$

Cutting, Joan

academic writing 288

implicit use of language 2862

cybermarketplace 24 144, $156 f$

CYC 25122

Czech

Czech academic writing 2525

D

Dagut, M.

lexical gaps 28199

Dahl, Trine

LSP research 19 251-261

Dam, Helle V.

intercultural communication, negotiation and interpreting 22165 knowledge systems and translation $38221 \mathrm{ff}, 233$

semantic networks $38229 f$ translation studies

definitions and names 31 202-204

Danaher, Paul

translation theory is useless $\mathbf{2 6} 99$

Danet, Brenda

legal language 6 66ff

English 911

Danish

articles about business cycles

metaphors in 24 57-79

aspect

vs English 10 46f

auxiliaries

vs English 7 164f

brewery brochures 27 215-227

communicative behaviour

vs Spanish 3 247-264, 267-278

company brochures

and French 25 217-226

differences between Spaniards

and Scandinavians 15 115-117

distributive behaviour 3 273-275

English grammars for Danes 17

113-120

evidentiality 31 236-239

German language needs in

Danish companies 31 13-28

medical hybrids 25 19f

mood

vs English 7 163-174

negotiation interaction

vs Spanish 3 267-278

negotiation language

vs English 11 11-23

negotiations

vs Spanish

conflicts and consensus 15

111-139

strategic preferences negotia-

ting price 15 130-134

PPIs 25 69f

sales brochures

vs British 23 77-91

tense

vs English 7 163-174 
translation

Danish-English 8 85-91

Danish Dictionary, The

$\rightarrow$ Den Danske Ordbog

Danish Internet Dictionary, The

$\rightarrow$ Den Danske Netordbog

Danish Public Employment Service

38 68f

guidance interviews 38 70-73

requests 38 70-73

Dansk-engelsk ordbog (V/B) 12213

Dansk-engelsk Ordbog. Undervisning review 7 117-121

Dardel, R. de

prosodic laws 20126

Darò, V.

simultaneous interpreting skill components in 22164

Dascal, M.

communication $15106 f$

data 2 179-185

deceptive data of language interpretation 3 287-308

Davenport, Mike

English sentence analysis 12186 189, 1711

Davidsen-Nielsen, Niels

English

aspect 1476

pronunciation 10 125-127

sentence analysis 1711

tense and mood 7 163-174

Dawrant, Andrew

Effort Models 23158

Day, R.R.

corrective feedback 1593

declarations 6 72-74, 73 (def), 88, 9

14

decoding 5 36-39

deep structure 16186 (fig)

definition rules (in legislation) 32101

definitions

encyclopedical vs terminological

1826

functional $37156 \mathrm{f}$ terminological 37156

vs terminographical 1815

and translation studies 31 197-207

degree modifiers 13157 (def)

Déjean Le Féal, K.

simultaneous interpreting 1280

Delabastita, Dirk

translational norms 1481

deletion 17 277-280

semantic-pragmatic analysis of

17278

syntactic analysis of $\mathbf{1 7} 278$

Deleuze, Gilles

transversality 24100

DeLillo, Don

Underworld 34 199ff

Delphi-method 3117

Deming, W.E.

quality management 16208

Den Dansk-Engelske Regnskabs-

ordbog 34 103-112

design 3442

Den Danske Idiomordbog

design 3442

Den Danske Netordbog

analysis of log files 34 117-140

computer-related terms $34137 f$

design 34 41-47

dyslexia 34130

lemma lacuna $34136 f$

lemmas not searched for $34139 f$

non-existing words 34 133-135

spelling mistakes affected by pronunciation 34 130-132

verbs

imperative 34 128-130

passive $34127 \mathrm{f}$

Den Danske Ordbog

text corpus and corpus retrieval

system of 13 11-17

Den Danske Regnskabsordbog design 3442

Dendien, J.

computerization of dictionaries 8 108 
Depecker, Loïc

terminological definitions 37156

Depierre, Amélie

level of specialization of LSP

texts 37154

description 16 178-180, 31 65-79

descriptive adequacy 16 178ff

place 3167

descriptivism 17 113-120

descriptive pragmatics $2171 \mathrm{f}$

desktop publishing 36213

diachrony

micro- 3465

diaculture 1440

international 1449

dialects 38 173-185

Cockney 17 226-228, 38 175ff

boundaries between Cockney

and RP 38181

and language 36200

London-English 38 175f

metropolitan sub-variety $\mathbf{1 7} 227 \mathrm{f}$

modern dialects $\mathbf{1 7} 215$

modern nonstandard dialects $\mathbf{1 7} 215$

Oxford accent 17 213ff

vs socio-regional speech 17

213-234

regional prestige 17 224-226

traditional dialects $\mathbf{1 7} 215$

Upper-crust 17 226-228

dialogism $14113 \mathrm{f}$

dialogues 3 270f, 12 36, 3843

(def), 44-48, 44 (def)

-change model 3855 (fig)

asymmetrical $\mathbf{3 8} 45$

between the Danish State and

Danish employers 3850

CSR 38 48-51

initiatives and responses in 3260 -

262

kinds of $\mathbf{3 8} 45$

in public sector CSR communication 38 41-61

structure and function 38 46-48
Diamantis, D.

the commercial green discourse 2762

Dichy, Joseph

S-specifiers 37156

dictionaries 449 (def), 38 119-122

accounting

internet 34 81-115

comparing $34109 f$

general characteristics 3489 -

102

data fields 34 95-102

antonyms 34101

collocations and phrases

34102

definitions 34 95-100

grammar and spelling 34

$101 \mathrm{f}$

selection of lemmata $\mathbf{3 4} 95$

synonyms in English vari-

eties 34 100f

nature of 34 84-88

articles

single 28207 (def), 207f

synopsis 28207 (def), 207f

bilingual

context and cotext in 28 195-208

equivalence 28 195-208

adressing 28 203-206

over 28204

under 28203

zero $28203 \mathrm{f}$

congruence $28196 f$

divergence 28 197-199

lexical 28197

semantic 28198

full $28196 f$

lexical gaps 28 199-202

linguistic gaps $\mathbf{2 8} 200$

referential gaps $\mathbf{2 8} 200$

partial 28 197-199

surrogate 28 199-202

zero 28 199-202

information retrieval in $\mathbf{2 8}$ 202208 


\begin{tabular}{|c|c|}
\hline classification 18 96-98 & internet 3485 \\
\hline collocational 693 & designing 34 37-54 \\
\hline criticism $695 f$ & $\log$ files 34 117-140 \\
\hline descriptive adequacy $1058-62$ & language 1896 (def), 31 177-185 \\
\hline descriptive aspects $1053-64$ & genuine purpose 31 177-185 \\
\hline a dictionary for gene technology & learner's 6 93, 30 215-233 \\
\hline 10121 & amount of lemmata in $\mathbf{3 8} 219$ \\
\hline dictionary grammars 38 119-134, & bilingualised 35 281-286 \\
\hline $120(\mathrm{def})$ & Chinese intermediate learners \\
\hline contents of $\mathbf{3 8} 122 \mathrm{f}$ & of German \\
\hline contrastive 38 130-133 & unsatisfactory dictionary situ- \\
\hline model of description 38127 & ation $30216 \mathrm{ff}$ \\
\hline structure of 38 123-127 & different types of articles 30 \\
\hline alphabetical $38123 \mathrm{f}$ & $215-233$ \\
\hline thematical 38 123-125 & irregular flexion variants in $\mathbf{3 8} 219$ \\
\hline and types of dictionaries $\mathbf{3 8}$ & LGP \\
\hline $127-130$ & French \\
\hline bilingual 38 128-130 & loan words in 37155 \\
\hline general monolingual & specialized entries in 37157 \\
\hline for learners 38128 & linguistic vs encyclopedic know- \\
\hline for native speakers 38 127f & ledge in $698 \mathrm{f}$ \\
\hline electronic 34 7f & look-up strategies 35 281-286 \\
\hline Bantu languages 34 20-32 & LSP \\
\hline vs paper $347 \mathrm{f}, 16-19,117 \mathrm{f}$ & of biotechnological terms $\mathbf{1 8}$ 108ff \\
\hline South Africa 34 11-33 & grammatical information in $\mathbf{3}$ \\
\hline encyclopedias 1896 (def) & $125-140,10122$ \\
\hline encyclopedic 1896 & the ideal LSP dictionary 6 91-107 \\
\hline field labels 693 & lexical information in $\mathbf{1 0} 122$ \\
\hline functions $\mathbf{6} 96, \mathbf{1 8}$ 98-101, 31 & for translation 6 104-106 \\
\hline $171-194,176(\mathrm{def}), 3485$ & use of $\mathbf{1 0} 122$ \\
\hline communication-orientated 31 & vs web resources 37153 \\
\hline 176,3485 & metaphors in 20 169f \\
\hline vs functionalities $3438 \mathrm{ff}$ & monolingual \\
\hline knowledge-orientated 31 176f, & vs bilingual $692 \mathrm{f}$ \\
\hline 3485 & biotechnological terms in $\mathbf{1 8}$ \\
\hline modern theory 31 172-177 & $103-107$ \\
\hline text dependent 3485 & non-monolingual $692 \mathrm{f}$ \\
\hline text independent 3485 & normative aspects $\mathbf{1 0} 53-64$ \\
\hline text production and translation & orthoepic/orthographic 693 \\
\hline $18107-112$ & pragmatic items 693 \\
\hline general vs special 693 & root 693 \\
\hline genuine purpose 31176 (def) & semiotax $28206 f$ \\
\hline vs grammars 38 119f & single-field 3484 (def) \\
\hline grammatical information in $\mathbf{3}$ & synonym 693 \\
\hline $125-129,34$ 76-78, 38 119-134 & terminological 6 96-102 \\
\hline
\end{tabular}


as texts 695

vs thesauri 20 169-178

thesis about four dictionaries per

language pair 6102,8104

for translation 6 96-98, 102-106

typologization 6 92-94, 106

functional 6 92-94, 106, 31 182ff

phenomenological 6 92-94,

106, 31 178-185

Wiegand 31 178-185

user situations

communication-orientated $31174 f$

knowledge-orientated $31173 \mathrm{f}$

users 6 96, 18 98-101

experts 18102 (def)

laypeople 18101 (def)

research on $34119 f$

dictionary protocols $\mathbf{3 4} 120$

questionnaire surveys $34119 \mathrm{f}$

semi-experts 18101 (def)

user attitude $22102 \mathrm{f}$

user levels 6100

see also lexicography; LSP lexi-

cography; spelling checkers

Dictionary of English Style $7129 \mathrm{f}$

Diderichsen, Paul

topological tradition 12199

Dienhart, John

English sentence analysis 12 186189, 1711

Diggelen, A. van

business letters 1567

digital entanglements 24 93-95

Dijk, T.A. van

superstructures 621

Dik, Simon C.

connectors 2593

discourse 2595

coherence 2596

focal information 25100 (def)

Functional Grammar 38196

markedness 38190

recursion 2596

subject and object alternation $\mathbf{3 8}$ $199 f$
Dillinger, M.L.

interpreters/non-interpreters 1281

direction of fit $\mathbf{6} 72 \mathrm{f}$

directives 6 72, 74-88, 9 84-92, 85

(def), 102, 17 90f, 91 (fig)

directness level of $986 \mathrm{f}$

in English contract law 6 77-83

in everyday conversations 6 83-87

disambiguation 3469

discontinuity

in English 17 37-62

discourse

coherence 25 93-109

global 2596

local 2596

community 2861 (def)

courtroom 36 129-154

amount of speech $36148 f$

hedges 36150

interruptions 36149

pauses and pause fillers 36150

questions 36150

simultaneous speech $36149 f$

supporting utterances 36150

events 2594 (def)

global

decisions $2594 \mathrm{f}$

structures in $2595 \mathrm{f}$

green 27 57-59

commercial 2759

in western societies 2758 (fig)

institutional 36131

gender differences 36 129-154

legal 9 9-17, 22 113-133

across cultures 32 13-33

across languages 32 13-33

multicultural and multilingual

contexts

generic integrity 32 7-11, 13-33

written $9116 \mathrm{f}$

the audience in $9116 \mathrm{f}$

legislative $\rightarrow$ legal

markers of written 17280 (def)

meta-

classification of 31113 (fig) 
particles 5 78f

planned vs unplanned 8 46f

production 25 75-90

scientific

the said and unsaid in $\mathbf{2 8} 81-89$

situational 28 120-125

topical continuity 2596

distribution 36201 (def), 204f

distributiveness 3254

Ditlevsen, M.G.

communication policies $\mathbf{3 8} 8$

divergent subjects 19253 (def)

Dixon, John

gender-based language differen-

ces 36153

Dixon, R.M.W.

syntax and semantics of aspectual verbs 13101

Dobrzynska, Teresa

translation of metaphors 35189

Docherty, G.J.

Estuary English 38175

document management $\mathbf{3 8} 226 \mathrm{f}$

documents

access structure of $\mathbf{1 7} 64$ (def)

virtual 1766 (def)

Dodds, J.M.

interpretation 1278

research and training 22163

Dollerup, Cay

translation

between Danish and English 1477

vs interpretation 1472

Dolmetschwissenschaft 1473

domain-level patterns 30 179-196

dominance theory 36130

Donohue, W.A.

communication research 1512

Dorland's Illustrated Medical Dic-

tionary 3 134ff

Douglas, A.

phases in negotiation 1165

Dow, E.

negotiation strategies 2170
Dowling, G.R

image 2760 (def)

downgraders 1546

Dressen, Dacia F.

academic writing 288

Drew, Paul

requests $3870 \mathrm{f}$

Dreyfus, Hubert

equating humans with machines

25117

drift 38192 (def)

dubbing 17 244-250

Dubslaff, Friedel

interpreting

simultaneous 1412

Ducrot, Oswald

connectors $570 \mathrm{ff}$

silences in communication $\mathbf{2 8} 81,83$

DUDEN: Das große Wörterbuch

der deutschen Sprache

biotechnological terms in mono-

lingual dictionaries 18 103-107

Dürr, François

LSP communication $37158 \mathrm{f}$

duetting 1121

Dufour, Aideen

interpreting in legal, health and

social service settings 20 211-231

Dumitrescu, Domnița

echo questions 13331

Dungworth, D.

special language 2566 (def) vs general language 2566

Dupont, Christophe cross-cultural negotiations 1515

Duszak, A.

German, Polish and Czech academic writing 2525

Dutch

requests

vs French 1562

Duval, A.

bilingual lexicography 8102

Dynamic Model of Simultaneous

Interpreting 14139 
dynamics

trimodal 12145

Dyrberg, Gunhild

legal text corpora 8128

$\mathbf{E}$

E-language 38223

ear-voice span 10 45, 14136

$\mathrm{EC} \rightarrow \mathrm{EU}$

EC market

size of 15 59f

economics texts

English 23 25-40

external variables $2336 \mathrm{f}$

internal variables 23 28-36

focal terminology 23 32-34

lexical aspects 23 29-32

pronouns in 23 34-36

ecospeak 2758 (def)

Edelsky, C.

defining floors 1163

Edmondson, W.

complaints 2172

effort models 12 79f, 23 153-169

competition hypothesis $23156 \mathrm{f}$

tightrope hypothesis 23 159f

efforts 23154

ego-consciousness 3 290-307

Ekedahl, Olof

dBASE program 13126

Elektroniese Wat 34 18f

elementary catastrophes 12144

elexico 34 55-81

-corpus 3457

grammar 34 76-78

information

sense dependent 34 66-78

collocations 34 71-76

criteria for word sense dis-

tinction 34 68-70

meaning and use $3467 \mathrm{f}$

sense independent 34 62-66

conceptual sense relations 3466

diachrony 34 65f regional variety 3466

spelling and spelling variety

3464

word formation and variant

forms 3465

microstructure 34 62-78

pragmatics 3476

principle of modularity 34 60f

Emonds, J.E.

transformations 8 19f

emotional display

divergent preferences for $\mathbf{1 5} 116$

emotional security 12 146f

emphiclisis 20126

enacting formula 6 73f, 88, 914

enclisis 19 95f, 20124

and proclisis

asymetry between $20128 \mathrm{f}$

opposition between 20 124f

Encyclopedia Britannica

medical articles in $\mathbf{1 9} 254$

encyclopedias 1896 (def)

endocentricity constraint $\mathbf{1 0} 14$

Endres-Niggemeyer, Brigitte

abstracting 25127

Engadine

clitics in 19 97-99

engagement 1769 (def)

Engberg, Jan

German and Danish judgments 916

legal communication 3611

semantic networks 38 229f

translation $32170 \mathrm{f}$

and knowledge systems 38

$221 \mathrm{ff}, 233$

studies

definitions and names 31 204-

207

Engelbart, Douglas C.

oN Line System 2489

English

academic writing

criticism in $\mathbf{2 5} 24$

accounting terminology 32 145-164

UK and US 32 149f 


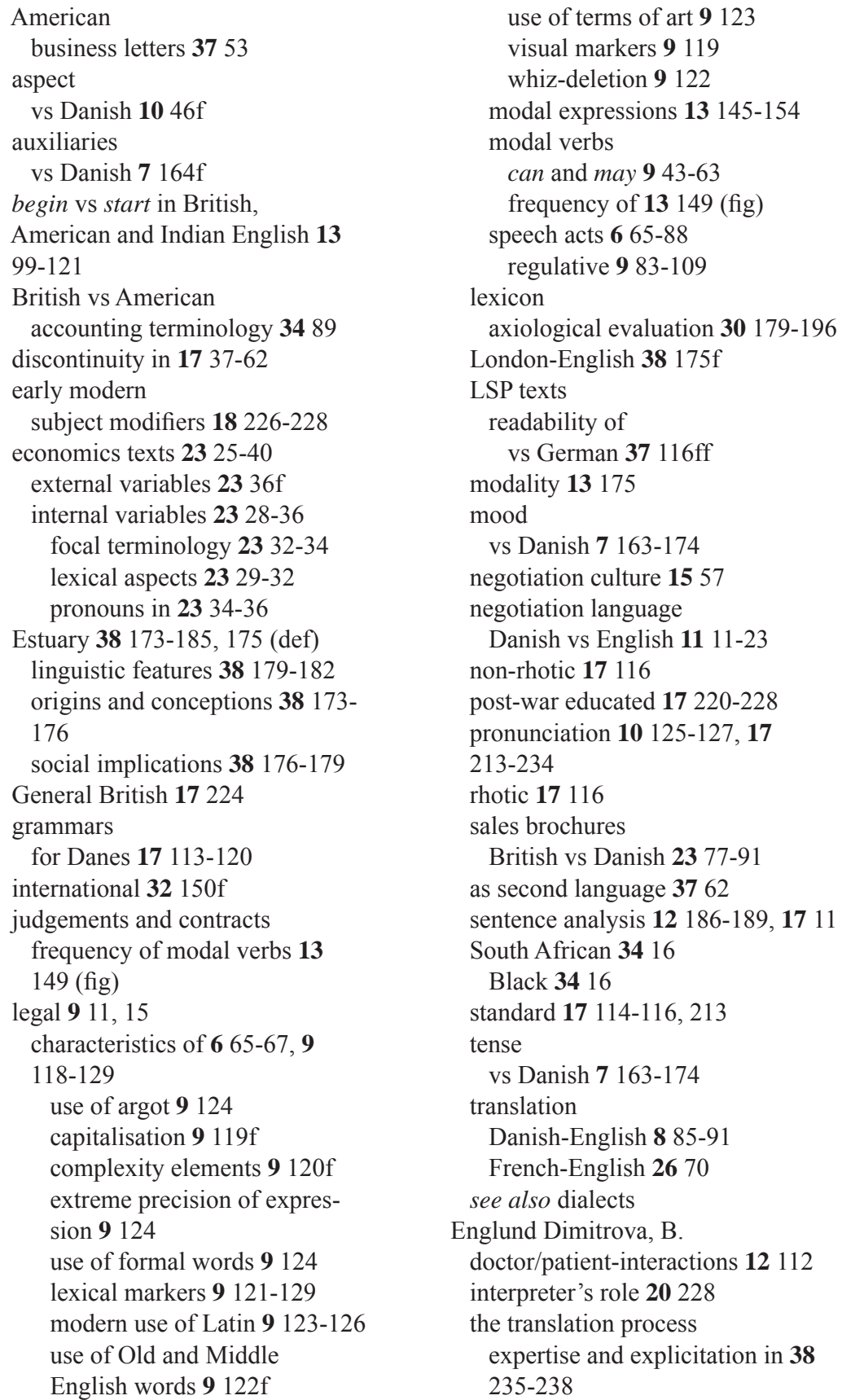


enlightenment

ancient Greece 377

classical European 377

post-modern 377

entities

entity types 2597 (fig)

entitlement 37 17-19, 18 (def)

ENTREVIS 7 81-84, 1310

EOIR $12132 \mathrm{f}$

EOSU 772

Eppler, Martin J.

black box 3713

knowledge communication 3712

equivalence 12 14f, 1457 (def), 36 205

adressing 28 203-206

approximative $\mathbf{1 2} 12$

bilingual dictionaries 28 195-208

communicative 14 57-60

concept of $\mathbf{1 2} 11$

criticism of $1213 \mathrm{f}$

dynamic 12 12, 14

experiments 1459

facultative 1212

full 28 196f

functional 1447

in LSP texts $1214 \mathrm{f}$

notion of $\mathbf{1 2}$ 12f, 17245

partial 28 197-199

postulate 1272

structural 1212

surrogate 28 199-202

total 1212

zero 28 199-202

Erickson, Bonnie

powerless language 36132

Eriksson, Anders

prosody 3324

error signals 638

ESIT 1276

Eskimo-Aleut language family $\mathbf{3 5} 59$

ESL $\rightarrow$ English

Esselink, Bert

desktop publishing 36213

internationalization 36211 localisation 32 191, 36 197ff, 209

(def)

software engineering 36212

terminology $36214 \mathrm{f}$

transcreation 36210

translation 36 197ff

software $36211 \mathrm{f}$

technology 36213

Estuary English 38 173-185, 175 (def) linguistic features 38 179-182

origins and conceptions 38 173-176

social implications 38 176-179

ethics $3892 \mathrm{f}$

ethos 37 49f

$\mathrm{EU}$

do EU-countries like each other?

$1568 \mathrm{f}$

importance of culture in the EU

and NAFTA areas 15 55-60

translations 32 195-198, 34 293-297

Europe

languages of 35 54-61

European functionalism 38196

europeanisms 35 45-71

Even-Zohar, I.

translation 1271

event 17 80-83, 80 (def)

evidentiality

Danish 31 236-239

EVS $\rightarrow$ ear-voice span

exercitives 672 (def)

experts 18102 (def)

explicatures 28 128-131

expressions

categorizing 17279 (def)

circumstantial 17279 (def)

collocationally closed $1322 \mathrm{f}$

collocationally open $\mathbf{1 3} 22 \mathrm{f}$

contained in enumerations 17279

(def)

of contrast to the main theme $\mathbf{1 7}$

278 (def)

empty sense 1327,28 (fig)

evaluative 17279 (def)

explicitations 17278 (def) 
frequencies of variations 13 33-35 fully compositional $\mathbf{1 3} 21$

metatextual 17279 (def)

modal 17279 (def)

non-compositional 1322

partly-compositional 1322

polysemous vs monosemous 13

26, 27 (fig)

pseudo-compositional 1321

received 1319 def, 20ff, 36-40

initial determiners in $\mathbf{1 3} 39$ (fig)

reformulations 17278 (def)

repetitions 17278 (def)

syntactic functions of $\mathbf{1 3} 35 \mathrm{f}$

syntactic structures of $\mathbf{1 3} 31$ (fig),

31f, 32 (fig)

see also collocability; formulaicity

Extended Standard Theory 16195

extracts 25121

extraposition 16187 (fig)

$\mathbf{F}$

F0

and prosodic phrasing 13 205-212

Fabbro, F.

simultaneous interpreting

neurolinguistic research in $\mathbf{2 2}$ $161 \mathrm{f}$

Faber, Pamela

axiological evaluation 30195

lexical structure and cognition $\mathbf{3 0}$ 180

face 12 146, 2776

-flattering 2777

-needs 3 255-257, 12 146-154, 15

61f

Spanish 31 126f

-saving 687

-threatening 6 75, 2776

-work 3 255-257

vs politeness strategies 2776

affiliation 3 255-257

autonomy 3 255-257 esteem 3 255-257

interpersonal 3255 (def), 12146

intrapersonal 3255 (def)

negative 6 75, 985 (def)

personal 12146

want 2776

face-to-face interpreting $\rightarrow$ inter-

preting (dialogue)

Færch, Claus

aspect in English 1476

communication problems 1592

communication strategy 3769 (def)

failure triggers $\mathbf{2 3} 158$

Fairclough, N.

genre 1653

Falk, J.

duetting 1121

false friends

phraseological 35 66-68

Fant, Lars M.

Hispanic-Scandinavian interaction 1517

negotiations 1110

Fauconnier, G.

conceptual blending theory $24138 \mathrm{f}$

conceptual integration 24140

mental spaces 38229

FBI

interpreter training $\mathbf{1 2} 133$

Feak, C.

academic writing

English 2524

Western academia 2543

plagiarism 28 51f

Felber, Helmut

CTN 1815

Felder, Ekkehard

legal communication 3611

Fenton, Sabine

court interpreting $20224 \mathrm{f}$

Ferrara, K.

blurred genres 2395

Fidelitätsregel 16 206, 214, 216

figure (Goffman's) 12177

FIKON 12 109, 112f, 123 


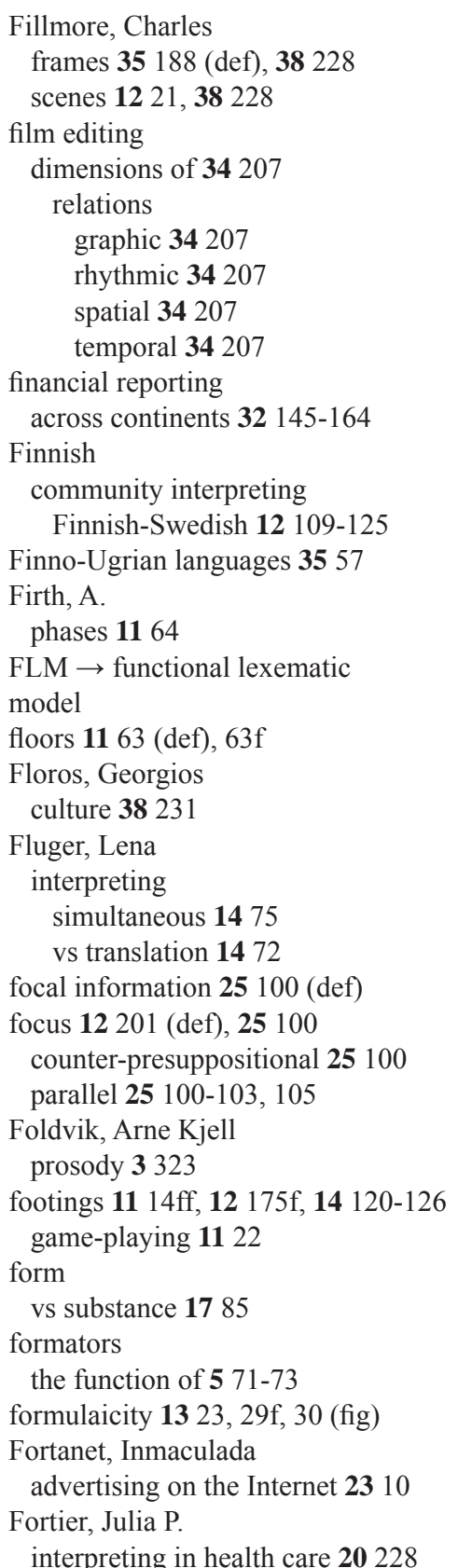

Foster, Don

gender-based language differences 36153

Foulkes, Paul Estuary English 38175

Fox, R.

text corpora for LSP 2527

Frade, Celina legislative writing 3210

frames 12 21f, 2596 (def), 28 117, 34206 (def), 35188 (def), 38228

(def), $228 \mathrm{f}$

filmic 34206

recruitment of $\mathbf{3 4} 218$ (fig)

the game frame $\mathbf{1 1} 22$

Francis, D.W.

teamwork 1173

Frandsen, Finn

business rhetorics 278

communication in public $388 \mathrm{f}$

Fraser, Bruce

metaphors $20165 \mathrm{f}$

free combinations 7131 (def), 135

French

academic writing 25 23-44

adjectives

in LSP texts 37154

anglicisms in 22 198-203

antipassive

reflexive verbs 19 75-90

and Chinese at the utterance level

13 351-353

clitics in 19 101, 103f, 110, 20

126f, 130-133

company brochures

argumentative strategies 19

297-301

vs Danish 25 217-226

connectors

tandis que 25 93-109

LGP dictionaries

loan words in 37155

neologisms in 22 198-203

reflexive verbs

antipassive in 19 75-90 


\author{
functional description of 19 75-90 \\ requests \\ vs Dutch 1562 \\ translation French-English $26 \mathbf{7 0}$ \\ Fretheim, Torsten \\ prosody 3323 \\ Friulian \\ clitics in 19100 \\ Funayama, Chuta \\ simultaneous interpreting 14138 \\ functional grammar 38196 \\ in Denmark 6 181-183 \\ of discourse 25 94-98 \\ English 20 272-277 \\ European functionalism 38196 \\ 4th International Conference on $\mathbf{6}$ \\ 181-183 \\ lexicon 30179 \\ functional lexematic model 30180 \\ functionalism 16 179f \\ fuzziness $\mathbf{3 2} 69$ \\ fuzzy sets 3264
}

G

Gårding, Eva prosody 3324

Gadamer, Hans-Georg reality of language 24121

Galician

clitics in 19 98, 103

Gallina, S.

linguistics, discourse analysis and interpretation 22165

Gambier, Yves

interpreting 22 160-167

Gamero, Silvia

translation 32172

Garber, Nathan interpreting $20222 \mathrm{f}$

Garfinkel, H.

connectors $\mathbf{5} 82$

Garnett, James L.

public communication 3867

public services loss of credibility 3867

Garrido, Joaquín

text cohesion 13331

Gaudin, François

militant lexicography 37155

Gazdar, Gerald

linguistics 16200

Geeraerts, D.

cognitive semantics 1857

Geluykens, Roland

listing 25102

gender 12 53-62, 36131

-switch 1255

in courtroom language 36 129-154

grammatical $1253-56$

pronominal 1253

significance for language use $\mathbf{3 6}$

$148-150$

social 12 56-62

in translation 12 51-62

gender systems

natural 1253

pronominal 1253

General British 17224

generality $3267 f$

Generalized Phrase Structure

Grammar 16194

generative grammar 6 181f, 16185

the data-base of a $820 \mathrm{f}$

generic structure potential 1644

genetic engineering $3725 \mathrm{ff}$

food containing GMOs $3725 \mathrm{ff}$

in the European Union 3726

in the U.S. 3726

genetically modified wheat 37 23-43

regulating the release of 37 29-31

Geneva School

dialogues 3846

genre 19214 (def), 231 (fig), 2314

(def), 15 (def), 93-101, 25 133-140,

3132 (def), 3716 (def), 50 (def)

academic introductions 19230 (fig)

blurred genres $\mathbf{2 3} 95$

categorising 3279

classification 23 13-23 
communicative purpose 23 13-23, 25 77-81

company brochures 27216 (def)

Danish and German brewery

brochures 27 215-227

a functional interpretation of $\mathbf{2 1}$ 199-202

comparison $3172 \mathrm{f}$

concept 28 116-119

construction of 31 31-43

context

-sender/receiver $22115 f$

receivers

direct 22115

indirect 22115

senders 22115

direct 22115

indirect 22115

as conventionalized communicative event 16 46f

cultural expectations related to genres 37 47-63

determination 23 13-23

hierarchy 19234

Internet $\mathbf{2 3} 95$

characteristics of 23 96-99

investigation 19236

and language learning $19237 \mathrm{f}$

legal 32 29ff, 78

binomial and multinomial structures 32 59-62

legislative $\rightarrow$ legal

models

pragmatic 25 77-81

move-structure 19 211, 22114 ,

116-119, 27 65-69, 74f, 31 39-41

at macro-level 22 116-118

moves 19 215, $236 \mathrm{f}$

at macro-level 22116

at micro-level 22116

primary vs secondary 2395

profiles 27 220-223

re-defining 2394

vs register $\mathbf{1 6} 45$

schematic structure $2314 \mathrm{f}$ service information pamphlets communicative purpose $3138 \mathrm{f}$

Danish 31 31-43

move-structure 31 39-41

rhetorical strategies 3141

as a staged, goal oriented social

process 16 43-46

step-structure 22 116-119

at micro-level $22118 \mathrm{f}$

steps

at macro-level 22116

at micro-level 22116

strategies $19236 \mathrm{f}$

technical $2370 \mathrm{f}$

theory 16 39-57

thick descriptions 19 220-222

as typified rhetorical action $\mathbf{1 6}$

41-43

versatility in $19228 \mathrm{f}$

genre analysis 19 207-213, 21 199,

27218

application of $22131 \mathrm{f}$

of legal discourse 22 113-133

methodological issues in $\mathbf{1 6} 39-57$

a model for 22 113-115

of multiple texts $3168 \mathrm{f}$

the use of specialist informants in 19 223-226, 235

Genteknologisk ordbog dansk-

engelsk/engelsk-dansk 10121

Gentile, Adolfo

community interpreting 20 215f, 222

Gentzler, Edward

translation 31207

German

academic writing

criticism in $\mathbf{2 5} 25$

articles about business cycles metaphors in 24 57-79

brewery brochures 27 215-227

Chinese intermediate learners unsatisfactory dictionary situation $30216 \mathrm{ff}$

court decisions 36 15-39 
language needs in Danish companies 31 13-28

legal

modal expressions 13 145-154

modal verbs

frequency of 13150 (fig)

LSP texts

readability of

vs English 37 116ff

modality 13175

negotiation culture 1557

Germanic languages 3556

Gerzymisch-Arbogast, $\mathrm{H}$.

translation

and knowledge systems $38221 \mathrm{ff}$

and text $\mathbf{2 6} 12$

Ghazi, Joseph

combining forms vs affixes 25

17-19

Gibbs, Raymond

metaphors 35188

Gibson, James J.

affordance 2427

Gibson, T.R.

abstracting 25125

Gibson, William

Neuromancer 24 19f

Gilbert, Judy B.

teaching grammar $\mathbf{3 1} 98 \mathrm{f}$

Gile, Daniel

classification of information

sources 32161 (fig)

effort models 1279

interpreting 22 160-167

literature on 1281

methodology $22163 \mathrm{f}$

the role of knowledge in $\mathbf{3 8} 227$

interpreting research 1278

developments 1411

history of $\mathbf{1 2 7 4}$

policy 22163

memory overload 23159

practisearchers 1282

problem triggers 23157 scientific vs unscientific research

1268 (def)

translation 32146

dominance of linguistics 1283

and text $\mathbf{2 6} 13$

translator qualities 32160

Gillaerts, Paul

job applications 3112

Gilsdorf, Jeanette

comma splice 3194

teaching grammar 31 96, 99

Gimson, A.C.

pronunciation of English 17232

RP 17233

revised $17223 \mathrm{f}$

standard English 17218

Giraldo, J.J.

translating medical texts $\mathbf{2 5} 9$

glossematics 6181

Göpferich, Susanne

specialized knowledge $37 \mathrm{7f}$

Goffman, Erwing

communication 3270

the concept of territory $\mathbf{2 7} 77$

dialogues $\mathbf{3 8} 45$

face 31126

facework 2776

interpreting 14121

negative dialogues 2179

participation status 1114

role theory 12174

Goméz, Miguel Casas

verbs 6187

Gorcy, G.

polysemy in dictionaries 8105

Gorter, $\mathrm{T}$.

Dutch and French cultural beha-

viour 15 60f

Gottlieb, Henrik

English-Danish idiomatic dictio-

nary 12211

etymological information in tradi-

tional dictionaries 12212

screen translation 17 244-250

technical texts $\mathbf{2 3} 68$ 
Gouws, Liezl

Nuwe Woordeboek sonder grense 38 217-220

Gouws, Rufus H.

learner's dictionaries irregular flexion variants in $\mathbf{3 8} 219$

Nuwe Woordeboek sonder grense 38 217-220

Government and Binding 16 195, 196-198

GPA 29 316-318, 317 (fig)

Graber, Doris A.

communication 3867

Graham, John L.

negotiation 3 203, 15 13, 18

intra-cultural 1513

problems in $\mathbf{1 5} 14$

grammar 3125 (def), 17 97-101

Collins/Cobuild 17114

communicative

Spanish 12 189-192

as construction kit 1768 (def), 68f

English

for Danes 17 113-120

functional $20272-277$

functional 38196

in Denmark 6 181-183

of discourse 25 94-98

English 20 272-277

European functionalism 38196

4th International Conference on

6 181-183

lexicon 30179

generative 6 181f, 16185

the data-base of a $820 \mathrm{f}$

grammars 38 119-122

vs dictionaries 38 119f

grammatical description 17 75-101

hyper-text based 17 63-72

learning with $1769 \mathrm{f}$

Inter- 12 141-156

interpretively oriented $\mathbf{1 7} 76$

linguistic functions and super-

types 17 83-86

mental 17101 in modern curricula 31 84-86

multifunctional 17 75-101

Russian 17 75-78

single source 1770

squinting 1975

teaching

selectively 31 83-100

TG- 10 11-15, 19

transformational 1211

universal 8 20, 17 77f

university

for foreign students $\mathbf{1 7} 120$

valency $38196 f$

see also case theory; descripti-

vism; Generalized Phrase

Structure Grammar; glossematics;

Government and Binding; Head

Driven Phrase Structure Grammar; prescriptivism; Standard

Theory; Theory of Binding and

Control; Trace Theory; WebCT;

X-bar-theory

grammatical categories

number 1796

semantics of 19 241-251

grammaticality 8 7-21

degrees of 8 18-20

grammatical filter 813

grammaticalization 38 190-192

in Spanish 38 192-194

Gran, L.

simultaneous interpreting neurolinguistic research in 22 $161 \mathrm{f}$

Granacher, Martin

failure triggers 23158

Granström, Björn

prosody $3323 \mathrm{f}$

graphs

bi- $13187 \mathrm{ff}$

tri- $13187 \mathrm{ff}$

Great Chain of Being 30184

Green Globe, The 2762

green hotels

brochures 27 64-73 
the rhetoric of 27 69-73

in Denmark 27 55f, 62-64

green cards (towel cards) 27 73-80

linguistic politeness in model for the analysis of $\mathbf{2 7}$ 78 (fig)

the rhetoric of 27 75-80

Green Key, The 27 62, 64

Greenbaum, Sidney

connectors 577

Greenberg, J.H.

case system 1993

Gregersen, Frans

urban sociolinguistics 9 211-216

Grice, H.P.

communication 695

maxim of quantity $\mathbf{3 1} 199$

maxims of conversation 5 40ff,

64, 78

Gringiani, A.

interpretation research and

training 22163

Groeben, Norbert

comprehensibility of texts $\mathbf{3 6} 25$

Grøn, Hanne

legal translation 9 16f

Grønnum, Nina

prosody $3322 \mathrm{f}$

group orientation

vs individualism 15 56f

Grubitzsch, Helga

Les Mystères de Paris 1238

Grundy, Peter

cultural expectations of business letters 3753

Grunig, J.

asymmetrical dialogues $\mathbf{3 8} 45$

dialogues 3844

GSP $\rightarrow$ generic structure

potential

Guattari, Félix

transversality $\mathbf{2 4} 100$

Gubba, Wilhelm

LSP dictionaries 3 133ff

Gudykunst, W.B. communication between strangers

31124

interaction with strangers 31 123f

Guilbert, Louis

neo-classical compounds 2515

special vocabulary 1817

Gumperz, J.

contextualisation cues 2860 (def)

Gunnarsson, B.-L.

development of economics

writing $19253 \mathrm{f}$

gender-based language differen-

ces 36153

Gustafson, Kjell

prosody $3323 \mathrm{f}$

Gutt, Ernst-August

translation $\mathbf{3 1} 206$

\section{$\mathbf{H}$}

Habermas, Jürgen

speech situations 3742

HABLASAL 1310

Hagendoorn, L.

EU-countries 15 68f

Hagiwara, Naoyuki

simultaneous interpreting 14137

Hahn, Walter von

knowledge in MT systems $\mathbf{3 8} 223 \mathrm{f}$

Hale, S.B.

court interpreting 20227

Hall, B.

interaction with strangers 31 123f

Hall, Karin R.

translation 23207

Hall, S.

silences in communication $\mathbf{2 8} 81$

Halliday, M.A.K.

equi vs skewed probability hypo-

thesis $\mathbf{1 3} 104$

general nouns 2878

language functions 1783

stratificational model of dialogue

28114 
Hammond, N.

computer-based learning materials 17 69f

Hansen, E. administrative language $\mathbf{3 1} 35$

Hara, Fujiko socio-cultural problems of Japanese 14140

Harder, Peter aspect in English 1476 diachronic and synchronic analysis $\mathbf{3 8} 187$

functional grammar 6182 generative grammar and glossematics 6181

Harmaakivi, Carola equivalence 1459

Harrap's Essential English Dictionary

biotechnological terms in monolingual dictionaries 18 103-107

Harré, R.

greenspeak 2758 (def)

Harris, Brian

interpreting 20 223f, 2556 vs translation 1474

translational norms 12 83-85, 14 66,68

Harris, Z.

multilayered structures $\mathbf{1 0} 11$

Harrit, Jørgen

Danish-Russian dictionary 12214

Harry Potter 32 186f

Hartmann, R.R.K.

defining macrostructure 450 problem of equivalence 1211

Hartvigson, Hans

English grammar 1711

Hasan, R.

general nouns $\mathbf{2 8} 78$

genre 1653

genre theory $1643 \mathrm{f}$

Hashim, Azirah

the Malaysian arbitration act 3210
Haslev, Marianne

prosody 3323

Hatim, Basil

equivalence 1214

literature vs non-literature 2372

scientific and technical communi-

cation 2373

text types 2374

translation 21 227, 2617

Hauge, Hans

G. Lakoff and M. Johnson 2410

Hausmann, Franz Josef

classification of dictionaries 6 92-94

defining macrostructure 451

Have, Paul ten interviews 32 119f, 132, 134

Haverkate, Henk

pragma-linguistic approaches $\mathbf{1 3}$

$331 \mathrm{f}$

Head Driven Phrase Structure

Grammar 16194

head-final languages 1023

head-first languages 1023

hearer-based languages 17 84f

Hearit, Keith M.

apologies 38 86ff

Hecht, Christian

prosody 3324

hedges 36136 (def), 137-139

in courtroom discourse 36136

Heinemann, Trine

negative interrogatives $\mathbf{3 8} 77,80$

requests $\mathbf{3 8} 71$

interrogative 3872

Hellinger, $\mathrm{M}$.

social gender $1257 \mathrm{f}$

Helsinki Corpus of Early English

Correspondence 13 135-142

problems of authenticity 13 137-139

problems of coverage 13 139-141

social representativeness $13140 \mathrm{f}$

Heltoft, Lars

Danish

word order 12 199, 202f

topological approach 12201 
vs H. Korzen 12206

topological phenomena

and generative grammar 12200

Hengeveld, Kees

pragma-linguistic approaches 13

$331 f$

Henry, F.

computerization of dictionaries 8

$108 \mathrm{f}$

Herberger, R.A.

negotiations 3203

Herbert, Jean

interpreting 1274

theory of sense 1277

Herbst, T.

grammars vs dictionaries 38 119f

Hermans, Theo

linguistics in literary translation studies $\mathbf{1 2} 71$

translational norms 12 73, 1467

Hermes 6 7, 337

changing language policy 359,367

new subtitle 359

Hernández Flores, N. confianza 31 127f

Herndl, C.G.

environmental discourse 2758

Herslund, Michael

the Adject Theory 38196

basic textual mechanism $1612 \mathrm{f}$

bilingual lexicography 8103

modality and the indefinite article 31 234-236

relation between verb and fundamental argument 38198

word order 12 198ff

High Court 3287 (def)

in the Malaysian Arbitration Act 3287

Hill, Forbes I.

rhetorics $3748 \mathrm{f}$

Hinkel, E.

plagiarism 28 13, 15

Hjelmslev, Louis

inductivism 2182
Hjørnager Pedersen, V.

Dansk-engelsk ordbog (V/B) 12213

translation 1238

Høeg-Müller, Henrik

modality 31 229-239

Spanish modal auxiliaries 31

231-234

Hönig, Hans G.

translation 12 13, 52

micro-strategies 2457

recipient roles 23 207, 209

Høy, Asta

medical terms 258

Hoey, Michael

the Automatic Abridgement

Project 778

Hoffman, D.L.

banner ad 23101 (def)

Hoffmann, Lothar

structural matrix/text-external

matrix 27218

Hofstede, Geert

cross-cultural discourse 3763

culture 15 54ff

dimensions of 3711

impact on international business

success 1554

holems 38231

Holmes, James S.

translation $\mathbf{3 1} 202 \mathrm{f}$

normativity 12172

scientific approach 12169

studies 1270 (def)

descriptive 1266

Holmes, M.E.

phase structures in negotiation $\mathbf{1 1} 64$

holons 38231

homo transferens 1269

Hopper, Paul J.

grammaticalization 38191

markedness 38190

House, David

prosody 3324

House, Juliane

complaints 2172 
speech acts 1534

translation 12 68, 16 204f, 207.

213, $217 \mathrm{f}$

Houtkoop-Steenstra, H.

interviews 32 119, 131f

Howard, R.M.

patchwriting 2840

HTML 2489

Huckin, Thomas N. genre 16 49f, 54, 23 94, 3750 (def)

definition of 2396

expectations 3752

genre and text analysis $\mathbf{1 6} 42$

silences in communication $\mathbf{2 8} 82$

textual silence $\mathbf{2 8} 83$ (def)

Hulst, Jacqueline

translation 1214

Humbley, John

neology 37155

purism 8109

Hume, David

the doctrine of induction 2182

Hunston, S.

English academic writing 2524

Hunt, T.

asymmetrical dialogues $\mathbf{3 8} 45$

dialogues 3844

Hurtado Albir, A.

translation errors 2619

Hutchby, Ian

interview data 27 178-180

semi-structured interviews 32121

Hymes, Dell

discourse events 2594 (def)

Hyper-Text Markup Language

$\rightarrow$ HTML

hypertext 17 63f, 2398

hypertextuality 24 81-105

the term 2489

\section{I}

I-language 8 8ff, 38223

IAS 32 145ff, $38205 f$

IC-analysis 109
ICAME 139

icons 586

diagrammatic 586

image 586

metaphorical 587

ICSB

36th World Congress 1441

idioculture 1440

idiom research 35 45-49

and language families $3554 \mathrm{f}$

idioms 7 130f

European languages 35 50-53,

62-65

cross-linguistic uniformity 35

45-71

metaphorical 35187

origin of 35 68-70

the term 3546

types of 35 50-53

widespread 35 50, 62-65

see also collocations; euro-

peanisms; free combinations; interphraseologisms; internatio-

nalisms; phraseologisms; phrases

IDS Mannheim 3457

IFRS $38205 f$

Iguchi, Sanae

simultaneous interpreting 14136

Iivonen, Antti

prosody 3323

illocutions 15 45f

image 2760 (def)

restoration strategies $\mathbf{3 8} 87-89$

images 24 92f

scriptualization of 24 94-100

visual 24 138-144

implicatures 28 128-131

individualism

vs group orientation $1556 \mathrm{f}$

Indo-European language family 35

55-57

Indo-Iranian languages 3557

induction $\rightarrow$ inductivism

inductivism 2 179-185

inferencing $\mathbf{3 8} 232$ 
infinitive 19105

information 696

vs knowledge 38 226, 233

packaging 3256

structure $12201 \mathrm{f}$

information bank 1768

information costs

lexicographical $3487 \mathrm{f}$

comprehension-related $3487 \mathrm{f}$

search-related 3487

Information Highway 24 133-141,

156

information management $\mathbf{1 7} 70 \mathrm{f}$

information retrieval

in bilingual dictionaries 28 202-208

information sources

classification 32161 (fig)

information technology $\rightarrow$ IT

Ingarden, $\mathrm{R}$.

meaning 571

Inglewood, Eric

The City of Angels 3169

integrativeness $\mathbf{3} 254$

intelligence

human vs machine 25 113-129

inter-phraseologisms 3548

interaction 12 142, 24 81-105, 3846

between strangers 31 119-133

and communication 17 67f

courtroom 32241

cross-cultural 1582

business

handling of complaints in $\mathbf{2 1}$ 67-91

everyday

handling of complaints in $\mathbf{2 1}$ 67-91

face-to-face 12149

interpreting as 14 111-128

question-response 32 131-137

strategies

modulating 12147

interpersonal involvement 12

154f

positive/negative affect $12153 \mathrm{f}$ trimodal 12 146-151

verbal and nonverbal 12 141-156

interactional aims 6 19ff

interactive behaviour $\mathbf{1 3} 66$

intercultural layer 11 19f

Intergrammar 12 141-156

intergroup layer 11 16-18

interlanguage layer 11 20-22

interlingual transfers $844 \mathrm{f}$

Internalized language $\rightarrow$ I-language

International Accounting Standards

32 145ff

internationalisms 35 45-71

internationalization 36211

Internet 23 94-101

-politics $24131 \mathrm{f}$

advertising on the 23 93-110

genres 2395

characteristics of 23 96-99

identity in the 24 84-86

ideology of the 24 151-157

as information highway 24 133-141

a media-philosophical analysis of

the 24 81-105

space and time in the $\mathbf{2 4} 86 \mathrm{f}$

see also World Wide Web

Internet Relay Chat 24 84-93

interpersonal layer 11 15f

interphraseologisms 3548

interpreting 845 (def)

ad hoc 20213

Bilingual Skills Certificate 12134

bilingualism

research on 15 143-152

business 20214

as communication 12 36-41

community $12113,124 \mathrm{f}, 128$

(def), 135f, 14 115, 20 214-217,

$230 \mathrm{f}$

Certificate in Community Inter-

pretation 12134

vs conference 12128

Finnish-Swedish 12 109-125

non- 20216 
training

in the United Kingdom 12

133-135

in the United States 12 129-133

in community organizations

and academic institutions

12 129-132

in government agencies $\mathbf{1 2}$ $132 \mathrm{f}$

conference 12 113, 14 15, 20

212f, $230 \mathrm{f}$

current trends in research 22

160-167

non- 20 212-217

research on $20217 \mathrm{f}$

consecutive 8 45, 12 113, $17246 f$

text condensation in $\mathbf{1 7}$ 273-281

court 12127

pragmatic meaning 32 237-243

training 12127

courtroom interaction 32241

criminal proceedings ind Danish

district courts 32 237-243

cultural 20215

as cultural transfer $\mathbf{1 4} 34,40$

and culture 14 139f

deletions in 17 277-280

semantic-pragmatic analysis of 17278

syntactic analysis of $\mathbf{1 7} 278$

dialogism $14113 \mathrm{f}$

dialogue 14 111-128, 20 213, 230

dilemma 14126

in immigration hearings and

medical encounters 12 167-181

direction of 15 150-152

errors and omissions in 17 277-

280, 22 51-68

EU- 20213

fusion $14137 \mathrm{f}$

in health settings 20 211-231

humour 14 44-48

as interaction 12 167-181, 14

111-128

interdisciplinarity 14 17-19 1th International Conference on

20 211-231

the interpreters' notes $\mathbf{3 8} 229 \mathrm{f}$

intratextual coherence $1438 \mathrm{f}$

knowledge-related aspects of $\mathbf{3 8}$

221-234

in legal settings 20 211-231

in Denmark $20216 \mathrm{f}$

legislative $3249 \mathrm{f}$

liaison 20213

linguistic competence 14 57f, 60f

linguistic performance 14 57f, $61 \mathrm{f}$

London Interpreting Project 12135

monologism $14113 \mathrm{f}$

omissions 26 53-57

paradigms

two groups of interpreters 12

76, $1416 f$

liberal arts community $\mathbf{1 2} 76 \mathrm{f}$

natural science community $\mathbf{1 2}$

78-80, 14 16f

participation framework 12 175,

14 120-127

performance, variability of 26 47-53

performances 1462

oral vs written $\mathbf{1 4} 74$

problem triggers $23157 \mathrm{f}$

production formats 12 176, 14

121f, 127

question-answer dialogs 32 237-243

reception formats 12 176, 14 121,

127

reference identification in 12 29-49

relaying by displaying $12177 \mathrm{f}$

relaying by replaying $\mathbf{1 2} 178$

role theory 12174

simultaneous 8 45, 12 113, 14

132f, 15 150, 17246 (def), 23

153-169

Dynamic Model 14139

effort model on 12 79f

Finland 1455

functionalist perspective 14 31-50

methodological and didactic

reflections 10 41-50 
perception of fidelity in 22 51-68 difficulty of fidelity assessment 22 51f

fidelity ratings $2262 \mathrm{ff}$

progress 14 55-62

of read-out speeches 26 41-61 research 15143

neurolinguistic 22 161f

simultaneity 17247

skills 10 41-50

skopos in 14 35-38

translational norms 14 65-85

working memory 14134

skills $12116 \mathrm{f}$

social role of the interpreter $14115 f$

in social service settings 20 211-231

speaker-hearer roles 14 120-123,

126

study of

relevance-theoretic approach $\mathbf{3 8}$ $232 \mathrm{f}$

theoretical base of 14 31-50

theory-forming work 1465

three-cornered interpreting 20213

timelag 26 53-57

tourist 20214

vs translation 8 43-53, 10 43f, 14

31-50, 31ff, $72 \mathrm{f}$

translational norms 14 65-85

transmission conditions 10 44f

the 1994 Turku Conference on

Interpreting 22 160-167

see also dubbing; effort models;

failure triggers; problem triggers;

simultaneity; subtitling; translation; voice-over

interpreting research 12 65-86, 167 -

170, 14 11-13, 15-27, 113, 15 143,

20 211f, 26 40, 97-115

contemporary perspective 12 76-82

historical perspective 12 74-76

in Japan 14 131-141

Manipulation School of translation

studies 12 65-86

neurolinguistic 22 161f by non-interpreters 1422

obstacles 14 19-21

Paris School 1276

product-oriented 14 31-50

schools $1421 \mathrm{f}$

in Sweden 12 111f

text-oriented 26 39-61

translational norms 12 83-85, 14

114-116

concept of 14 66-69

initial norms 1467

operational norms 1467

preliminary norms 1467

usefulness of 26 98-102

Interpreting Research Association

of Japan 14131

interrogative requests $\mathbf{3 8} 72$

interrogatives

negative 38 71, 77f, 80

intertextuality $3265 \mathrm{f}$

legislative $3265 \mathrm{f}$

practices 28 39-56

interviews 27 173-191, 32 117-142

the conversational dilemma 32120

interactive phenomena in the dis-

cursive construction 27 173-191

methodology 27 173-191

question delivery structure $32132 \mathrm{f}$

response delivery 32 133-137

research- 32 117-142

the discoursive organization of

27 173-191

interactive resources 32 117-142

the research interview as a

dialogue 27173

semi-structured 32 121, 125

vs structured $32117 \mathrm{f}$

true and valid responses 32

$118-120$

verification 32 137-141

intonation $\mathbf{1 3} 206$

groups $\rightarrow$ tone units

units $\rightarrow$ tone units

intratextuality $\mathbf{3 2} 65$

introspection 852 
"involvement first" principle 15 115, 124

IRC $\rightarrow$ Internet Relay Chat irony 5 49, 56

ironical utterances $552 \mathrm{f}$

Isenberg, $\mathrm{H}$.

interactional aims 619

Isham, B.

interpretation methodology 22164

ISO certifications 16 209-212

isotopy 21229

IT

commercializing the desktop 24

144-151

computer metaphors 24146

conceptual mapping of the desk-

top metaphor $\mathbf{2 4} 145$

ideology of information technology 24 131-158

interface as desktop metaphor $\mathbf{2 4}$

145

see also computer

Italian

clitics in 19 99, 101, 20 126f,

131-134

\section{J}

Jackson, Howard grammars vs dictionaries $\mathbf{3 8} 120$ grammatical information in dictionaries 3126

Jakobsen, Arnt L.

expert translators' processing

knowledge $38227 \mathrm{f}$

translation

LSP texts 10 115-123

model $10116 \mathrm{f}$

Jakobsen, Leif K.

English sentence analysis $\mathbf{1 7} 11$

Jakobsen, Lisbeth Falster

functional grammar $6182 \mathrm{f}$

Jakobson, Roman

gender problems in translation 12 55 language functions 1783

subject vs predicate 1723

Jamieson, Kathleen M.

genre $\mathbf{1 6} 48$

Janney, Richard W.

the computer as prosthesis 2418

Intergrammar 12 141-156

Jante, Law of 27224

janteloven 27224

Japan

business letters 37 53, 57f, $60 \mathrm{f}$

communication style 14140

interpreting research in 14 131-141

socio-cultural problems of Japa-

nese 14140

Jarvad, Pia

The Danish Language Council 8

106

neo-classical compounds 2516

Jefferson, G.

interactions 3846

Jensen, $\mathrm{K}$.

grammars 1764

Jiménez Hurtado, C.

superstructure of texts $\mathbf{3 2} 169$

job application letters $\rightarrow$ letters of

application

Jørgensen, Jacob

communication policies $3819 \mathrm{ff}$

Johansen, Winni

business rhetorics 278

communication in public $\mathbf{3 8} 8 \mathrm{f}$

Johns, Tim

EOSU 772

Johnson, Bruce

powerless language 36132

Johnson, Edward

PoliceSpeak 25118

Johnson, Mark

metaphor theory conceptual 24138

metaphors 18 81, 24 10, 35 185ff

vs metonymy 20177

Johnson, Ralph $\mathrm{H}$.

the relevance problem 5 57-59 
Jones, Bill

Margaret Thatcher 29314

Jones, Daniel

received pronunciation 17217

Jonsen, Albert

casuistry $\mathbf{3 8} 92$

Jordan, Birgitte

interviews $32118 \mathrm{f}$

the conversational dilemma 32 120

Josselin-Leray, A.

specialized entries in LGP dictionaries 37157

Jowitt's Dictionary of English Law macrostructure $458 \mathrm{f}$

junction $2670 \mathrm{f}$

Albert Camus 26 71-77

JUR-korpus 781

Juvonen, P.

repair in L2-instruction $\mathbf{1 5} 93 \mathrm{f}$

Jyske Bank

marketing communication $2745 \mathrm{f}$

sales policy 27 44-46

vs Spar Nord Bank $2750 f$

\section{K}

Kade, Otto

orality $\mathbf{3 1} 204$

Kahn, R.L.

organisations $3813 f$

Kalina, S.

interpretation methodology 22164

Kalverkämper, Hartwig

body language in business negotiations 2311

the influence of linguistic features

on a text 27217

specialist communication 287

user levels 6100

Kampf, Constance

culture

dimensions of 3711

as a system 3712

specialized knowledge 377
Kant, Immanuel

three kinds of reasoning 24117

Kaplan, Ron

lexicalism 16192

LFG 16194

using L2 in negotiations 1557

Kasper, Gabriele

communication problems 1592

communication strategy 3769 (def)

speech acts 1534

Kastberg, Peter

communication policies $\mathbf{3 8} 8$

culture

dimensions of 3711

as a system 3712

successful LSP texts 2310

Katamba, Francis

neo-classical compounds $2515 f$

Katan, D.

intercultural communication,

negotiation and interpreting 22165

interpretation research and trai-

ning 22163

kategoria 3890 (def)

Katz, D.

organisations $\mathbf{3 8} 13 \mathrm{f}$

Kaufmann, Uwe

a dictionary for gene technology

10121

Kavanagh, Dennis

Margaret Thatcher 29314

Kayser, Daniel

difference between words and terms 1823

Kazenin, Konstantin I.

semantic content of a verb 1990

KBMT 38224

Keenan, E.L.

Accessibility Hierarchy 821

Keerie, Natasha gender-based language differences 36153

in institutional discourse 36146

Keller, Kevin

communication policy $37102 \mathrm{f}$ 
Kenyon, Dorry M. translation skills 2617

Kerbrat-Orecchioni, C. critising $\mathrm{P}$. Brown and $\mathrm{S}$. Levinson 2776

face-flattering 2777

negative receiver-oriented politeness strategies 2779

Kerswill, Paul

Estuary English 38175

Kibbee, D.

translation 2667

Killie, Kristin early modern English subject modifiers 18 226-228

Killingsworth, $\mathrm{M}$. ecospeak 2758 (def)

Kim, Young-Jin communication between strangers 31124

cultural constellations in text and translation $38230 f$

Kiraly, Donald C.

translation 23207

Kirchhoff, Hella three-party two-language model 1044

Kjeldgaard, Dannie locality $36199 \mathrm{f}$

Kjellmer, G. corpus evidence 13104

Kleifgen, J.

communication 15 100f

Kloss, Heinz

terminology 1819

knowledge 37 7, 9-20, 38 221, 232

-creation 38226

-related aspects of translation $\mathbf{3 8}$ 221-234

communication

operationalizing 37 9-20

concept of 25 116-118

declarative $\mathbf{3 8} 222$

domain- $38224 \mathrm{f}$

vs information 38 226, 233 linguistic 38223

in machine translation systems 38

$223 f$

non-linguistic $38223 \mathrm{f}$

declarative $38223 \mathrm{f}$

procedural 38222,233

processing $38227 \mathrm{f}$

representation $\mathbf{3 8} 228$

specialized $3747 \mathrm{ff}$

mediation of 37 7f, 67-89

systems 34 199-221

tacit 25 113-129

and text types $38224 \mathrm{f}$

text- 38224

types $\mathbf{3 8} 221 \mathrm{f}$

world- $38224 \mathrm{f}$

knowledge systems

and translation 38 221-234

Knowledge Telling Model 35 190f

Knowledge Transforming Model

$35190 f$

Knowles, Gerry

definition of nucleus 13236

Kocourek, Rotislav

neo-classical compounds 2516

Kølsen de Wit, C.

business rhetorics 278

Kohärenzregel 16 206, 214

Kohno, Morio

simultaneous interpreting 14137

Koivuselkä-Sallinen, Päivi

prosody 3324

Kok, A. de

prosodic laws 20126

Kondo, Masaomi

intercultural communication, negotiation and interpreting 22165 interpretation and culture 14 139f interpretation research policy 22 163

Kopczynski, Andrzej deletion (interpreting) 17277 equivalence 1457 (def)

Korhonen, Jarmo

European idioms 35 47f 
Korzen, Hanne

vs L. Heltoft 12206

word order 12 203-206

Kotler, Philip communication policy $37102 \mathrm{f}$

Kotsinas, Ulla-Britt urban sociolinguistics 9 211-216

Kotter, John

change processes 38 54f, 58

Kourilova, M.

English academic writing 2524

Kragh, Bodil

LSP 8129

Krallmann, Dieter

international proverbial sayings 3548

Kramer, M.G.

plagiarism 2815

Kress, Gunther genre 16 44f, 50f

Kreuz, R.J.

miscommunication 15105

Kristensen, S.M.

primary and secondary public $\mathbf{1 2} 37$

Kristensen, Tine

localisation 32192

Kristiansen, Marianne dialogues 3845

Kristján Arnason prosody 3324

Kroes-Hecht, Maria prosody 3324

Kromann, Hans-Peder active-passive theory $\mathbf{3 1} 171$ Allbuch 6104

bilingual lexicography 8102 thesis about four dictionaries per language pair 6102

Kronning, $\mathrm{H}$.

auxiliary verbs 19 101f

infinitive 19105

Krzeszowski, Tomasz

linguistic semantics 30181

Kühn, Peter

dictionaries 698
Kume, Akimoto

consecutive interpreting 14132

Kundera, Milan

authentic spoken language 783

Kurz, I.

interpretation methodology $22163 \mathrm{f}$

Kurzon, Dennis

language of the law 912

Kussmaul, Paul

textual conventions $32171 \mathrm{f}$

textual knowledge $32181 \mathrm{f}$

translation 12 13, 52

Kuusi, Matti

3,000 variants of a saying 3546

the world history of a saying $3546 f$

Kvale, Steiner

interviews 32137

the conversational dilemma 32

120

research interviews 27175

KWIC-concordans 13 15f

Kytö, Merja

language interaction 36153

men's and women's language 36 147

L

L1 15144 (def)

and L2 15 144f, 148f, $150 f$

L2

research 15 148f, 150

Lachter, J.

TRICS 646

Ladkin, A.

the commercial green discourse

2762

Lag Sequential Analysis 1512

laísmo 38189

Lakoff, George

categorisation 1855

Great Chain of Being 30184

metaphors 18 81, 24 10, 35 185ff

vs metonymy 20177

theory $\mathbf{2 4} 138$ 
women's language 36130

Lakso, Tiina

errors and omissions in interpre-

tations 2253

Lamberger-Felber, Heike

effort models 23158

text and translation 2612

Lambert, José

translation criticism 1268

Lambert, S.

interpretation research policy 22

163

skill components in simultaneous interpreting 22164

Lamíquiz, Vidal

Spanish verbs 6186

Landau, Sidney I.

macrostructure 4 55-57

Langacker, Ronald W.

aspectual verbs

syntax and semantics of $\mathbf{1 3} 102 \mathrm{f}$

complementation $13120 f$

profiling 35188

valuation 30181

Langendoen, D.T.

acceptability and grammaticality 814

language 3 307f, $2492 f$

academic 28 7-9

acquisition 6 31-61, $19237 \mathrm{f}$

connectionists approaches to $\mathbf{6}$

31-61

learning the English past tense

6 42-48

overgeneralisations 6 42ff

the "poverty of the stimulus"

argument 634

administrative 31 31-43

and anthropology 38 107-109

attested 29286 (def)

attrition

research $15149 f$

temporary regression $15149 f$

children and 6 42-47, 53-59

common vs specific 6 105, 2566 core vs periphery $\mathbf{3 8} 190$

courtroom 36 129-154

and culture 12 19, 23 86-89

and dialect $\mathbf{3 6} 200$

differences

gender-based 36 129-154

E- 38223

Europe 35 54-61

families

and idiom research $3554 \mathrm{f}$

functions 17 83-86

appeal function 1783

expressive function 1783

representative function 1783

gender significance 36 148-150

head-final 1023

head-first 1023

hearer-based $1784 \mathrm{f}$

history 13 135-142

I- 8 8ff, 38223

interpretation 3 287-308

knowledge

general vs special 6104

the language game model 3271

langue/parole 38108

learning $\rightarrow$ acquisition

LGP vs LSP 6 105, 2566

mediator 1044

men's and women's 36 129ff

cultural approach to 36130

deficit approach to 36130

dynamic approach to 36131

natural language identification $\mathbf{1 3}$

183-203

methods for 13 185-188

planning 1819

in France $1821 \mathrm{f}$

in Québec 18 19-21

policy 37 95-113, 107 (def)

vs communication policy $37111 \mathrm{f}$

defining 37 106-108

general $37100 f$

interlingual 37107 (def)

general 37107 (def)

specific 37107 (def) 


\begin{tabular}{|c|c|}
\hline & $\begin{array}{l}\text { Latour, B. } \\
\text { science } 2523\end{array}$ \\
\hline $\begin{array}{l}\text { intralingual } 37108 \text { (def), } 112 \\
\text { general } 37108 \text { (def) }\end{array}$ & science 2523 \\
\hline general 37108 (def) & Laurén, Christer \\
\hline specific 37108 (def) & LSP $19252 f$ \\
\hline special form of 37108 (def) & and iconism 31219 \\
\hline specific $37101 \mathrm{f}$ & Lauridsen, Karen M. \\
\hline the term $3795 \mathrm{ff}$ & legal English \\
\hline powerless 36132 & can and may in $\mathbf{1 3} 146$ \\
\hline pro-drop 38192 & modals in 916 \\
\hline processing $631-61$ & law \\
\hline connectionists approaches to 6 & civil $3247 \mathrm{f}$ \\
\hline $31-61$ & commercial \\
\hline purpose of $\mathbf{1 6} 179$ & vs land 9119 \\
\hline reality-based $1784 f$ & common 3247 \\
\hline reform 22131 & construction rules 9117 \\
\hline scriptualization of 2494 & functions of 99 \\
\hline speaker-based 17 84f & the language of the 914 \\
\hline spoken and written 2494 & Latin 9124 \\
\hline the standard variant 17 114-116 & legal cultures 32173 \\
\hline tetratomy of $\mathbf{1 7} 85 \mathrm{f}$ & legislative information \\
\hline theory construction about $3304-307$ & packaging of $3256-64,72$ \\
\hline three-party two-language model & qualifications 3257 (def) \\
\hline $1044^{1}$ & legislative rules 32101 \\
\hline use & legislative systems \\
\hline and international business $\mathbf{3 8}$ & arbitration agreement $3227 f$ \\
\hline $105-114$ & arbitration procedures $3228 \mathrm{f}$ \\
\hline in professional settings 19 207- & and their languages $3247 \mathrm{f}$ \\
\hline 238 & see also courtroom discourse; \\
\hline and world 18 54-56 & legal acts; legal discourse; legal \\
\hline see also LSP & language; legal texts; legal writing \\
\hline angue 38108 & Law of Jante 27224 \\
\hline arsen, Fritz & layout \\
\hline English sentence analysis 12 186- & circus- 3452 \\
\hline 189,1711 & laypeople 18101 (def) \\
\hline ascar, Elizabeth & learning algorithm 6 38-41 \\
\hline interpreting 20222 & Leckie-Tarry, H. \\
\hline $\begin{array}{l}\text { asnik, H. } \\
\text { grammatical filter } 813\end{array}$ & $\begin{array}{l}\text { explicitness in communication } 28 \\
62\end{array}$ \\
\hline atin (=Hispanic/French etc.) & Lederer, Marianne \\
\hline vs Anglo-Germanic cultures 15 54ff & interpreting 1045 \\
\hline concepts of politeness and time & Leech, Geoffrey \\
\hline $1553-76$ & formators $572 \mathrm{f}$ \\
\hline atinisms & left dislocation 8 19f \\
\hline in legal English $9125 f$ & $\begin{array}{l}\text { legal acts } \\
\text { obligation } 679 f, 88,9102 \mathrm{f}\end{array}$ \\
\hline
\end{tabular}


performative statement 6 77-79 permission 6 81f, 88, $9103 \mathrm{f}$ prohibition 6 80f, 88, 9103 legal discourse 9 9-17, 22 113-133 across cultures 32 13-33 across languages 32 13-33 multicultural and multilingual contexts

generic integrity 32 7-11, 13-33 written $9116 \mathrm{f}$

the audience in $9116 \mathrm{f}$ see also courtroom discourse legal language 6 67-71, 68 (fig), 9 10-17, 12 (fig), 113-139, 13 145, 22 101-111

characteristics of $910 \mathrm{f}$ concepts 22 103-105

cooperation between lawyers and linguists 22132

criticism of writing style 9 134-136

development 31 219-226

English 9 11, 15

characteristics of 6 65-67, 9

118-129

use of argot 9124

capitalisation 9 119f

complexity elements $9120 \mathrm{f}$

extreme precision of expres-

sion 9124

use of formal words 9124

lexical markers 9 121-129

modern use of Latin 9 123-126

use of Old and Middle

English words $9122 \mathrm{f}$

use of terms of art 9123

visual markers 9119

whiz-deletion 9122

modal expressions 13 145-154

modal verbs

can and may 9 43-63

frequency of $\mathbf{1 3} 149$ (fig)

speech acts 6 65-88

regulative 9 83-109

functions of 9 114-117

German modal expressions 13 145-154

modal verbs

frequency of 13150 (fig)

obligations 13151

information loading and textual mapping $3226 \mathrm{f}$

vs the language of the law 668,9

$11 \mathrm{f}$

the legal translator 9 113-139

in the Malaysian Arbitration Act

32 77-97

legal texts

comprehensibility of 36 15-39

German

court decisions 36 15-39

linguistic methods in the description of $2299 \mathrm{f}$

legal writing 32 45-72

style 32 55f, 71

syntactic discontinuities 3281 (def)

textual organization 32 51-54

variation in $\mathbf{3 2} 49$ (fig) generic 32 45-72

legislative writing $\rightarrow$ legal writing

Lehmann, Christian

grammaticalization 38191

leísmo 38189

Leitner, G.

description of begin and start 13

104

Leki, I.

plagiarism 2814

Lelubre, Xavier

French and Arabic adjectives in

LSP texts 37154

lemma 451

stock 450 (def)

vs macrostructure $450 \mathrm{f}$

Lenat, Douglas B.

dilemma of AI 25120

Lenstrup, Rita

translation and simultaneous

interpreting $1475 f$

Leonard, Don

comma splice 3194 
students' grammatical errors 3194 teaching grammar $\mathbf{3 1}$ 96, 99

Lerat, Pierre

CTN 8 109, 1815

LSP dictionaries vs web resources 37153

letters

the body of 25 135-140

openings and closings in 25136

letters of application

textlinguistic and genological

approach 31 105-116

Letto-Vanamo, Pia

legal systems and legal languages 31220

Lévi-Strauss, C.

cultural systems $38107 \mathrm{f}$

Levinson, Stephen C.

contrasting messages 1114

dialogues $\mathbf{3 8} 45$

face want 2776

face-threatening acts 2776

in-group jargon $\mathbf{2 8} 78$

politeness $2775 \mathrm{f}$

strategies

vs facework 2776

Levy, Steven

computer metaphors 24146

Lewis, J.W.

conspicuous GB 17224

lexemes

status 29285

lexical density 3168 (def), $71 \mathrm{f}$

lexical vagueness $3470 \mathrm{f}$

lexicalism 16 191-195

Lexicalist Controversy 16 189-191

Lexicalist Hypothesis 10 12, 15

lexicography 8 101-110

active/passive theory 6102,8102 , 31171

problems about 8104

bilingual 8 101-104, 13 177f

classification of lexicographic

referenceworks 6 92-94, 106

functional 6 92-94, 106, 31 182ff phenomenological 6 92-94,

106, 31 178-185

Wiegand 31 178-185

communication model 31174 (fig)

courses in $\mathbf{1 8} 19$

van Dale's concept of L1-L2 dic-

tionaries 8106

editing and computerization $\mathbf{8}$

106-109

functional theory 31 171-194

lexicographic functions $\mathbf{6} 96, \mathbf{1 8}$

98-101, 31 171-194, 176 (def), 34

85,37110

cognitive $\rightarrow$ knowledge orien-

tated

communication-orientated 31

176, 3485,37110

communicative $\rightarrow$ communi-

cation-orientated

vs functionalities $\mathbf{3 4} 38 \mathrm{ff}$

knowledge-orientated 31 176f,

3485,37110

text dependent 3485

text independent 3485

text production and translation

18 107-112

lexicographical information costs

$3487 f$

comprehension-related $3487 \mathrm{f}$

search-related 3487

vs lexicology 37152

LSP

legal terminography 22 101-111

new methodology 22 101-111

and terminography 18 91-96,

119-121

and terminology 18 9f, 13-29

militant 37155

monolingual $8105 \mathrm{f}$

relevance theory applied to $696 \mathrm{f}$

structures

macro- 4 49-64, 50 (def), 51

(def), 52 (def)

back matter $451 \mathrm{f}$ 
complex 453 (def), 53f, 54

(fig), 64

defining macrostructure 4 49-52

front matter 4 51f

preface 461 (def)

fully developed 460

interrelationship 4 55-58

simple 452 (def), 52f, 53

(fig), 64

systematic vs alphabetical 6

$100 \mathrm{f}$

underdeveloped 459 (def)

vs terminography $\mathbf{1 8}$ 91-121, 37152

vs terminology 18 13-29

user situations

communication-orientated $\mathbf{3 1} 174 \mathrm{f}$

knowledge-orientated $\mathbf{3 1} 173 \mathrm{f}$

see also dictionaries; spelling

checkers

lexicology

vs lexicography 37152

lexicon (mental) 17 97-101

axiological parameter

according to Faber and Mairal

30 182-186

axiological pattern

implementation 30 186-195

English

axiological evaluation 30 179-196

functional grammar 30179

Lexin (Svensk-Finskt Lexikon)

design 34 47-52

Lexinome 18113

lexis

social groups 28 59-79

LFG 16194

LGP $\rightarrow$ language

Li, L.

Chinese academic writing $2524 \mathrm{f}$

Lind, Allan

powerless language 36132

Lindgren, Astrid

translation 1238

Lindstrøm, Anna

requests $3871,74,77$ linear threshold units 636

linearization rules $\mathbf{1 0} 24$

Linell, Per

interpreting and communication

22162

miscommunication 1582 (def)

monologism vs dialogism 14114

recontextualization $\mathbf{2 8}$ 90, 3265

lingua franca

negotiating in a 15 9-27

linguistic change 38 190-192

in Spanish 38 192-194

linguistic competence

and cross-cultural negotiations $\mathbf{1 5}$

9-27

levels of 15 19-21

linguistic range $\mathbf{1 3} 205$

linguistic relativity, theory of $\mathbf{1 2} 10$ see also Sapir-Whorf hypothesis

linguistics $\mathbf{1 6} 200$

contrastive

and translation studies 26 69-71

corpus 139

concepts 29287

data 29287

methods 29287

the functional paradigm 6 182, 38

196

linguistic creativity 6181

linguistic functions 17 83-86

linguistic normalization 10 53-64

linguistic supertypes 17 83-86, 85

(fig)

the problem of method $2181-183$

self-deception 3 287-289

the purpose of linguistic description 16200

research 2 179-185, 3 287-308

self-deception 3 287-289

socio- 13 135-142

accent as social marker 17216 -

218

metropolitan sub-variety $\mathbf{1 7} 227 \mathrm{f}$

regional prestige 17 224-226 
Stockholm Swedish spoken by youngsters $\mathbf{9} 216$

urban 9 211-216

the structural approach critique against $\mathbf{3 8} \mathbf{1 0 8 \mathrm { f }}$

text 16 9-13

Centre for Text Linguistics (in Denmark) 16 9f

theories 2 180f

causalistic theory $\mathbf{3} 306$

deductive-nomological theory 3

$306 \mathrm{f}$

functionalistic theory $\mathbf{3} 306$

theory of linguistic relativity $\mathbf{1 2}$ 10

theory construction about language 3 304-307

see also descriptivism; language; prescriptivism

links 17 65f

extra-hypertextual 1765

inter-hypertextual 1765

intra-hypertextual 1765

LIP $\rightarrow$ London Interpreting Project

Lipka, L.

semantics of begin and start $13103 \mathrm{f}$

listing $25102 \mathrm{f}$

Livesey, Sharon

business environmental discourse 2759

LLC $\rightarrow$ London-Lund Corpus

loan words

in French LGP dictionaries 37155

LOB-Corpus 9 44, 1348

begin and start in 13 105-120

locale 36 199-201

the concept 36200

the term 36209

localisation 12 42, 44-48, 32 190-193, 191 (fig), 36 197-216, 197 (def), 209 (def)

Eastman Kodak $36202 f$

and process management 36 201203 tourist brochures 32193 (fig)

vs translation 36210 (fig)

locality 36 199-201

LoCastro, Virginia

plagiarism 287

Lodge, David

Therapy $2755 \mathrm{f}$

Lörscher, Wolfgang

translation $\mathbf{1 0} 44$

$\log$ files

of reverbalization processes as a method in comprehensibility research 37 67-89

as tool for improving internet dictionaries 34 117-140

logic 5 70f, 81, 93

and connectors 5 84-92

logical forms 28 126f

logos 3749

loísmo 38189

LOKE 25116

Lomheim, Sylfest

dialogue and trilogue 1236

London Interpreting Project 12135

London-Lund Corpus 13 47-52, 157

Longman Dictionary of Contempo-

rary English 3 125ff

Longman Dictionary of English

Language and Culture

biotechnological terms in mono-

lingual dictionaries 18 103-107

Longman/Lancaster Corpus 2330

Lord Kelvin

performance measurements 2710

Lorenzo, M.P.

translation 23207

Louhiala-Salminen, L.

letters of application 31107

Lowrey, G.

business letters 1567

LSA $\rightarrow$ Lag Sequential Analysis

LSP 8 127-131, 131 (def), 23 25-

40, 2566 (def), 69f, 37 9-20, 37-

41, 151-159

-communication 25 66-68 
two bibliographies about 2 133-135

characteristics 2567

communication 37 9-20

doctor-patient communication 23

250-254

9th Europen Symposium on 19

251-261

horizontal/vertical distinction 6

69f, 70 (fig)

and the layman 2568

legal language 6 67-71, 68 (fig), 9

10-17, 12 (fig), 113-139, 13 145,

22 101-111

characteristics of $910 \mathrm{f}$

concepts 22 103-105

cooperation between lawyers

and linguists 22132

criticism of writing style 9 134136

development 31 219-226

English 9 11, 15

characteristics of 6 65-67, 9

118-129

use of $\operatorname{argot} 9124$

capitalisation 9 119f

complexity elements $9120 \mathrm{f}$

extreme precision of expres-

sion 9124

use of formal words 9124

lexical markers 9 121-129

modern use of Latin 9 123-126

use of Old and Middle

English words $9122 \mathrm{f}$

use of terms of art 9123

visual markers 9119

whiz-deletion 9122

modal expressions 13 145-154

modal verbs

can and may 9 43-63

frequency of 13149 (fig)

speech acts 6 65-88

regulative 9 83-109

functions of 9 114-117

German

modal expressions 13 145-154 modal verbs

frequency of $\mathbf{1 3} 150$ (fig)

obligations 13151

information loading and textual

mapping $3226 f$

vs the language of the law 6 68,

9 11f

the legal translator 9 113-139

in the Malaysian Arbitration

Act 32 77-97

legal terminography 22 101-111

new methodology 22 101-111

vs LGP 6 105, 2566

medical language

classical terms 25 11-13

hybrids 25 11-13

Danish 25 19f

morphological structure of

terms 25 13-19

the nationalisation of terms $\mathbf{2 5}$

$11-20$

transformation of classical

terms into Danish 2513

metaphors in 24 9-12

and neologisms 8 109f

purpose of $\mathbf{3 7} 115$

technical language $2370 \mathrm{f}$

translation of $\mathbf{6}$ 106, 10 115-123,

23 65-74

see also administrative language; convergent subjects; discourse; divergent subjects; negotiation language; rural subjects; urban subjects

LSP lexicography

legal terminography 22 101-111

new methodology 22 101-111

and terminography 18 91-96,

119-121

and terminology 18 9f, 13-29

see also dictionaries; lexicography

LSP research 19 251-261

LSP texts

economics texts

English 23 25-40 
external variables $2336 \mathrm{f}$ internal variables 23 28-36

focal terminology 23 32-34

lexical aspects 23 29-32

pronouns in 23 34-36

German

legal texts

comprehensibility of 36 15-39

German

court decisions 36 15-39

linguistic methods in the

description of 22 99f

level of specialization 37154

lexical inventory 37116

medical texts 25 63-73

translation 25 49-59

metaphors

translation of 24 57-79

optimizing 37 115-126

readability of

English vs German 37 116ff

reformulation phenomena 37154

technical texts 8 129f, 21227

(def), 23 71f

expressivity in $\mathbf{2 1} 225-231$

prototypical 2366

the technical writer 2367

terminology in $\mathbf{2 3} 73$

translation 23 65-74

LSP translation 6 106, 10 115-123,

12 19f, 23 65-74, 37 115-126

equivalence $1214 \mathrm{f}$

legal language courses for translators 22 131f

the legal translator 9 113-139

of metaphors 24 57-79

technical translation 21 225-231,

23 65-74

vs literary translation $\mathbf{2 3} 68 \mathrm{f}$

the state of 23 67-69

and terminology $\mathbf{1 8} 18 \mathrm{f}$

Luckmann, $\mathrm{T}$.

genre 2394

Luppescu, $\mathrm{S}$.

corrective feedback 1593
Lyons, John

medium-transferability $\mathbf{1 3} 46$

social gender $1257 \mathrm{f}$

structuralism 21228

\section{M}

McArthur, Tom

levels of linguistic competence $\mathbf{1 5}$

20

Macauley, R.

Received Pronunciation 17224

McCarthy, John

artificial intelligence 25119

McClelland, J.L.

past tense simulation 6 43-48

McDonald, P.F.

special language 2566 (def)

vs general language $\mathbf{2 5} 66$

machine translation

knowledge representation in $\mathbf{1 0}$

118, 38 223f

knowledge-based 38224

Macmillan English Dictionary for

Advanced Learners 34 12ff

Macnamara, $\mathrm{J}$.

code-switching 15 45f

macrostructures (lexicographic) 4

49-64, 50 (def), 51 (def), 52 (def)

back matter $451 \mathrm{f}$

complex 453 (def), 53f, 54 (fig), 64

defining 4 49-52

front matter 4 51f

preface 461 (def)

fully developed 460

interrelationship 4 55-58

simple 452 (def), 52f, 53 (fig), 64

systematic vs alphabetical $6100 \mathrm{f}$

underdeveloped 459 (def)

Madsen, B. Nistrup

defining concept 8130

dictionary database $\mathbf{8} 109$

Madsen, Karin L.

legal interpreting in Denmark 20

217 
Maegaard, Bente

knowledge representation in MT

10118

Magalhães, João

a multilingual medical dictionary

$37157 f$

Maidahl, Lisbeth

use of LSP dictionaries 10122

Maidment, J.A.

boundaries between Cockney and RP 38181

Maier, Elisabeth

abstracting 25127

Maier, P.

business letters $\mathbf{1 5} 68$

Mairal Usón, Ricardo

axiological evaluation 30195

lexical structure and cognition 30 180

Malaysian

academic writing

criticism in 2525

Malinowski, Bronislaw

critique against structuralist lin-

guistics $\mathbf{3 8} 108$

Malmkjær, Kirsten

translation 13169

management

conflict

in price negotiations 15 111-139

control 27 9-28

the control situation 27 15-17

and culture 15 55-60, 71

division 27 9-28

ethos appeal 2724

performance measurements $279 \mathrm{f}$ and social erosion 27 10-14

success 15 53-76

training 15 70-75

Mandelblit, N.

blending 24139

Maniez, François

term measurement $37151 \mathrm{ff}$

terminologically relevant relational adjectives $\mathbf{3 7} 153 \mathrm{f}$
Manipulation School 12 15f

in a contemporary perspective $\mathbf{1 2}$

$70-72$

in a historical perspective $1269 \mathrm{f}$

and interpretation research 12 82-86

manoeuvring

relational 1540

tactical 1540

markedness 38 190-192, 190 (def)

in Spanish 38 192-194

marketing

bank $2731 \mathrm{ff}$

customer partnering $2731 \mathrm{ff}$

green

the paradoxes of 27 59-61

relationship building $2731 \mathrm{ff}$

relationship marketing

and marketing communication

$2733 \mathrm{f}$

vs traditional marketing 2732

Martin, J.R.

genre 16 40, 44f, 48, 53f

communicative purpose $2318 \mathrm{f}$

concept of $\mathbf{2 3} 22$

theory 2314

intercultural communication 15 74f

Martinéz, Inmaculada Penadés

Spanish semantics and syntax 6186

Martínez, Jesús M.

translating for EU 34 293-297

Martinsen, B.

media and court interpreting 22166

Martinsen, N.

court interpreting 20227

Mason, Ian

equivalence 1214

literature vs non-literature 2372

text and translation 2612

text types $\mathbf{2 3} 74$

translation 21 227, 2617

Masuko, Mayumi

plagiarism 287

Mattila, Heikki E.S.

legal language $31219 \mathrm{ff}$

legal linguistics 31 222-225 
Mauffette-Leenders, L.A. interpreting $20222 \mathrm{f}$

maxims of conversation $\rightarrow$ conversation

Mazeland, Harrie interviews 32 119f, 132, 134 meaning 3 288-308, 5 69ff, 14

113f, 20 165-179, 29 285-289

vs form 1240

identification

in the translation process 619 (fig)

importance of phraseology 29286

language

illusions about 3 289-292

language-internal 3293

model of $\mathbf{3 7} 15 \mathrm{fig}$

and person-external reality 3301 304

personal-internal 3 292-307

self-deception 3 287-289

theory construction about 3 304-

307

see also sense

MED $\rightarrow$ Macmillan English Dicti-

onary for Advanced Learners

MediaMOO 24 85f

medical language

classical terms 25 11-13

hybrids 25 11-13

Danish 25 19f

morphological structure of terms

25 13-19

the nationalisation of terms 25

11-20

transformation of classical terms into Danish 2513

medical texts 25 63-73

translation 25 49-59

Meeuwis, Michael spoken Spanish 13331

Mejri, Salah

idioms 3549

Mellon, Sir James

Denmark is homogeneous 27224 memes 38222

memetics 38222

memetics of knowledge $\mathbf{3 8} 222 \mathrm{f}$

Menac, Antica

European idioms 3547

mental space theory $\mathbf{3 4} 202,206$

mental spaces 24 139, 34 202, 38229

meta-labels $\mathbf{1 1}$ 57, 59

metadiscourse

classification of 31113 (fig)

metadiscursivity 31 112-116

metaphor theory 20 167f, 35 185-188

cognitive 20 168, 35 185f

comparison 20167

conceptual $35183 \mathrm{f}$

interaction 20167

interactive 20167

substitution 20167

metaphorical mapping 35 185f, 189

metaphors 5 49, 56, 18 80-83, 20

165-179, 2460 (def), 81f, 113 (def)

computer 24146

and computers 19255

conceptual 24 138-144

conventional 20177

dead 20177

desktop 24145

conceptual mapping of $\mathbf{2 4} 145$

interface as $\mathbf{2 4} 145$

in dictionaries 20169

the essence of 24 111-114

explicitly vs implicitly signalled

2449

in fiction 20168

in German and Danish articles about business cycles 24 57-79,

70 (fig)

identifying 35 194-202

and the ideology of information

technology 24 131-158

interpretation $\mathbf{2 4} 72$

in LSP 24 9-12

a metaphor called Mozart 24 109-

130 
metaphoric processing vs metaphor processing 2449 metaphorical idioms 35187 metaphorical utterances 552 vs metonymy 20 177, 35187 and music $24124 \mathrm{f}$ in non-fiction 20168 the notion of context-dependent criteria 24 60-66 original 20178 pictorial

in computer advertisements $\mathbf{2 4}$ 31-55

primary 35186

the problem of categorising $2468 \mathrm{f}$ realisation of 24 119-121

reflections on the use of 24 15-29 revitalized 20178

source domains used in $2452 \mathrm{f}$ in specialised language 24 9-12 translating 35 183-205

in LSP texts 24 57-79

types of 24 109-111, 35184

the use of

in computing 24 15-29

see also metonymy; tenor; vehicle metonymic mapping 35187 metonymy 20 177, 35187 vs metaphor 20 177, 35187

Mexican

business letters 37 53f, 60f

negotiation behaviour 15 9-27

vs Norwegian negotiators $\mathbf{1 5} 18$

Michael, S.

community interpreting 20228

Mikelonis, Victoria M.

special topics $3754 \mathrm{f}$

Mikkelsen, Hans Kristian self-knowledge of dictionaries $\mathbf{1 0}$ $120 \mathrm{f}$

Mikkelson, Holly

community interpreting 20215

court interpreters 20226

Mikroøkonomisk Glossar

review 9 207-210
Millard, Derrick

teaching grammar $3198 \mathrm{f}$

Miller, Carolyn R. genre 16 41f, 48, 23 94, 3716 (def) special topics 3751

mind (human)

the structure of 17 80-86

Mingorance, L.M.

functional grammar lexicon $\mathbf{3 0} 179$

functional lexematic model $\mathbf{3 0} 180$

Minimalist Programme $16198 \mathrm{f}$

Mintz, $\mathrm{H}$.

court interpreters 20226

miscommunication 12 176f, 1582

(def), 3759

affective 1582

cross-cultural 1566

incipient 15 81-108

referential 1582

relational 1582

sociocultural 15 97-106

types of $\mathbf{1 5} 86-92$

discourse structural 15 88-97, 106 lexical 15 86-88, 92-97, 106

Mishler, Elliot G.

interviews 27 179, 32121

Miyake, Yutaka

simultaneous interpreting 14137

Mizuno, Akira

intercultural communication,

negotiation and interpreting 22165

interpreting

media and court 22166

research 14139

in Japan $1412 \mathrm{f}$

modal logic 946

modal operators 7 168, 947

modality 945 (def), 46 (def), 31

229-239

cross-

interactional 12147

deontic 7 169ff, 9 46f, 49

dynamic 947

English 13175

epistemic 7 167ff, 9 46f, 49, 58 
196

German 13175

and the indefinite article 31 234-236

modal concepts 13 74-76

and their exponents 1371 (fig)

modal expressions 17279 (def)

in German and English legal

texts 13 145-154

a frequency analysis 13 145-154

modal source 946 (def)

vs mood 7170

necessity $17 \quad 118-120$

non-epistemic 7 167ff, 958

non-possibility 955

epistemic 9 53-55

root 954

obligation 17 118-120

permission 17 118-120, $32102 \mathrm{f}$

possibility $17118-120$

deontic 7 172, 9 53-57, 62

constative 9 56-62

performative $961 \mathrm{f}$

dynamic 7 172, 9 53-55, 62

epistemic 7 172, $953 \mathrm{ff}$

non-epistemic $953 \mathrm{ff}$

non-performative root 954

$\operatorname{root} 949-62$

modification 31 65-79

modificators 15 46f

modifiers 31 65-79, 65 (def)

function analysis 31 73-75

modularity

principle of $3460 \mathrm{f}$

Møller, Bernt

administrative language 3135

translation of LSP texts 10122

Møller, Erik

sociolinguistics 9215

Mørk, Henning

LSP 10117

Moeschler, Jacques

text linguistics 16 11f

Moitra, S.D.

English academic writing 2524

Mokienko, Valerij M. internationalisms, Europeanisms and universality $\mathbf{3 5} 48$

Mondahl, Margrethe

information processing strategies

31 10f

translation processes by means of

introspection 1476

monitoring $\mathbf{3 6} 10$

monologism $14113 \mathrm{f}$

Montolío, Estrella

conditional sentences $\mathbf{1 3} 331$

MOO 24 84-93

Media- $2485 \mathrm{f}$

$\operatorname{mood} 1794 \mathrm{f}$

vs modality 7170

tense and mood in English vs

Danish 7 163-174

Moravcsik, M.

English academic writing 2524

morphology

basic units and word elements 25

$14 \mathrm{f}$

structure of medical terms 25 13-19

morphs 2514 (def)

Moser-Mercer, B.

skill components in simultaneous

interpreting 22164

two groups of interpreters 1276

natural science community $\mathbf{1 2} 78$

Motta-Roth, D.

English academic writing 2524

Mourier, Lise

Translation Process Diagram 32 159 (fig)

move-structure 19 211, 22 114,

116-119, 27 65-69, 74f, 31 39-41

at macro-level 22 116-118

moves 19 215, $236 \mathrm{f}$

ambiguous 11 35f, 42-44

classification of $\mathbf{1 1} 31$

disguised $\mathbf{1 1}$ 31, 35f

linked $\mathbf{1 1}$ 31, 35f

at macro-level 22116

at micro-level 22116

pull 1128 (def), 33f, 39-42 
acknowledging moves 11 34, 41 Hispanic vs Swedish 11 27-46 we-strengthening 1134 yielding 11 33f, 41 push 1128 (def), 31-33, 36-39 argumentative 11 31f, 36-38 assertive 11 32, 38f basic initiatives $\mathbf{1 1}$ 31f, 36 Hispanic vs Swedish 11 27-46 MS theory $\rightarrow$ mental space theory $\mathrm{MT} \rightarrow$ machine translation MUD $\rightarrow$ Multi User Dungeon Mudersbach, Klaus culture $\mathbf{3 8} 231$ (def)

Mühlhäusler, $P$. greenspeak 2758 (def)

Mugdan, J.

dictionary grammar 38120 (def), $120 \mathrm{ff}$

Mulder, Gijs

business letters 1567

pragma-linguistic approaches $\mathbf{1 3}$ $331 \mathrm{f}$

Muller, Charles

Orthonet 37155

Multi User Dungeon 24 84-93

Multi User Dungeon Object Oriented $\rightarrow$ MOO

multi-layered perceptron 634 (fig) multinomials 3259 (def), 80 (def)

Munck, Lena

LSP 8129

municipalities 3813

Muñoz, C.A.

translating medical texts 259

Murugesan, P.

English academic writing 2524 music

and metaphors $24124 \mathrm{f}$

MYCIN 25116

Myers, G.

parameters for advertising 23100

Myking, Johan

LSP research 19 251-261
$\mathbf{N}$

NAFTA

culture in the EU and NAFTA

areas 15 55-60

NAFTA market

size of $\mathbf{1 5} 59 \mathrm{f}$

Nagata, Sae

simultaneous interpreting $\mathbf{1 4} 133 \mathrm{f}$

narratives 9215

Natlandsmyr, J.H.

cross-cultural negotiations $1523 \mathrm{f}$

navigation tools $1765 \mathrm{f}$

near-copying 2840

necessity 17 118-120

Nedergaard Thomsen, O.

subject predicative $38198 \mathrm{f}$

negating elements

forms and patterns of realisation of $989 \mathrm{f}$

negotiation culture 1557

English 1557

German 1557

Oriental 1557

Romance 1557

Slavic 1557

negotiation language

Danish vs English 11 11-23

foreign language 11 11-23

in management training 11 49-59

negotiations 3196 (def), 1161 (def), 159 (def), $107 f$

aspiration level $3202 \mathrm{f}$

behaviour 3198

camouflaging style 1117

characteristics of $\mathbf{1 1} 62 \mathrm{ff}$

commercial 3197 (def)

communication

difficulties 3 210-215

dimensions 1165

skills 3 209-215

conflict management

in price negotiations 15 111-139

contents 3198

cooperation 


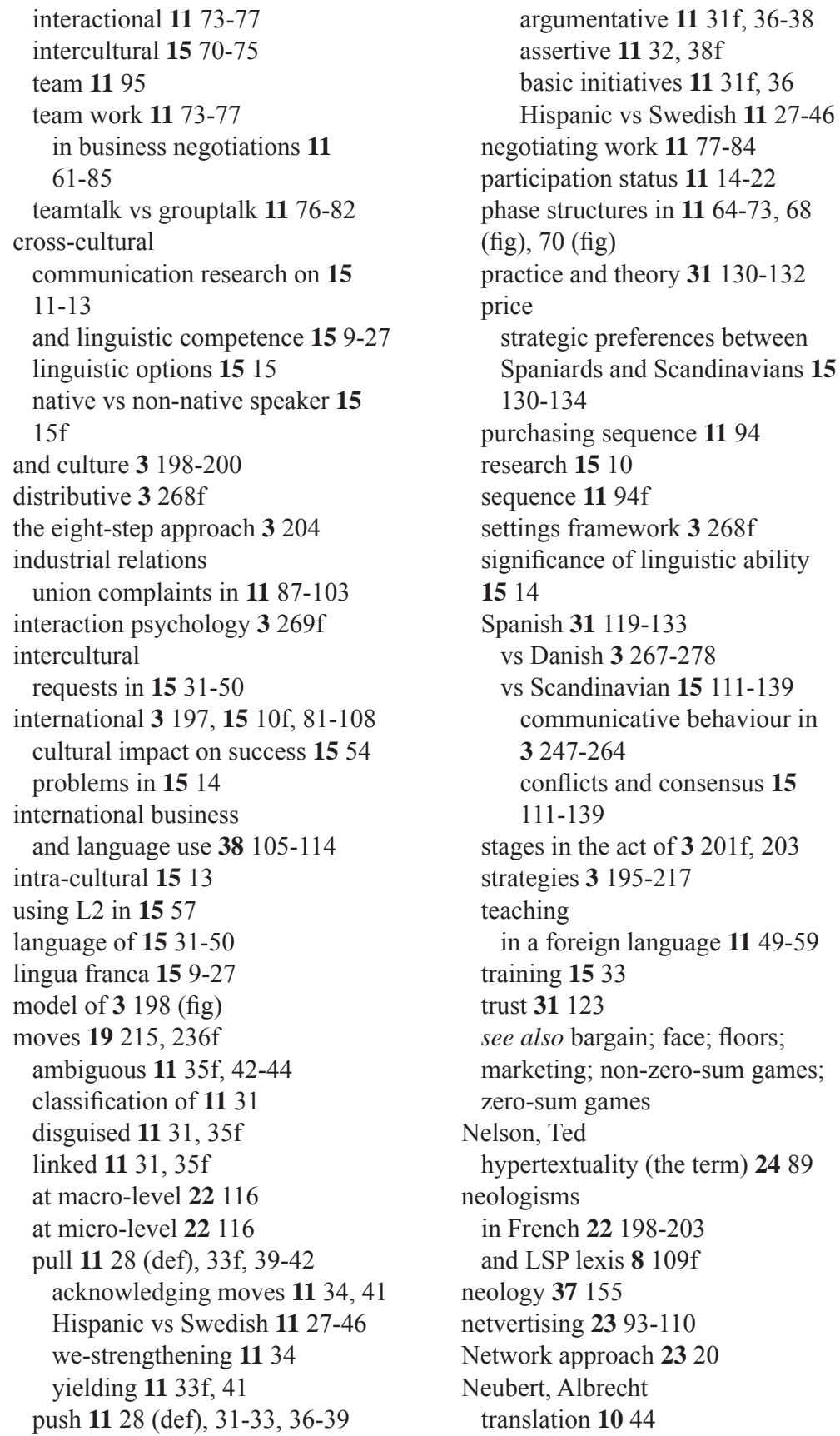

argumentative 11 31f, 36-38

assertive 11 32, $38 \mathrm{f}$

basic initiatives 11 31f, 36

Hispanic vs Swedish 11 27-46 negotiating work 11 77-84

participation status 11 14-22 phase structures in $\mathbf{1 1}$ 64-73, 68 (fig), 70 (fig) practice and theory 31 130-132 price

strategic preferences between Spaniards and Scandinavians 15 130-134

purchasing sequence 1194 research 1510

sequence $1194 \mathrm{f}$

settings framework $3268 \mathrm{f}$

significance of linguistic ability 1514

Spanish 31 119-133

vs Danish 3 267-278

vs Scandinavian 15 111-139

communicative behaviour in 3 247-264

conflicts and consensus $\mathbf{1 5}$$$
\text { 111-139 }
$$

stages in the act of $3201 \mathrm{f}, 203$

strategies 3 195-217

teaching

in a foreign language 11 49-59

training 1533

trust $\mathbf{3 1} 123$

see also bargain; face; floors; marketing; non-zero-sum games; zero-sum games

Nelson, Ted

hypertextuality (the term) 2489 neologisms

in French 22 198-203

and LSP lexis 8 109f

neology 37155

netvertising 23 93-110

Network approach 2320

Neubert, Albrecht translation $\mathbf{1 0} 44$ 
studies 31205

Neumann, Ingrid

language of negotiation 1110

Neumann, Stella

legal communication 36 10f

neural networks $631 \mathrm{f}$

artificial 6 32-42

representation and learning in $\mathbf{6}$ 34-42

Neuromancer $2419 \mathrm{f}$

Nevalainen, Terttu

paradigms 13158

The Helsinki Corpus of Early

English Correspondence 13135

New Corpus of Spoken English $773 \mathrm{f}$

New Oxford English Dictionary 12

$214 \mathrm{f}$

new-rheme 21201

Newmark, Peter

metaphors 20177

types of $\mathbf{3 5} 184$

technical terminology 2373

technical writing 2372

translation 12 68, $2550 \mathrm{f}$

denying 2550

of technical texts 21227

Newmeyer, F.J.

grammaticalization $\mathbf{3 8} 191$

Nicholson, N.S.

community interpreting $\mathbf{2 0} 215$

competent interpreters 1224

court interpreting 20227

interpretation research and training 22163

Nickl, Markus

legal communication 36 10f

Nida, Eugene A.

concept of equivalence 1211

meaning vs form 1240

presuppositions of communication

1232

translation $16202 \mathrm{f}$

Nielsen, Anne E.

business rhetorics 278
Nielsen, J.E.

neologisms 12 212f

translation/lexicography 12 210ff

Nielsen, Joel N.

instruction manuals 10117

translation 1220

Nielsen, S. Juul

nominalizations in Danish 8131

Niemi, Jussi

prosody 3324

Nietzsche, Friedrich

reality $\mathbf{2 4} 11$

Nishio, Michiko

simultaneous interpreting $\mathbf{1 4} 132 \mathrm{f}$

Nissen, Uwe K.

translation 1219

Nobes, Christopher

translation 32147

nodes

constituent-nodes $\mathbf{1 0} 9$

Nørreklit, Hanne

business rhetorics 278

nominal categories 17 95-97

internal structure 17 95-97

nominal characters 3280 (def)

nominal gender $\mathbf{1 7} 95 \mathrm{f}$

nominalization 10 12-15

non-argument chain 816

non-assertiveness $\mathbf{1 2} 151 \mathrm{ff}$

non-diegetic insert 34209 (def),

216 (fig)

non-perfect

vs perfect

English and Danish 7166

non-possibility 955

epistemic 9 53-55

$\operatorname{root} 954$

non-renditions 12175

non-zero-sum games $\mathbf{3} 268$

Nord, Christiane

skopos theory 21226

translation 12 23, 16 206f, 214,

216f, 25 51f

Nordhjem, Bent

NPVs in English 12 210f 
Nordic languages 12 215-217

Norlyk, Birgitte

translation and communication 23 10

norms 1272 (def)

concept of 12 72-74

operational 1273

preliminary 1273

Norrby, Catrin

pause fillers 36140

Norwegian

Bokmål 1054

communicative behaviour

vs Spanish 3 247-264

cultural differences between

Spaniards and Scandinavians 15

115-117

linguistic database for $\mathbf{1 0}$ 54-56

vs Mexican negotiators 1518

as native and foreign language $\mathbf{1 5}$

$35 f$

negotiation behaviour 15 9-27

negotiations

vs Spanish

conflicts and consensus 15

111-139

strategic preferences negotia-

ting price 15 130-134

Nynorsk 1054

Norwegian Language Council 10

55-57

nouns 12190 (def)

active $\mathbf{3 1} 36$

discontinuous groups 17 50-54

nominal gender $\mathbf{1 7} 95 \mathrm{f}$

phrasal 3136

Novak, T.P.

banner ad 23101 (def)

NP

the internal structure 10 16-19

NP-movement 89

NP-trace 8 11, 18

received NPs

idiomatic, syntactic and collo-

\author{
cational characteristics of $\mathbf{1 3}$ \\ $19-40$
}

NPV

English 12 210f

nucleus 13126 (def)

position 13 126-130

in the Spoken English Corpus 13

123-133

number (grammatical category) 1796

Nurmi, Arja

The Helsinki Corpus of Early

English Correspondence 13135

Nuwe Woordeboek sonder grense

vs Basiswoordeboek van

Afrikaans 38 217-220

review 38 217-220

Nynorsk $\rightarrow$ Norwegian

\section{$\mathbf{O}$}

O. Nagy, Gábor

Hungarian and Finnish phrase-

ological units $\mathbf{3 5} 49$

O'Barr, William

courtroom language 36 132ff

powerless language 36132

object 1015 (def), 1996

alternation 38 199f

indirect

in predicative constructions $\mathbf{3 8}$

196-199

Spanish 38 187-202

Obler, L.K.

code-switching 1546

obligation (legal language) 32103 -

105

the expression of 13 147-151

obligation (modality) 17 118-120

Occitan

clitics in 19101

Öberg, Britt-Marie

negotiations 1110

Oehlenschläger, A.

translation of J.W. von Goethe $\mathbf{1 2}$ $47 \mathrm{f}$ 
Østergaard, Frede

progressive aspect in Danish 1476

Offentlig Information Online 3818

Okamura, A.

pragmatics 21 69f

Olevard, Helena

gender-based language differences 36153

pauses 36139

women's and men's speech 36136

Olshtain, E.

complaints 2172

Online Writing Laboratory 1766

ontological categories 1857

ontology 38225 (def)

for translation purposes $\mathbf{3 8} 225 \mathrm{f}$

orality

oral vs written performances $\mathbf{1 4} 74$

principle of 32240

organisations 38 13f

Organon Model (Bühler's) 1784

(fig)

Oriental

negotiation culture 1557

Orlikowski, W.J.

genres 2395

Orthonet 37155

Ortony, Andrew

metaphors $249 f$

Osaka, Mariko

interpreting and the working

memory 14 134f

Osborn's Concise Law Dictionary

macrostructure 4 54ff

OWL $\rightarrow$ Online Writing Laboratory

Oxford accent $17213 \mathrm{ff}$

Oxford Advanced Learner's Dictio-

nary of Current English 3 125ff

Oxford Dictionary of Current Idio-

matic English 7130

Oxford English Dictionary 29285

\section{$\mathbf{P}$}

Pace, Wayne R.

policies $3814 \mathrm{f}$ (def)

Padilla, P.

interpreters working memory

span 2266

Page, Ben

public services 3866

Page, N.R.

Latin (=Hispanic/French etc.) vs

English culture 1558

Palander-Collin, $\mathrm{M}$.

The Helsinki Corpus of Early

English Correspondence 13135

Palmer, J.S.

ecospeak 2758 (def)

Palmer, Juan C.

advertising on the Internet 2310

Palmgren, Sten

legal Swedish and legal Finnish 31222

Palomares, Nicholas

gender-based language differences 36153

in institutional discourse 36146

Paltridge, B.

genre 239

par foc $\rightarrow$ focus

paraculture 1440

paradigms 1276 (def), 13158 (def)

the compromiser paradigm 13

159-165

typology $13158 \mathrm{f}$

Paradis, M.

code-switching 1546

parallel distributed processing

$\rightarrow$ PDP

Paredes, E.M.G. de

verbs 6186

Paris School 1276

Parker, Bob

translation 32147

parole 38108

participation 37 15f, 20 
passive 19 76f, 87f, 22121

anti- 19 77-79

backgrounding $1984 \mathrm{f}$

foregrounding $1984 \mathrm{f}$

French 19 79f

reflexive verbs 19 75-90

and reflexive $1980 \mathrm{f}$

in French reflexive verbs 19 75-90

medio- 19 81, $88 \mathrm{f}$

patchwriting 2840

pathos 3749

patient package insert 25 64, 68-73

Danish PPIs 25 69

and product summary $2565 f$

patterning

contrastive 12149 (def), 155

cross-modal 12 149, 151

redundant 12149 (def)

pause fillers 36139 (def), 139f

pauses 36139 (def)

PDP 6 33f, 47

PE77 781

Pedersen, E.M.

defining synonymy 12214

Pedersen, Inge Lise

urban sociolinguistics 9 211-216

Pedersen, Jette

contrastive dictionary grammars

38130

Pedersen, Karsten

service information pamphlets $\mathbf{3 1} 10$

Pedersen, P.A.

public services 38 66f

Peirce, C.S.

connectors $\mathbf{5} 82$

icons 586

Penney, Christine

community interpreting 20221

Pennycook, A.

plagiarism 2814

Perceptual Symbol Systems $34202 f$

Perez, Bertha

communication expectations 3762 perfect

vs non-perfect
English and Danish 7166

performance

linguistic 14 57f, 61f

Pergnier, Maurice

translation $\mathbf{1 0} 44$

Perkins, D.N.

information bank 1768

Permic languages 3557

permission 17 118-120, $32102 \mathrm{f}$

persuasion 31 65-79, 3749

Petersen, $\mathrm{T}$.

active vs phrasal nouns $\mathbf{3 1} 36$

Pharos Woordeboeke Dictionaries

5-in-1 3417

pheme 12202

phonetics

socio- $9214 \mathrm{f}$

phraseological false friends 35 66-68

phraseologisms

inter- 3548

phrases 7131

with an infinitive verb form

in Romance languages 19 93-111

phrase structure rules $1011 \mathrm{ff}$

physics

use of nuclear physics 16178

Picone, Michael D.

anglicisms and neologisms in

French 22 198-203

picture verbalization 30 199-211

Piehl, Aino

influence of EC legislation on

Finnish legal language 31221

piestis $\mathbf{3 7} 49$

Pilbeam, Adrian

negotiation training 1533

Pilegaard, Morten

LSP dictionaries 3130

Pinker, S.

language acquisition 6 46-48

Place, U.T.

complaints 2172

plagiarism 28 7, 13 (def), 39-56, 53

(fig) 
Japanese university students $\mathbf{2 8}$

16-31

Learners of English 28 11-31

reasons for $\mathbf{2 8} 12$

see also near-copying; patchwrit-

ing

Plato

beauty 24119

Great Chain of Being $\mathbf{3 0} 184$

Plested, M.C.

translating medical texts 259

Pöchhacker, Franz

D. Seleskovitch 1277

interpreting

community $\mathbf{2 0} 215,223$

conference 2169

research policy 22163

simultaneous 1411

vs translation 1473

orality $\mathbf{3 1} 204$

Polanyi, Michael

knowledge 25117

PoliceSpeak 25118

policies 38 14f (def)

Polish

Polish academic writing 2525

politeness 15 67f, 27 75f

behaviour

cross-cultural 15 53-76

concepts of

Anglo-Germanic and Latin

(=Hispanic/French etc.) 15 53-76

strategies $\mathbf{1 5} 61$

vs facework 2776

negative receiver-oriented 2779

positive receiver-oriented $\mathbf{2 7} 79$

verbal 1562

polyphony

structure of 5 73-77

theory of 5 73-77

polysemy 1322

Popper, Karl R.

anti-essentialist exhortation $\mathbf{3 1} 198$

challenging inductivism $2181 \mathrm{ff}$

the value of clarity $\mathbf{3 1} 199$
Porsch, Peter

Europeanisms 3548

PORTAL 31 47-64

Portuguese

clitics in 19 98-103, 110, 20 126-

128, 130-136

European vs Brazilian 20133

Posner, Roland

climbing 19 104, $108 \mathrm{f}$

conjunctivity 576

connectors $577 \mathrm{ff}$

formators 572

verbal functions 1997

possibility $17118-120$

deontic 7 172, 9 53-57, 62

constative 9 56-62

performative $961 \mathrm{f}$

dynamic 7 172, 9 53-55, 62

epistemic 7 172, 9 53ff

non-epistemic $953 \mathrm{ff}$

non-performative root 954

root 9 49-62

see also non-possibility

possible worlds, the concept of $945 \mathrm{f}$

Postal, Paul

T-rule 16191

Posteguillo, Santiago

advertising on the Internet 2310

potential function 12143 (fig)

Poulsen, Sven-Olaf

LSP dictionaries 3136

POV-shot 34209 (def)

power distance 1558

PP

complex 3280 (def)

internal structure $1021 \mathrm{f}$

PPI $\rightarrow$ patient package insert

practisearchers 1282

prepositions

discontinuous groups 17 54-56

pragmalinguistic competence $\mathbf{2 1} 67$

pragmatic theory $535 \mathrm{f}$

pragmatics 5 35-37, 6 182, 3476

business 21 69-71 
Chinese and French at the utterance level 13 351-353

cognitive 38232

descriptive $2171 \mathrm{f}$

everyday 21 69-71

inappropriate $2168 \mathrm{f}$

prescriptive $2171 \mathrm{f}$

see also semantic-pragmatic categories

PRCAL 32 16ff

discoursal features $3223 \mathrm{f}$

lexico-grammatical features 32

20-23

rhetorical features $3223 \mathrm{f}$

socio-cognitive features $3225 \mathrm{f}$

surface-level features 32 19f

and UNCITRAL Model Law 32

99-114

predicate 17 23-26

complex $3 \mathbf{8} 198$

vs subject 1723

predicative constructions

indirect object in 38 196-199

predicative units $\mathbf{1 2} 204$

minimal 12205

Preisler, Bent

English grammar on functional principles 20 272-277

prepositional phrases $\rightarrow$ PP

prescriptivism 17 113-120

prescriptive pragmatics 21 71f

pressure politics 29 313-324

primary public 1237

prime-notation 1012

Prince, A.

language acquisition 6 46-48

Prior, $\mathrm{P}$.

plagiarism 2842

problem triggers $23157 \mathrm{f}$

process $1780-83,80$ (def)

proclisis 20124

and enclisis

asymmetry between $20128 \mathrm{f}$

opposition between 20 124f

product summary and patient package insert $2565 f$

professionalization $36206 \mathrm{f}$

profiling 35188

prohibition 32106

projection principle $1025 \mathrm{f}$

promises 992

promulgation formula 914

pronesis $\mathbf{3 8} 92$

pronouns 1996

discontinuous groups 17 50-54

in English economics texts 23 34-36

personal

in advertisements 23106 (fig)

Spanish 38 189f

unstressed

Spanish 38190

pronunciation

English 10 125-127, 17 213-234

BBC- 17 217ff, 38183

General British 17224

non-received $\mathbf{1 7} 218$

Oxford accent 17 213ff

received $\mathbf{1 7}$ 213-234, 38 175ff

adoptive RP 17220

anti-RP 17229

boundaries between Cockney

and RP 38181

changes in RP 17 222-224

mainstream RP 17223

near RP 17 220-222

revised RP 17223

wider-based RP 17223

stilted voice 17231

trends in newsreading $17230 \mathrm{f}$

propositional forms $\mathbf{2 8} 128$

proprioception $34204 \mathrm{f}$

prosody 12198

block language 13210

early closure 13210

F0 and prosodic phrasing $13205-$

212

low troughs 13 207-210

Nordic 3 321-325

transcription in speech corpora $\mathbf{1 3}$

$95 \mathrm{f}$ 
prototypology 21229

theory of $\mathbf{2 1} 229$

proverbs

international proverbial sayings

3548

research 35 45-49

psychology

interaction $3269 \mathrm{f}$

public communication 38 7-9, 67

legitimacy $3813 \mathrm{f}$

public services 38 65-81

Danish Public Employment

Service 38 68f

guidance interviews 38 70-73

requests 38 70-73

loss of credibility 3867

and the public 38 66-69

requests in public service encounters 38 65-81

purism $37101 \mathrm{f}$

French 8109

Putnam, L.L.

defining negotiations $\mathbf{1 1} 61$

Pym, Anthony

the concept of locale 36200

localisation 36 197ff

translation 31 206, 36 197ff

\section{Q}

qualifications 3281 (def)

quality 16208 (def)

quality management 16 208-210

and translation theory and practice

16 201-219

Quemada, Bernard

lexicography

bilingual 8 101, 107

computer-aided 8 107f

terminology 1816

questions

closed 32 131-136

multi-turn 32132

open-ended 32 131-136

Quiroz, G.A. translating medical texts 259

Quist, Christian

semantic features in LSP 8 128f

Qvistgaard, J.

dictionaries 8110

\section{$\mathbf{R}$}

raising 16187 (fig), 191

Rasmussen, J.

editing older dictionaries 8107

Rasmussen, Lone Schack

functional grammar 6182

Rastier, François

redefining linguistics $\mathbf{1 6} 10$

Raumolin-Brunberg, $\mathrm{H}$.

The Helsinki Corpus of Early

English Correspondence 13135

reality-based languages 17 84f

Reboul, Anne

text linguistics 16 11f

reciever $\mathbf{1 0} 44$

recontextualization $\mathbf{2 8}$ 90, $\mathbf{3 2} 65$

(def), 72

intertextual 3266

legislative 32 64-71

fuzziness $\mathbf{3 2} 69$

vagueness 3270

Reddy, Michael

conduit model of communication

32239

Redi, Elisabeth M.

spoken and written language 2494

references

in communication 12 29-36

in translation

double role of $\mathbf{1 2} 41$

and interpretation

identification of 12 29-49

non-specific 12 41-46

specific 12 41-46

degrees of freedom 1246 (fig)

reflexives 19 93-111

antipassive 19 83-87

French 19 81-89 


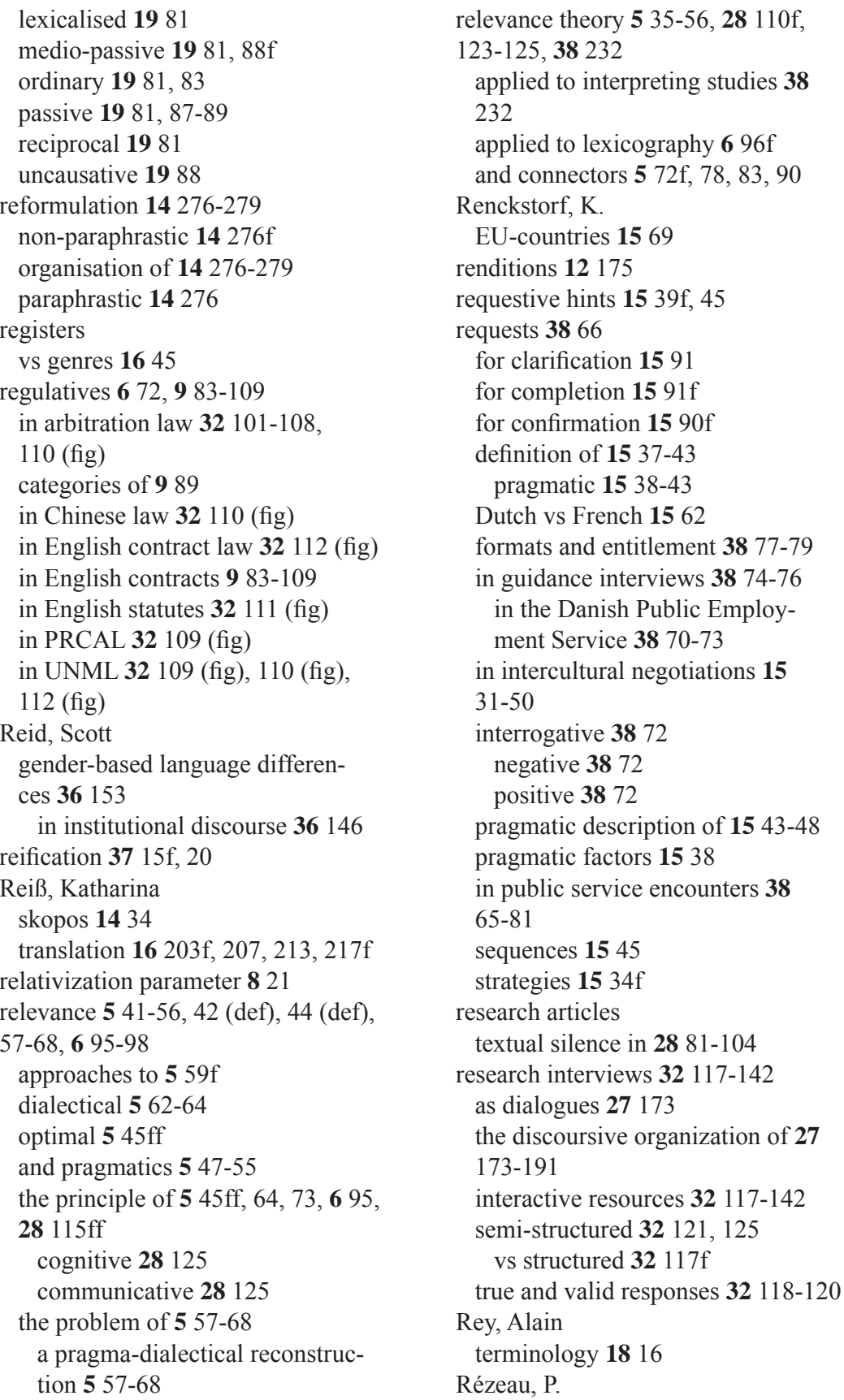


dialectisms and phraseologisms 8 106

rheme 12201 (def)

rhetorics 3748 (def)

business 27 7f, $55-81$

environmental discourse 2759

cultural communication practices

37 55-61

cultural expectations related to

genres 37 47-63

globalizing 37 47-63

of green hotel brochures 27 69-73

verbal strategies 27 70-73

visual strategies 27 69f

history of $\mathbf{2 7} \mathrm{f}$

model for environmental disourse

2758

overview 37 48-50

strategies 19 215, 22 114, 119-131,

3141

passive voice 22 121-131

see also ethos; logos; pathos; persuasion; piestis

rhotic 17116

Riccardi, A.

interpretation research and training 22163

Richards, I.A.

the interaction theory 20167

Richards, K.

plagiarism 2842

Ricoeur, Paul

metaphors in dictionaries 20169

Riel, C.B.M. van

corporate communication $\mathbf{3 8} 12 \mathrm{f}$ (def)

Riggs, F.

descriptive terminology $1823 \mathrm{f}$

Right Node Raising 1010

Riiber, Theis

active-passive theory $\mathbf{3 1} 171$

thesis about four dictionaries per

language pair 6102

Rischel, Jørgen

prosody 3 321-323
Roberts, R.M.

miscommunication 15105

Roberts, Roda P.

interpreting

community 20220

in legal, health and social service

settings 20 211-231

specialized entries in LGP dictio-

naries 37157

Roget's International Thesaurus 20

172

Rognes, Jørn

cross-cultural negotiations $1523 \mathrm{f}$

Rohlfs, G.

cliticization 20134

Rojo, Luisa Martín spoken Spanish 13331

Roloff, M.E.

defining negotiations 1161

Roman, André

language 37158

Romance

negotiation culture 1557

Romance languages 3556

phrases with an infinite verb form

19 93-111

position of clitics 19 93-111

word-order 19 93-111

Romanian

clitics 19 99f

infinitive 19104

Romansh

clitics 19 97f, 101, 110

Romme, A.G.L.

management 2714

Rondeau, Guy

lexicography vs terminology $\mathbf{1 8}$

22-25

root 2514 (def)

Rosbach, Poul

active-passive theory $\mathbf{3 1} 171$

thesis about four dictionaries per

language pair 6102

Rosewarne, David

Estuary English 38175 (def), 175ff 
Ross, J.R.

grammaticality 819

Rossari, Corinne

organisation of reformulation 14

276-279

Roth, Eva

Mikroøkonomisk Glossar 9 207-210

Rothery, J.

genre 16 44f, 53f

Rothkegel, Annely

knowledge 38233

and text types $38224 \mathrm{f}$

Rozas, María V.V.

sentence analysis 6187

RP 17 213-234, 38 175ff

adoptive 17220

anti- 17229

boundaries between Cockney and RP 38181

changes in 17 222-224

mainstream 17223

near 17 220-222

revised 17223

wider-based 17223

Rumelhart, D.E.

past tense simulation 6 43-48

rural subjects 19253 (def)

Russian

grammar 17 75-78

Russo, M.

media and court interpreting 22166

Ryan, H.R.

apologia 3891 (def)

kategoria 3890 (def)

Ryan, Julie

plagiarism 2840

\section{S}

Sackett, G.P.

Lag Sequential Analysis 1512

Sacks, H.

interactions $\mathbf{3 8} 46$

Sager, J.C.

special language 2566 (def) vs general language 2566

Salacuse, J.W.

international negotiations $\mathbf{1 5}$ 10f

Salager-Meyer, F.

academic confrontational discourse 258

Salevsky, H.

interpretation methodology 22164

Sámi 3558

Sammons, Susan

community interpreting $\mathbf{2 0} 221$

Samoyed languages 3557

San José, Valerio Báez

Spanish semantics and syntax 6186

Sánchez, Nora

Accounting Dictionary 38 205ff

Sandemose, Aksel

janteloven (Law of Jante) 27224

Sanders, M.

community interpreter 12128 (def)

Sapir, Edward

drift 38192

Sapir-Whorf hypothesis 38107

structural basis 1997

Sapir-Whorf hypothesis 12 10, 38107 see also linguistic relativity, theory of

Sardinian

clitics 19103

Saussure, Ferdinand de critique against $38108 \mathrm{f}$ language $38107 \mathrm{f}$

la langue 2182

Saville-Troike, M.

communication $15100 \mathrm{f}$

Scandic Hotels 2762

Scandinavia 12215

Scandinavian semi-communication

12216

Scardamalia, Marlene

models of text composition 35190

SCCT 3888

scenes 12 21, 38228 (def), 228f

scenes-and-frames semantics 12 21f, $38228 f$ 
Schaeder, Burkhard

dictionary classification $\mathbf{1 8} 96 \mathrm{f}$

Schäffner, Christina

translatability of metaphors $35184 \mathrm{f}$

Schegloff, E.A.

interactions 3846

Schjoldager, Anne

knowledge systems and translation

38233

manipulation theory $\mathbf{1 2} 16$

names (translation studies) $\mathbf{3 1}$

200-202

semantic networks $38229 f$

simultaneous interpreting

and translation 1412

Schleiermacher, Friedrich D.E.

translation of LSP texts $\mathbf{2 3} 68$

Schlesinger, Miriam

interpreting 12111

community vs court 20215

simultaneous

quality in 22164

translational norms 12 83f, 1466

Schlyter, Suzanne

courtroom language 36133

gender differences in institutional

discourse 36146

Schmid, H.-J.

lexical semantics of begin and

start $13103 \mathrm{f}$

Schmidt, R.

pragmatic information 2185

Schmied, Josef

translation 13169

Schröder, K.

advertising 23 99f

Schubert, Klaus

information vs knowledge 38233

translation $38226 \mathrm{f}$

Schutz, A.

genre 2394

science

the said and unsaid in scientific

discourse 28 81-89

the scientific method $2181 \mathrm{ff}$ scientific research 2 184, 3 287-308, 1268 (def)

unscientific research 1268 (def)

Scollon, R.

plagiarism 2813

Scott, Mary L.

translation skills 2617

screen translation 17 244-250

search structure 34 110-115

Searle, John R

commissives 987

direction of fit 672

equating humans with machines

25117

functionalism 16 179f

indirect speech acts 13332 (def)

purpose of language $\mathbf{1 6} 179$

purpose of linguistic description

16200

regulative acts 984

$\mathrm{SEC} \rightarrow$ Spoken English Corpus

second language $\rightarrow$ L2

second language acquisition

$\rightarrow$ SLA

secondary public 1237

selectional restrictions 8 10f

Seleskovitch, Danica

ESIT 1276

interpreting vs translation 1473

J. Herbert 1277

sense 1044

theory of $\mathbf{1 2} 75, \mathbf{1 4} 32,65$

self-affirmative behaviour 15 124, 126

divergent preferences for $\mathbf{1 5} 116$

Selva, Thierry

lexicography 37156

Selzer, Jack

special topics 3751

semantic triangle 18 51f, 52 (fig)

semantic-pragmatic categories $\mathbf{1 7}$

$278 \mathrm{ff}$

categorizing expressions 17279

(def)

circumstantial expressions 17279

(def) 
evaluative expressions 17279 (def) explicitations 17278 (def)

expressions contained in enumerations 17279 (def)

expressions of contrast to the main theme 17278 (def)

metatextual expressions 17279

(def)

modal expressions 17279 (def)

reformulations 17278 (def)

repetitions 17278 (def)

semantics

in the functional paradigm 6182

of grammatical categories 19

241-251

lexical

corpus studies 29 285-289

linguistic 30181

scenes-and-frames 12 21f, $38228 f$

semantic networks 38 229f

see also domain-level patterns;

semantic-pragmatic categories

semi-experts 18101 (def)

semiotax $28206 f$

semiotic mapping 27 34-40, 36 (fig),

41 (fig)

semiotics

diasemiotic transmissions 17245

isosemiotic transmissions $\mathbf{1 7} 245$

and marketing communication 27

$34 \mathrm{f}$

monosemiotic transmissions 17246

polysemiotic transmissions $\mathbf{1 7} 246$

the semiotic approach 537

Semitic language family $3558 f$

Semprini, Andrea

semiotic mapping $2735 \mathrm{f}$

\section{sender 1044}

sense 5 74, 10 44, 34 62-71

criteria for word sense distinction

34 68-70

empty 13322

theory of 12 75f, 14 32, 65

see also meaning

sentence analysis 17 11-33
English 12 186-189

a pedagogical system 17 11-33

see also syntax

sentence frame

Diderichsen's 12199

Sergo, Laura

Fauconnier/mental spaces 38229

Setton, Robin

interpreting

and communication/negotiation

22165

study of

relevance-theoretic approach $38232 \mathrm{f}$

SHAPE Corpus 7 74f

Sharwood Smith, M.

bilingualism

control and competence 15149

Shaw, Philip

academic writing 288

pragmatics $2169 \mathrm{f}$

Sherman, J.

plagiarism 2840

Shreve, Gregory M.

translation 35190

Sidiropoulou, M.

linguistics, discourse analysis and

interpretation 22165

Sigel, Alexander

abstracting 25127

significant generalization 16185

Silva, Penny M.

South African English 3416

Silva, Raquel

denomination 37159

simulation effect 25 80, 81 (fig)

simulations 34203

complex 34205

simulators 34 203, 206

complex $34205 f$

linguistic 34212

textual $\mathbf{3 4} 212$

simultaneity 17247

singularities 12143

situations 
non-verbal 3719

speech 3742

Skårup, P.

clitics in Old French 20128

enclisis in Old French 20124

Skelton, J.

plagiarism 2842

skopos 12 22, 1434

in simultaneous interpreting $\mathbf{1 4}$

35-38

skopos rule 12 22, 16 206, 214

Skopos Theory 12 22-24, 124, 14

34, 38, 2252

Skoposregel $\rightarrow$ skopos rule

Skyum-Nielsen, Peder

translation model $10116 \mathrm{f}$

SLA 15148

SLA research 15 148f

Slavic

negotiation culture 1557

Slavonic languages $3556 \mathrm{f}$

Sloane Rangers 38179 (def)

Slodzian, Monique

terminology 37152

Smith, J.

plagiarism 2815

Smith, Michael K.

G. Lakoff and M. Johnson 2410

Snell-Hornby, M.

equivalence $1213 \mathrm{f}$

scenes-and-frames semantics $\mathbf{3 8}$

$228 \mathrm{f}$

translation 1270

translation studies $\mathbf{2 1} 225$

word against texts 1218

Snelling, D.

media and court interpreting 22166

social responsibility

model for

developing and implementing 38 56-58

sociolinguistics 13 135-142

accent as social marker 17 216-218

metropolitan sub-variety $\mathbf{1 7} 227 \mathrm{f}$

regional prestige 17 224-226
Stockholm Swedish spoken by

youngsters 9216

urban 9 211-216

see also code-switching; dialects

sociophonetics 9 214f, 38 173-185

sociopragmatic competence 2167

Soelberg, N.

the active/passive principle 8104

Sørensen, Finn

the Adject Theory 38196

modals and modality 31 229-231

topicalization in Danish 12200

Sørensen, Henrik S.

translation $10119 f$

Sørensen, Knud

translation Danish-English 8 85-91

software engineering 36212

Software Quality Assurance 36212

software translation $36211 \mathrm{f}$

Soriano, Fernandéz

clitic-doubled constructions $\mathbf{3 8}$

189 (def)

Soubrier, Jean

English terminology 37158

Spang-Hanssen, Henning linguistic vs encyclopedic know-

ledge 6104

Spanish

clitic-doubled constructions 38

187-202

clitics in 20 126f, 131f

communicative behaviour

vs Danish 3 267-278

vs Scandinavian 3 247-264

cross-cultural negotiations 15 9-27

cultural differences between

Spaniards and Scandinavians 15

115-117

distributive behaviour 3 275-278

grammar

communicative 12 189-192

grammaticalization 38 192-194

indirect object 38 187-202

linguistic change 38 192-194

markedness 38 192-194 
modal auxiliaries 31 231-234

negotiation interaction

vs Danish 3 267-278

negotiations 31 119-133

pull moves

vs Swedish 11 27-46

push moves

vs Swedish 11 27-46

vs Scandinavian

conflicts and consensus 15

111-139

strategic preferences negotiating price 15 130-134

pronouns

personal $38189 \mathrm{f}$

unstressed 38190

standard 784 (def)

verbs 6 185-187

Spar Nord Bank

vs Jyske Bank 27 50f

marketing communication at $\mathbf{2 7}$

47-49

the sales policy of $2747-49$

speaker-based languages $1784 \mathrm{f}$

special language $\rightarrow$ LSP

special language research $\rightarrow$ LSP

research

special topics 37 51-55

culture-based 37 52-61

relationship-related $3758 \mathrm{f}$

specifications $\mathbf{3 4} 70$

Specified Subject Condition $16195 f$

specifiers $\mathbf{1 0} 12 \mathrm{f}$

the specifier rule 1016

speech act theory 948,1537

speech acts 5 62, 9 62f, 3886

in English contract law 6 65-88

impositive 674 (def), 87

indirect 13332 (def), 28109

language of negotiation 15 32-34

legal 6 65-88

macro hybrid 3254

research 15 34-36

silences $\mathbf{2 8} 83$

strategies $1534 \mathrm{f}$ see also apologies; commissives; complaints; declarations; directives; exercitives; promises; regulatives; verdictives

speech corpora

annotating 13 87-98

annotation vs primary data 1388

data

manipulating 13 91-93

organisation and presentation

$1390 \mathrm{f}$

transcription 13 96-98

automatic 1393

contextual phonetic 1396

phonemic 13 93-95

prosodic $1395 \mathrm{f}$

see also COLT; Spoken English

Corpus; text corpora

speech situation 3742

spelling checkers 1063

Spencer-Oatey, $\mathrm{H}$.

business diaculture 2171

Sperber, Dan

code model of communication $\mathbf{2 8}$

114

relevance 5 64, 695

relevance theory $\mathbf{2 8} 115$

Spezifizierung 3471

splitting lemma 12144

Spoken English Corpus

nuclei in the 13 123-133

spoken language

verbalization of writing 2494

and written language 2494

spORDhunde 1312

squinting grammar 1975

stacking 17 26-33

form stack 1727 (def), 27f

function stack 17 27-29, 27 (def)

Stalpers, Judith

rules of conduct 1532

time conception and negotiation

$1564 f$

standard lexical function 7134 (def)

Standard Theory 16196 
Stansfield, Charles W. translation skills 2617

Stark, Mariza

Nuwe Woordeboek sonder grense

38 217-220

Statistische Kollokationsanalyse und Clustering 3459

Steen, Gerard J.

metaphor interpretation 2472

metaphoric processing 2449

stem lemmatisation 34 23-27

step-structure 22 116-119

at micro-level $22118 \mathrm{f}$

Stephenson, G.M.

negotiations 1117

steps

at macro-level 22116

at micro-level 22116

Steyn, Dini

interpreting in legal, health and

social service settings 20 211-231

stipulation rules 32101

Stork, F.C.

problem of equivalence 1211

Strang, B.M.H.

G. Lakoff and M. Johnson 2410

Strangert, Eva

prosody 3324

Strindberg, A.

localisation $\mathbf{1 2} 44$

Strømnes, Frode

successful communication 1231

Strolz, B.

media and court interpreting 22166

Stubbs, Michael

lexical semantics 29 285-289

style 5 53f, 56

casual vs non-casual 9214

cultural context of 23 86-89

legislative 32 55f, 71

in urban speech communities 9

213-216

subholems 38231

subjacency 816

subjacency-violations 8 16-18 subject 1015 (def)

adjuncts $18226 \mathrm{f}$

alternation 38 199f

grammatical

vs explicit 38192

modifiers 18226

early modern English 18 226-228

pleonastic 88

vs predicate 1723

subject predicatives $\mathbf{3 8} 198 \mathrm{f}$

subjects

convergent 19253 (def)

divergent 19253 (def)

rural 19253 (def)

urban 19253 (def)

subjunctives 12190 (def)

substance

vs form 1785

substantives 12190 (def)

subtitling 844 (def), 17 244-250

additive vs non-additive 17247

comprehension 17248

condensation $17247 \mathrm{f}$

reduction 17248

simultaneity 17247

synchrony 17247

vs translation 17245

transmissions

diasemiotic 17245

vs isosemiotic $17245 f$

isosemiotic 17245

monosemiotic 17246

natural vs symbolic 17247

non-synchronous $17246 \mathrm{f}$

polysemiotic 17246

vs monosemiotic 17246

simultaneous $17246 \mathrm{f}$

synchronous $17246 \mathrm{f}$

see also dubbing; interpreting;

machine translation; screen trans-

lation; simultaneity; translation;

voice-over

Suchman, Lucy

interviews 32 118f, 131 
the conversational dilemma 32 120

Sue, Eugène

translation 1238

Sullivan, Dale

specialized knowledge 377

summaries 25 121, 124-127

summarising 844 (def)

Suñer, Avellina

conjunctions 6186

superstructures 621

supportiveness 12 147, 152, 154

strategies

nonsupportive nonassertive 12

151

nonsupportive selfassertive 12151

nonsupportive uninvolvement

12154

supportive involvement 12154

supportive nonassertive $\mathbf{1 2} 152$

supportive selfassertive $\mathbf{1 2} 151 \mathrm{f}$

supportive uninvolvement 12154

Surmiran

clitics in $\mathbf{1 9} 98$

Sursilvan

clitics in 19 97-99, 103

Svane, Brynja

translation 1217

Svanes, Bjørg

Norwegian

as native and foreign language

$1535 f$

Svartvik, Jan

Public Speaking Project in Lund

13123

Svendsen, Lisbet P.

equivalence 8130

information processing strategies

31 10f

Svensén, Bo

defining macrostructure 451

the preface of a dictionary 461

Swales, John M.

academic writing in Western aca-

demia 2543 criticism in English academic

writing 2524

discourse community $\mathbf{2 8} 61$ (def)

genre 16 45f, 48f, 54, 19214 (def)

analysis 27219

categorising 3279

classification 2315

communicative purpose 23 16-18

concept of $2321 \mathrm{f}$

legal discourse 22113

as typified rhetorical action $\mathbf{1 6} 43$

plagiarism 28 51f

silences in communication $\mathbf{2 8} 82$

Swedish

communicative behaviour

vs Spanish 3 247-264

criminal trials

male and female witnesses'

speech 36 129-154

cultural differences between

Spaniards and Scandinavians 15

115-117

Finnish-Swedish community

interpreting 12 109-125

negotiations

pull moves

vs Spanish 11 27-46

push moves

vs Spanish 11 27-46

vs Spanish

conflicts and consensus 15

111-139

strategic preferences negotia-

ting price 15 130-134

Stockholm Swedish spoken by

youngsters 9216

syncategoremata $\mathbf{5} 69$

synonymy 1213

syntactic fallacy 12200

syntactic imperialism 12200

syntax 17 11-33

English 12 186-189

functional 6182

groups $\mathbf{1 7}$ 21f, 29f, 33 
a pedagogical sentence analysis system 17 11-33

sentence analysis 17 11-33

sentence-schema 10 26f

stacking 17 26-31

ungrammatical sentences 8 14-18

X-bar- 8 8, 1718

the essence of 109

the origin and rationale of 10 9-27

synthesis 1769 (def)

$\mathbf{T}$

tacit knowledge 25 113-129

TACITUS 10 118f

Tail-Head Linking 2597

Tanaka, Makiko

simultaneous interpreting $\mathbf{1 4} 136$

Tannen, D.

politeness 1570

TARGET (journal of translation

studies) 1283

Tarp, Sven

learner's dictionaries

amount of lemmata in $\mathbf{3 8} 219$

irregular flexion variants in $\mathbf{3 8} 219$

lexicography vs terminology $\mathbf{1 8} 13 \mathrm{f}$

TAUM METEO 25118

Tavuchis, Nicholas

study of apologies 38 86f

Taya, Norihiko

simultaneous interpreting 14134

Taylor, Christopher interpreting 22 160-167

linguistics, discourse analysis and

interpretation 22165

Taylor, G.

Chinese academic writing $2524 \mathrm{f}$

plagiarism 2814

TCIP 12129

Tebble, $\mathrm{H}$.

intercultural communication, negotiation and interpreting 22165 linguistics, discourse analysis and interpretation 22165
Tebeaux, Elizabeth

business letters

American-Mexican 37 53f

cultural expectations of $\mathbf{3 7} 53$

technical communication 37 9-20,

37-41, 38226

technical language $2370 \mathrm{f}$

technical texts 8 129f, 21227 (def),

$2371 \mathrm{f}$

expressivity in $\mathbf{2 1} 225-231$

prototypical 2366

reading and writing 1566

the technical writer $\mathbf{2 3} 67$

terminology in 2373

translation of 23 65-74

technology $3723 \mathrm{f}$

controversial

mediating 37 23-43

philosophical implications of

introducing $3723 \mathrm{f}$

TEFL Corpus 773

TEI 13 61-64

application of TEI mark-up 13

61-64

temporal iconicity $\mathbf{2 5} 96$

tenor 20167

tense 17 94f, 19 241-251

English vs Danish 7 163-174

future

English vs Danish $7167 \mathrm{f}$

verb tenses in advertisements $\mathbf{2 3}$

105 (fig)

terministic screens 37 17-20, 17 (def)

terminography

legal 22 101-111

new methodology 22 101-111

vs lexicography 18 91-121, 37152

and LSP lexicography 18 91-96,

$119-121$

terminological vs terminographi-

cal definitions 1815

tools 37156

terminology 36 214, 38226 (def) as an academic study in France

$1816 f$ 
adjectives

relational

terminologically relevant 37 $153 \mathrm{f}$

classical

attacks made on 1827

creating terminology from scratch

37153

difference between words and

terms 1823

and documentation $1817 \mathrm{f}$

historical development in French-

speaking countries 18 17-22

and language planning 1819

in France 18 21f

in Québec 18 19-21

legal 22 101-111

new methodology 22 101-111

vs lexicography 18 13-29

and LSP lexicography 18 9f, 13-29

management 36215

modern

the integrated model $1855 \mathrm{f}$

schools 1853

and standardization 1818

in technical texts 2373

and technical translation $\mathbf{1 8} \mathbf{1 8 f}$

term measurement 37 151-159

terminological definitions 37156

vs terminographical 1815

traditional

the objectivist model 18 54f

and Saussurian structuralist

semantics 1853

and socio-cognitive 18 51-88

univocity ideal 18 51-88

terminometry 13 41-43

territories

the concept 2777

ego-centric 2777 (def)

fixed 2777 (def)

situational 2777 (def)

Tesnière, Lucien

dictionaries 6102

Valency Grammar 38196 text corpora 2 179-185, 1310

annotation $\mathbf{1 3} 14$

vs primary data 1388

attested language 29286 (def)

authentic spoken language $783 \mathrm{f}$

at Birmingham University 7 71-79

corpus-based research 7 76-78

the Automatic Abridgement

Project 778

the AVIATOR Project 7 76-78

corpus-based teaching 779

corpus-based translation 13 169-181

Corpus-Bench 13 14-17

of Danish, English and French

contract law 1310

of Danish, German and Spanish

texts on genetic engeneering 1310

of examination papers $775 \mathrm{f}$

intonation structures in $\mathbf{1 3}$ 49-51

in linguistic research 8 127f

for LSP 2527

medium-dependent choice of medium-independent units 13 46-48 medium-dependent units, categories and structures 13 47f

medium-independent units, categories and structures 13 45f and medium-transferability 13

45-52

phonic presentation structure of decoder-encoder 13 51f

phonic presentation structure of encoder 1351

processing $3458 \mathrm{f}$

social representativeness $13140 \mathrm{f}$

specialized 37154

see also Bank of English; Biotec.

ES; Birmingham Corpus; British

National Corpus; COLT; Corpus-

Bench; elexico-corpus;

ENTREVIS; HABLASAL;

Helsinki Corpus of Early English

Correspondence; JUR-korpus;

LOB-Corpus; London-Lund

Corpus; Longman/Lancaster 
Corpus; New Corpus of Spoken English; PE77; SHAPE Corpus; speech corpora; Spoken English Corpus; TEFL Corpus text linguistics 16 9-13

Centre for Text Linguistics (in Denmark) 16 9f

text summarisation 25 113-129

abstracts 25 121, 125-127

conference 28 95-99

and textual silence 28 95-99

extracts 25121

summaries 25 121, 124-127

text-external matrix 27220 (fig)

text-internal matrix 27220 (fig)

textogram 27216 (def)

texts $\mathbf{1 0}$ 41, 12 24f, $3 \mathbf{8} 224$ (def)

accessibility $\mathbf{8} 46$

analysis 5 60f

argumentative 5 61f

chunks 6 21f

classification 23 13-23

comprehensibility of 37 67-89

condensation 25 113-129

programs $25113 \mathrm{f}$

controlled 32190

conventions 32 171f, 174, 175 (fig)

cultural constellations $38230 \mathrm{f}$

difference between British and

American editions 32 186ff

function 1222

intratextuality 3265

knowledge in $\mathbf{3 8} 224 \mathrm{f}$

LSP

economics

English 23 25-40

external variables $2336 \mathrm{f}$

internal variables 23 28-36

focal terminology 23 32-34

lexical aspects 23 29-32

legal

pronouns in 23 34-36

comprehensibility of 36 15-39

German

court decisions 36 15-39 linguistic methods in the description of $2299 \mathrm{f}$

level of specialization 37154

lexical inventory 37116

medical 25 63-73

translation of 25 49-59

optimizing 37 115-126

readability of

English vs German 37 116ff

reformulation phenomena 37154

technical 8 129f, 21227 (def),

$2371 \mathrm{f}$

expressivity in 21 225-231

prototypical 2366

the technical writer 2367

terminology in $\mathbf{2 3} 73$

translation of 23 65-74

translating 37 115-126

the macrostructure of 27224

non-instructive

comprehensibility of 37 67-69

organisation 2579 (def), 81-86, 90

problem of originality 32 185-198

the vanishing original 32 185-198

research

content-oriented 26 40, 47-53

form-oriented 26 40, 57-61

process-oriented 26 40, 53-57

superstructures 32 169, 172, 180,

182

and translation 26 11-13

types $620 \mathrm{f}$

and knowledge $38224 \mathrm{f}$

in translation 12 46-49

typology 23 13-23

translation-oriented 2368,74

written vs spoken 846

textual mapping 32 62-64, 62 (def)

textual silence 2883 (def)

TG-grammar 10 11-15, 19

Thagard, Paul

connectionism $25128 \mathrm{f}$

theme 12201 (def), 21201

Theory of Binding and Control 16196 
thesauri

vs dictionaries 20 169-178

think-aloud protocols 625

Thom, René

catastrophe theory 12143

elementary catastrophes $\mathbf{1 2} 144$

Thomas, J.

miscommunication 15105

Thome, Gisela

Fauconnier/mental spaces 38229

Thompson, Kristin

dimensions of film editing 34207

Thompson, Sandra

linguistic change 38190

Thomsen, Christa

CSR communication 388

Thorsen, Nina Grønnum

$\rightarrow$ Grønnum, Nina

Thrane, Torben

knowledge and simultaneous

interpreting $\mathbf{3 8} 223$

three-party two-language model 1044

Tijus, A.

interpreting 22162

time

the concept 15 63-66

Anglo-Germanic and Latin

(=Hispanic/French etc.) 15 53-76

Tirkkonen-Condit, S.

interpretation methodology 22164

translation $\mathbf{3 1} 206$

Tobler-Mussafia law 20 126, 132

Toft, Bertha

Mikroøkonomisk Glossar 9 207-210

Togeby, Ole

functional grammar 6182

Tominaga, Masayuki

simultaneous interpreting 14133

Tommola, Jorma

errors and omissions in interpre-

tations 2253

interpretation research policy 22 $162 \mathrm{f}$

tone groups $\rightarrow$ tone units

tone units 13 50, 58, 126-131 top-down processing 1225

topicalization 8 19f

topics

special 37 51-55

culture-based 37 52-61

relationship-related $3758 \mathrm{f}$

topline $\mathbf{1 3} 205$

topological analysis $\mathbf{1 2} 200,202$

topological fallacy 12202

topological parser 3620

topological structure 12199

Topping, Suzanne

translation 36202

Torikai, Kumiko

intercultural miscommunication

14140

Torsello, C.T.

linguistics, discourse analysis and interpretation $22165 f$

Toulmin, Stephen

casuistry $\mathbf{3 8} 92$

tourist brochures

localisation 32193 (fig)

Tournay, J.

legal text corpora 8128

translation 8110

Toury, Gideon

The Manipulation School 1270

translation 12 71f, 2669

the concept of $\mathbf{1 2} 68$

normativity 12172

norms 1272 (def), 73, 14 67f

relationships $1273 \mathrm{f}$

Trace Theory 16 195f

Tracy, Karen

the relevance problem 557

tranformations

root 819

structure-preserving 819

transcreation 36210

transcription

spoken data 13 96-98

transformational rules 16188

transgenic wheat 37 23-43

translation 845 (def), 1044 (def), 
1211 (def), 12 (def), 16203 (def), 20 165-179, 26 11-13, 15-35, 31 47-64, 32 185-198, 36 197ff accuracy 2617

Bible- 12 11, 35 287-290

and cognitive structures 13 169-181

and communication 23 77-91

as communication 12 36-41

company financial data (Danish-

English) 32 145-164

computer assisted 6 15-29

textpatterns in 6 16ff

Comtec Translations 16 210-213,

219

corpus-based 13 169-181

criticism 12 67f

as cross-cultural-socio-historical-

pragmatic transfer 1262

cultural constellations $\mathbf{3 8} 230 \mathrm{f}$

cultural transfer $\mathbf{3 8} 222$

culture-bound expressions $1251 \mathrm{ff}$

Danish-English 8 85-91

degrees of freedom 12 46f

documentary $1223 \mathrm{f}$

documentation $36212 \mathrm{f}$

dominance of linguistics 1283

drafting principles

recent developments in 9 134-139

into English 32 147f

equally authoritative 32 195-198

errors 26 19f

EU 32 195-198, 34 293-297

evaluation of 26 15-35

expression 2617

financial reporting 32 145-164

French-English 2670

homo transferens 1269

humour 14 44-48

instrumental $1223 \mathrm{f}$

inter-generic $2565 \mathrm{f}$

interdisciplinarity 12 16-20, $1788 \mathrm{f}$

and interpreting 8 43-53, $1043 \mathrm{f}$

vs interpreting 8 43-53, 10 43f,

14 31-50, 31ff, $72 \mathrm{f}$

research product-oriented $1431-50$

theoretical interface 14 31-50

ISO certifications 16 209-212

and knowledge systems 38 221-234

knowledge-related aspects of $\mathbf{3 8}$

221-234

for language purposes 30 199-211

linguistic theory of $\mathbf{1 4} 32$

literal 1211

vs technical $2368 \mathrm{f}$

vs localisation 36210 (fig)

LSP 6 106, 10 115-123, 12 19f,

23 65-74, 37 115-126

brewery brochures 27226

equivalence $1214 \mathrm{f}$

legal

courses for translators $\mathbf{2 2} 131 \mathrm{f}$

the legal translator 9 113-139

textual knowledge in 32 167-

183

medical texts 25 49-59

metaphors 24 57-79

technical 21 225-231, 23 65-74

vs literary $2368 \mathrm{f}$

the state of 23 67-69

and terminology $1818 \mathrm{f}$

machine

knowledge representation in $\mathbf{1 0}$

118, $38223 f$

knowledge-based 38224

meaning identification 619 (fig)

metaphors 35 183-205

in LSP texts 24 57-79

models $10116 f$

Bialystok 31 52f, 53 (fig)

CBS 31 53-55, 54 (fig)

Triangle 31 51f, 52 (fig)

normativity 12 68f, 172

novels

actions scenes 34 199-221

Online Help 36212

ontology-driven translation

management $\mathbf{3 8} 225 \mathrm{f}$

oral

modal expressions $3156 \mathrm{f}$ 
vs written 1474

and picture verbalization 30 199-

211

practice of 16 201-219

pragmatic aspects of 31 197-207

problems of (social) gender 12

51-62

process 32159 (fig), 159f

quality assessment 26 15-35

and quality management 16 201-

219

recipient roles in 23 203-219

recycling 32 194f

references

double role of $\mathbf{1 2} 41$

identification 12 29-49

specific and non-specific 12 41-46

screen- 17 244-250

sentence-for-sentence $1787 \mathrm{f}$

simple vs complex scenarios 32

185-187

skills 2617

software $36211 \mathrm{f}$

source language 6 16ff

subbranch of applied linguistics?

$1270 f$

target language $616 \mathrm{ff}$

teaching of 16 201-219

technical $38226 \mathrm{f}$

technology 36213

text types in 12 46-49

three laws of $\mathbf{1 6} 202$

three rules governing $\mathbf{1 6} 206,214 \mathrm{f}$

tools 32 161-163

traditional views on 12 10-16

the translation process

expertise and explicitation in $\mathbf{3 8}$

235-238

translational norms 12 83-85, 14

114-116

concept of 12 72-74, 14 66-69

initial norms 1467

operational norms 1467

preliminary norms 1467

translational relationships $\mathbf{1 2} \mathbf{7 3 f}$ theoretical model 14 81-83

the translator 1240

behaviour and language usage

26 65-78

qualities 32160

Übersetzungswissenschaft 12 69-71,

1473

untranslatability 14 80-84

value chain of $\mathbf{1 6} 212$ (fig)

word-for-word $1787 \mathrm{f}$

see also adequacy; dubbing; equivalence; Fidelitätsregel; interpreting; Kohärenzregel; simultanei-

ty; skopos rule; subtitling; voiceover

translation research 12 71, 167-170, 26 97-115

for students 26 97-115

theory-related 26 81-94

tradition 12 168-170

usefulness of 26 98-102

translation strategies 12 42, 73 (def), 32159 (fig), 159f

commented and explained reproduction 12 42-44

direct reproduction 12 42f

generalisation 12 42, 45-47

localisation 12 44f, 47f

micro- vs macro- 2457

translation studies 12 9-25, 70

(def), 171, 31 197-207, 38 221f

branch of applied linguistics? 12

168

definitions 31 197-207

descriptive 12 66-69

names 31 197-207

research tradition 12 168-170

Translationswissenschaft 1473

translation theory 12 9f, 69, 16

201-219

history of 1269

linguistic-oriented

rebellion against 1277

schools 12 70f, 14 21f, 16 203-207 
school of functionalism 14 31ff, 16 205-207

Translationswissenschaft 1473

translatology 12 11, 69-71

efforts 1279

two schools $1270 \mathrm{f}$

Translog 35 190, 37 68f, 72-74

transversality 24 81-105

Traugott, E.C.

grammaticalization 38191

markedness 38190

triangulation $38227 \mathrm{f}$

TRICS 6 46, 48

Trier, Jost

structuralist field theory 21228

trigraphs $13187 \mathrm{ff}$

trilogues 1236

Trosborg, Anna

complaints 2172

cross-cultural negotiations $\mathbf{1 5} 15 \mathrm{f}$

Danish learners of English 1536

international arbitration 3211

simulations vs real-life situations

1524

Trudgill, Peter

Estuary English 38183

standard English 17218

turn-taking 3 258-260, 13 66, 3845

Turner, Mark

conceptual blending theory $\mathbf{2 4} 138 \mathrm{f}$

conceptual integration 24140

Great Chain of Being 30184

Twin Cities Interpreter Project $\rightarrow$ TCIP

Two-Level Hypothesis 1013

Tytler, Alexander F.

three laws of translation 16202

$\mathbf{U}$

Uckmar, M.

media and court interpreting 22166

Übersetzungswissenschaft 12 69-71,

1473

Ugric languages 3557
Ulijn, Jan M.

autobiographical note 1576

Dutch and French cultural beha-

viour 15 60f

rhetorical expectations 3762

UNCITRAL Model Law 32 17ff,

77-97

vs Brazil's Arbitration Law 32

45-72

compared with PRCAL 32 99-114

discoursal features $3223 \mathrm{f}$

lexico-grammatical features 32

20-23

rhetorical features $3223 \mathrm{f}$

socio-cognitive features $3225 f$

surface-level features 32 19f

understanding

assymetrical 1236 (fig)

mechanisms of 1234 (fig)

uniformitarianism 13136

universals

implicational 821

UNML $\rightarrow$ UNCITRAL Model Law

upgraders 1546

Upper-crust 17 226-228

Uralic language family $3557 \mathrm{f}$

urban subjects 19253 (def)

utterance interpretation 28 109-132

utterances 5 74ff, 15 134, 36137 (def)

considered as topological

domains 12199

constative 948 (def), 56

distance

maximum 15 134-136

medium 15 134, 136

minimum 15 134, 136

ironical $552 \mathrm{f}$

metaphorical 552

nonsupportive involvement 12154

nonsupportive negative 12153

nonsupportive positive $\mathbf{1 2} 153$

performative 948 (def), $48 \mathrm{f}$

explicitly 949

primary 949 
supportive positive 12154 utterance range $\mathbf{1 3} 205$

\section{V}

valency grammar 38 196f

Van Deth

denying translation 2550

Vance, Kathleen

grammar course for engineering technology 31 11f

Vandermeeren, $\mathrm{S}$.

business communication 31 9f

VARBRUL

modal auxiliary verbs 13 71-84

Varner, Iris

communication expectations $\mathbf{3 7} 62$

Vasquez-Ayora, G.

denying translation 2550

vehicle 20167

Veiga, Alexandre

Spanish verbs 6 185-187

verbal gender 17 93-95

verbs

arguments 38197

fundamental $\mathbf{3 8} 198$

aspectual

begin vs start

in British, American and Indi-

an English 13 99-121

in Brown Corpus, LOB-

Corpus and Kolhapur 13 105-

120

lexical semantics of $\mathbf{1 3} 103 \mathrm{f}$

surface grammar of $\mathbf{1 3}$ 100f

syntax and semantics of $\mathbf{1 3}$ 100-105

auxiliaries 13 71-84, 19 101f

English vs Danish 7 164f

modal 19106

Spanish 31 231-234

as axiological words $\mathbf{3 0} 183$

categories and their internal struc-

ture 17 93-95

causative 19 106f cirteria for a hierarchy 20 122-124

of communication 1984

complex 1794

discontinuous groups 17 49f

the grammar of seem 16 177-200

infinite

in Romance languages 19 93-111

internal structure of VPs 10 19f

modal 31 229-231

implicitly performative 9 48-50

with multiple functions $993 \mathrm{f}$

with a sound resemblance

Danish-English 31 57-61

frequency 31 57-60 (fig)

of motion $\mathbf{1 9} 86$

nominalization of verbal struc-

tures $628 \mathrm{f}$

performative 6 71, 9 14, 48-50

psychological 19 84f

reflexive

French

antipassive in 19 75-90

functional description of $\mathbf{1 9}$

$75-90$

simplex 1794

Spanish 6 185-187

tenses

in advertisements 23105 (fig)

uncausative 1988

Zulu $3421 \mathrm{f}$

verdictives 6 72, $913 \mathrm{f}$

Verlinde, Serge

lexicography 37156

Vermeer, Hans J.

independence of translation stu-

dies 2669

paraculture 1440

skopos 1434

translation 16 205-207, $216 \mathrm{f}$

three rules governing 16 206, 214f

Vestergaard, $\mathrm{T}$.

advertising 23 99f

Vet, Co

text linguistics 1612 
Viaggio, $\mathrm{S}$

interpretation research and training 22163

viewpoints 5 75-77

Vik-Tuovinen, G.-V.

simultaneous interpreting 1412

Vikner, Carl

noun phrases in legal language 8 $130 \mathrm{f}$

text corpora in linguistic research

$8127 \mathrm{f}$

Vikør, Lars S.

Nordic languages 12 215-217

visual blends 24 131-158

visual images 24 138-144

voice alternations 19 76-79

voice-over 17246

Volgaic languages 3557

VP

the internal structure $1019 \mathrm{f}$

Vuorikoski, A.

linguistics, discourse analysis and interpretation 22165

\section{W}

Wackernagel law 20 118, 125

Waddell, Craig

genetic engineering 3725

Waddington, Christopher

text and translation 26 11f

Wade, Rodrik

Black South African English 3416

Wadensjö, C.

dialogue interpreting 1412

in Sweden 32238

the interpreter's role 20225

interpreting as interaction 12 167181

media and court interpreting 22166

Wagner, Emma

translating for EU 34 293-297

Wagner, Johannes

negotiations 3201
Walker, Esther

negotiations 1110

Walzer, Arthur E.

special topics 3752

Wande, Erling

The Skopos Theory 1224

successful communication 1231

Wandruszka, Mario

gender problems in translation 12

55,61

Web Course Tools $\rightarrow$ WebCT

web resources

vs LSP dictionaries 37153

WebCT 31 83-100

development 31 86-88

features 31 88-91

Wegener, Helle

nominalizations in Danish 8131

Weinbach, L.

complaints 2172

Weinrich, Harald

categorisation of metaphors $\mathbf{2 4} 70$

metaphors 2460 (def)

Textpartitur 27218

Weiss, Steven E.

culture-based special topics 3754

Weizman, Elda

requestive hints $\mathbf{1 5} 40,45$

Wells, J.C.

mainstream RP 17223

Welsch, Wolfgang transversality 24 100-103

Wenger, E.

communities of practise 37 14, 20

model of meaning $3715 \mathrm{fig}$

technical communication 3716

West, Candance

courtroom language 36133

Western individualism 15 55f

Wh-Island Constraint 816

Wh-movement 8 16f

Wh-trace 818

Whalley, P.

hypertext 17 63f

Whittemore, G. 
blurred genres 2395

Whorf, Benjamin L.

Sapir-Whorf hypothesis 38107

Wickelfeatures 6 43f, 46

Wickelphones 644

Widdowson, H.G.

in-groupness 2878

Wiegand, H.E.

criticising Wolfgang Mentrup 31

177

dictionaries

classification of $\mathbf{6}$ 92, 31 178-185

as texts $\mathbf{6} 95$

users 34120

lexicographic functions $31171 \mathrm{ff}$

linguistic vs encyclopedic know-

ledge 699

Wijst, P. van der

politeness strategies 15 61f

Williams, S.

skill components in simultaneous

interpreting 22164

Williamson, Oliver F.

negotiations 31123

Wilson, Deirdre

code model of communication $\mathbf{2 8}$

114

relevance 5 64, 695

relevance theory $\mathbf{2 8} 115$

Wilss, Wolfram

translation 2557

development in 2550

Windfeld, Stine

communication policies 38 19ff

Wingate, Ursula

learner dictionaries $30215 \mathrm{ff}$

Winsor, Dorothy

communication expectations 3762

Wiseman, R.L.

Latin (=Hispanic/French etc.) vs

English culture 1558

Wittgenstein, L.

criticism of field theory 21228

Woods, Nicola

courtroom language 36133
Wooffitt, Robin

interview data 27 178-180

semi-structured interviews 32121

Woolever, Kristin R.

cultural communication practices 3756

Word Finder 7130

word order 12 198-206

Danish 12199

Romance languages 19 93-111

word watchers 1312

words

against texts 1218

vs in isolation 1218

axiological 30183

complex 2515

emotional 30183

simple 2515

termhood of 13 41-43

Words and Phrases Legally Defined

macrostructure $454 \mathrm{ff}$

words-to-world 6 72, 984

World Wide Web 24 88-105, 36213

see also Internet

world-to-words 6 73, 984

Wotjak, Bárbara

verbs 6187

Wotjak, Gerd

Spanish verbs 6 185-187

writing $2492 \mathrm{f}$

academic 25 23-44

criticism in 25 23-44

Chinese 25 24f

Czech 2525

English 2524

German 2525

Malaysian 2525

Polish 2525

plagiarism 28 7, 13 (def), 39-56

learners of English 28 11-31

reasons 2812

writing of Japanese university

students 28 16-31

in Western academia 2543

legal 32 45-72 
syntactic discontinuities 3281 (def)

textual organization 32 51-54 variation in $\mathbf{3 2} 49$ (fig) generic 32 45-72

pictorialization of 24 95-100

scriptualization of language 2494 spoken and written language 2494 technical $38226 \mathrm{f}$

verbalization of 2494

Wüster, Eugen

CTN 1815

dreiteiliges Wortmodell 1852

(fig)

Wyk, Egidius B. van verbs in Zulu 34 21f

Wyld, H.C.

regional dialects 17214

Wynn, Rolf

doctor-patient communication 23 250-254

\section{X}

X-bar syntax 8 8, 1718

the essence of $\mathbf{1 0} 9$

the origin and rationale of 10 9-27

X-bar-theory 16196

Xing, J.

pragmatics of the business diaculture 2171

\section{$\mathbf{Y}$}

Yates, J.A.

genres 2395

Yli-Jokipii, Hilkka

book information and place

description 3111

Yoshitomi, Asako

interpreting 14135

\section{$\mathbf{Z}$}

Zaixi, Tan

translation 2550

Zalka, I.

intercultural communication,

negotiation and interpreting 22165

zero-sum games $\mathbf{3} 268$

Zethsen, Karen K.

medical texts 258

technical texts 2310

Zettersten, Arne

New Oxford English Dictionary

$12214 f$

Zoëga, Anne

translation

vs interpreting 1472

and simultaneous interpreting

$1475 f$

Zulu

verbs 34 21f

Zwicky, A.M

simple vs special clitics 20117 
